\title{
Making memory strategic : studies on the cognitive and biological basis of memory strategies in children and adults
}

Citation for published version (APA):

Schleepen, T. M. J. (2014). Making memory strategic : studies on the cognitive and biological basis of memory strategies in children and adults. [Doctoral Thesis, Maastricht University]. Datawyse / Universitaire Pers Maastricht. https://doi.org/10.26481/dis.20140424ts

Document status and date:

Published: 01/01/2014

DOI:

$10.26481 /$ dis.20140424ts

Document Version:

Publisher's PDF, also known as Version of record

Please check the document version of this publication:

- A submitted manuscript is the version of the article upon submission and before peer-review. There can be important differences between the submitted version and the official published version of record.

People interested in the research are advised to contact the author for the final version of the publication, or visit the DOI to the publisher's website.

- The final author version and the galley proof are versions of the publication after peer review.

- The final published version features the final layout of the paper including the volume, issue and page numbers.

Link to publication

\footnotetext{
General rights rights.

- You may freely distribute the URL identifying the publication in the public portal. please follow below link for the End User Agreement:

www.umlib.nl/taverne-license

Take down policy

If you believe that this document breaches copyright please contact us at:

repository@maastrichtuniversity.nl

providing details and we will investigate your claim.
}

Copyright and moral rights for the publications made accessible in the public portal are retained by the authors and/or other copyright owners and it is a condition of accessing publications that users recognise and abide by the legal requirements associated with these

- Users may download and print one copy of any publication from the public portal for the purpose of private study or research.

- You may not further distribute the material or use it for any profit-making activity or commercial gain

If the publication is distributed under the terms of Article $25 \mathrm{fa}$ of the Dutch Copyright Act, indicated by the "Taverne" license above, 


\section{Making memory strategic}

Studies on the cognitive and biological basis of memory strategies in children and adults 
Copyright@ Tamara Schleepen, Maastricht 2014

Production: Datawyse / Universitaire Pers Maastricht

ISBN 9789461593207

Financial support for the publication of this thesis was kindly provided by Uitgeverij Schoolsupport (www.schoolsupport.nl) and by pedagogue and coach Ingeborg Dijkstra-Verbeek (www.steppingstonescoaching.nl) 


\section{Making memory strategic}

\section{Studies on the cognitive and biological basis of memory strategies in children and adults}

\section{PROEFSCHRIFT}

ter verkrijging van de graad van doctor aan de Universiteit Maastricht, op gezag van de Rector Magnificus, Prof. dr. L.L.G Soete, volgens het besluit van het College van Decanen, in het openbaar te verdedigen op donderdag 24 april 2014 om 14:00 uur

door

\section{Tamara Maria Johanna Schleepen}

geboren op 23 oktokober 1983

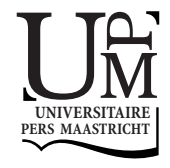




\section{Promotor}

Prof. dr. Bernadette Jansma

\section{Copromotor}

Dr. Lisa Jonkman

\section{Beoordelingscommissie}

Prof. dr. Peter de Weerd (voorzitter)

Dr. Petra Hurks

Prof. dr. Peter Muris

Dr. Henry Otgaar

Prof. dr. Maartje Raijmakers (Universiteit Amsterdam) 


\section{Contents}

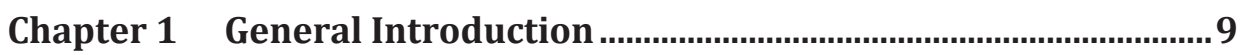

1.1 What is known about the typical development of short-term- and

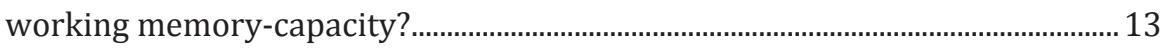

1.2 Most distinguished strategies in the developmental literature ............................ 15

1.3 How are the different memory systems involved in the rehearsal and

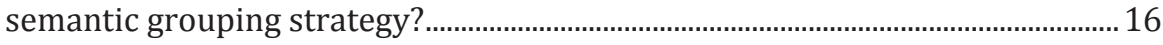

1.4 Evidence for a possible role of WM in the semantic grouping strategy ..............18

1.4.1 Evidence for a mediating role of working memory capacity in the effective application of semantic grouping strategies in adults ..............18

1.4.2. Support from developmental research for a link between WMC and semantic grouping strategy use in childhood ....................................... 18

1.5 Why use ERPs to study the neural basis of elaborative memory

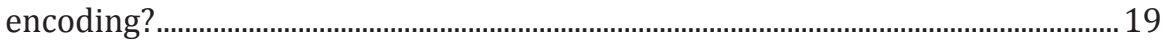

1.6 The neural basis of elaborative encoding, in specific of semantic grouping .... 20

1.7 Aims and outline of the present thesis......................................................................... 24

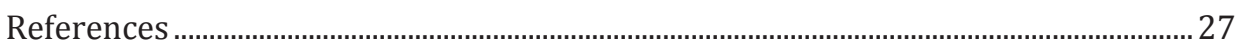

\section{Chapter 2 The development of non-spatial working memory} capacity during childhood and adolescence and the role of interference control; an N-Back task study............ 31

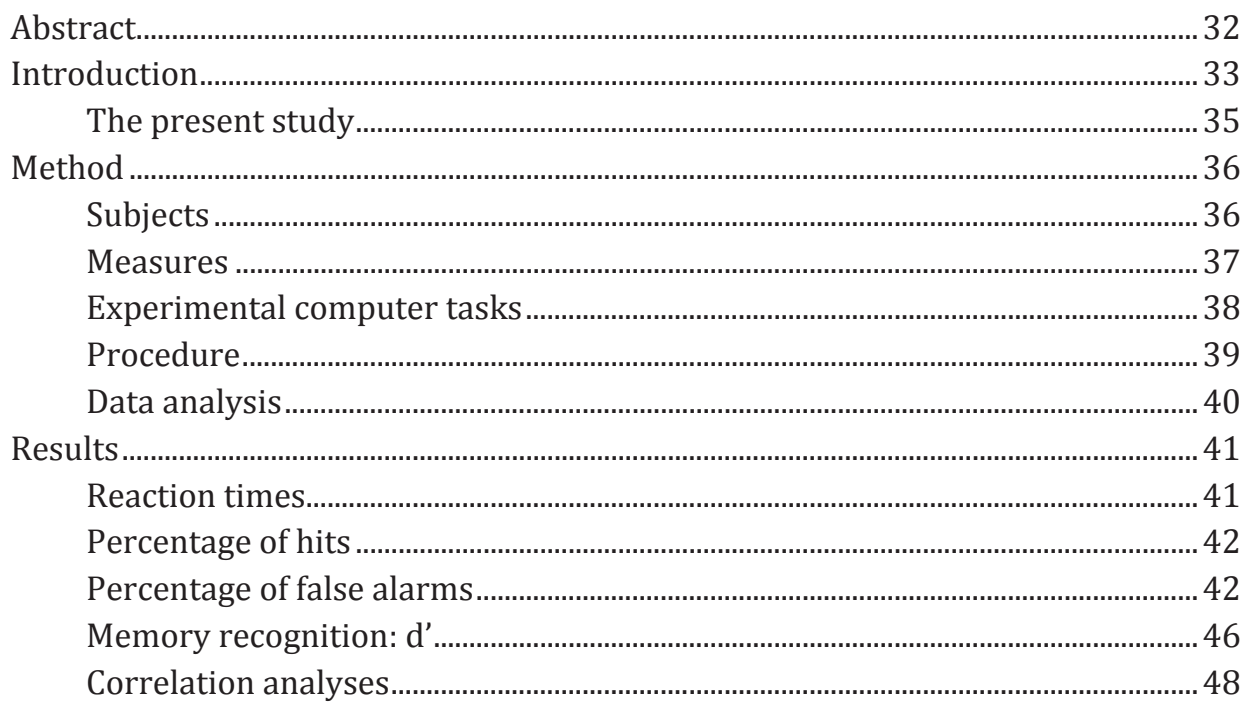


Discussion.

The involvement of interference control and manipulation in the N-Back task

The development of WM maintenance during low or high distraction ............. 49

Limitations of the present study ............................................................................... 53

References

Chapter 3 Children's use of semantic organizational strategies is mediated by working memory capacity ........................... 57

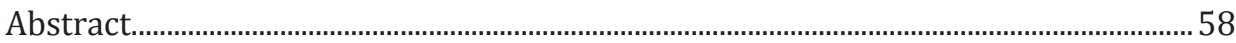

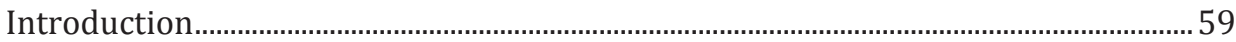

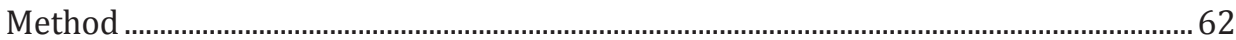

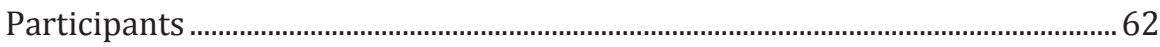

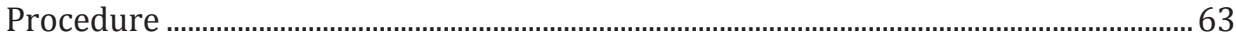

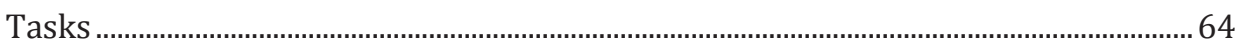

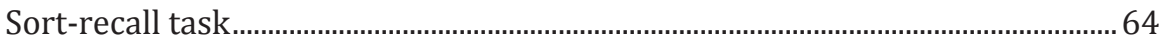

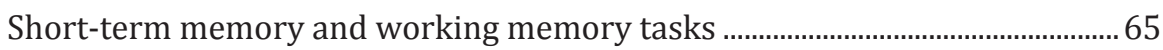

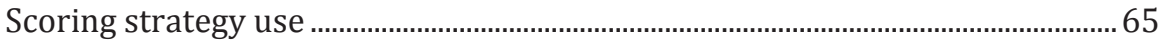

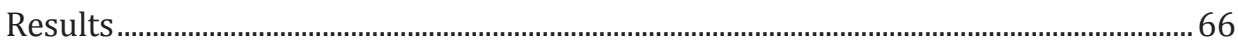

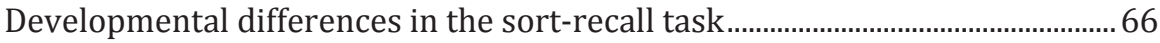

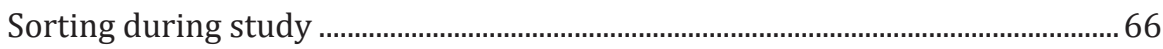

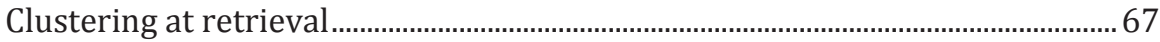

Recall 67

Relations among age, strategy use and recall ......................................................... 68

The role of STM/WM in successfully using semantic grouping strategies .......68

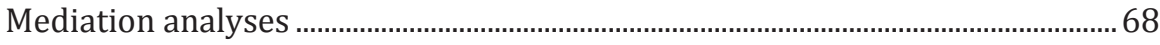

Differences in STM/WMC between strategy and non-strategy users ................. 70

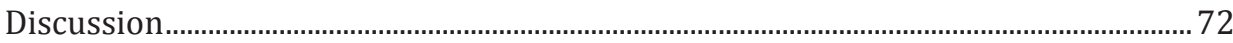

Developmental differences in the use of semantic grouping strategies ............ 72

Roles of short-term and working memory in children's effective semantic

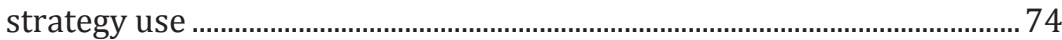

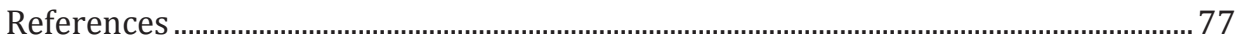

Chapter 4 A longitudinal study of semantic grouping strategy use in 6-12 year-old children: investigating developmental phases, strategy transfer and the role of working memory ............................................................... 81

Abstract. 


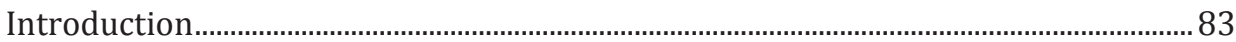

Development of semantic grouping strategies............................................................. 83

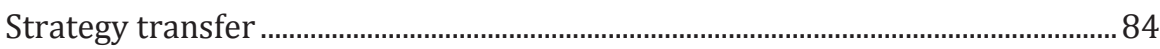

Role of WMC in semantic grouping strategy use..................................................... 85

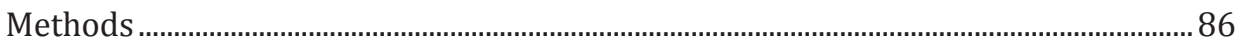

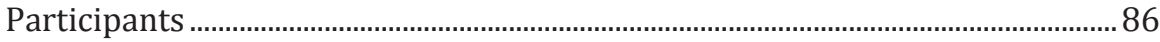

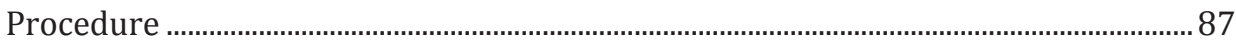

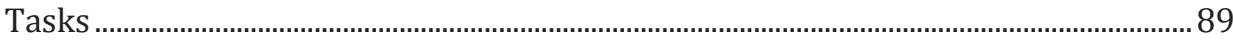

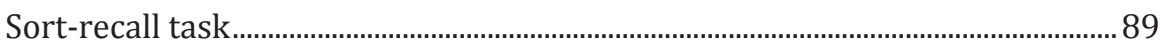

Transfer sort-recall task........................................................................................... 90

Short-term memory and working memory tasks .................................................. 90

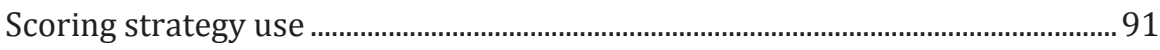

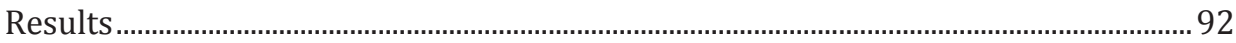

Longitudinal development of semantic grouping strategies ............................... 92

Relation between strategy use and recall ................................................................ 93

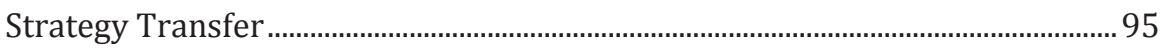

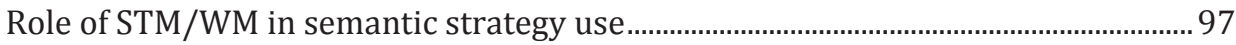



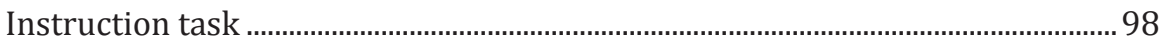

Discussion........................................................................................................................ 98

Longitudinal development of semantic grouping strategies .............................. 99

Strategy transfer ..................................................................................................... 100

Role of WMC in semantic grouping strategy use.................................................. 102

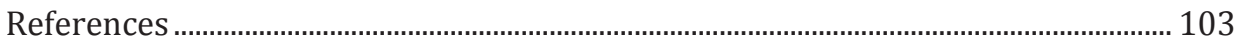

\section{Chapter 5 Dissociating the effects of semantic grouping and rehearsal strategies on Event-Related Brain Potentials .................................................................................... 105}

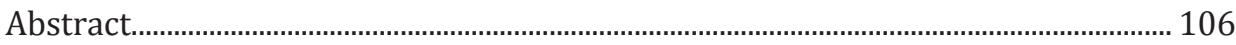

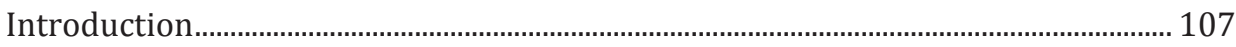

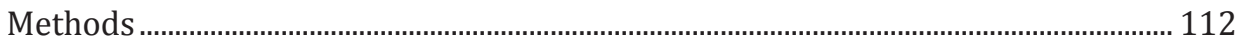

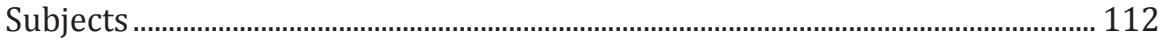

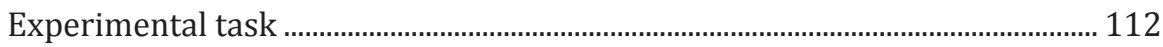

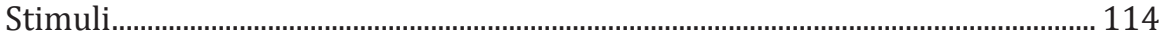

Electrophysiological Recording and Analysis.................................................... 114

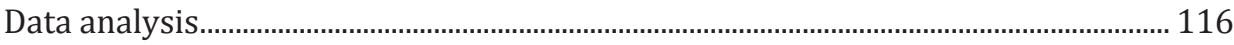

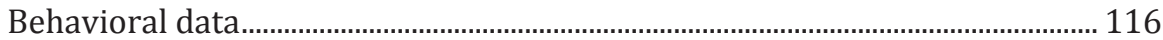

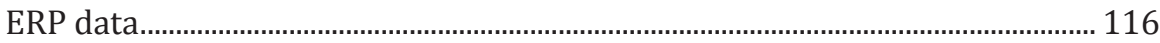

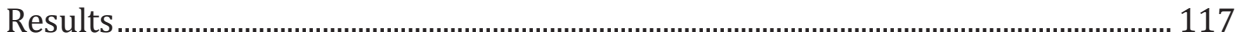

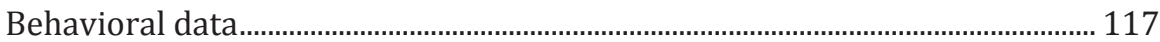




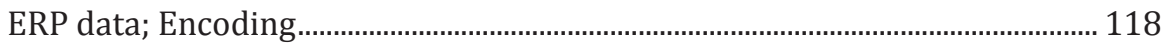

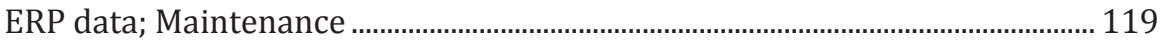

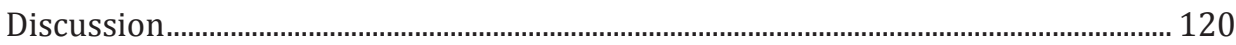

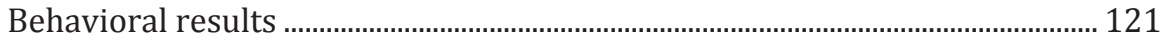

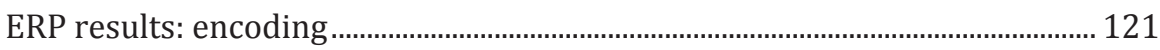

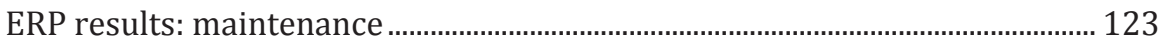

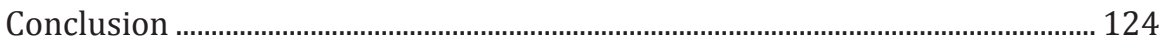

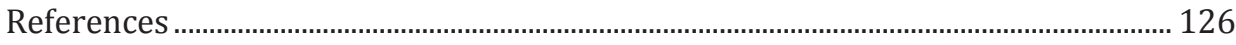

Chapter 6 Summary and Discussion .....................................................129

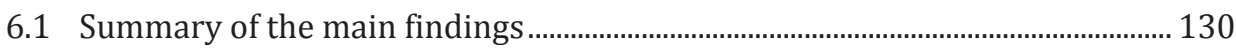

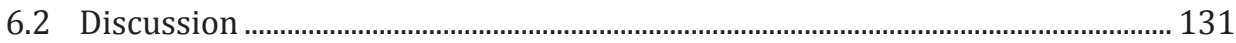

6.2.1 The role of executive control in the delayed development of WMC.... 131

6.2.2 The importance of WMC in using the semantic grouping strategy during childhood ........................................................................................... 132

6.2.3 How might the observed neural mechanisms (chapter 5) be linked to the developmental pattern of the semantic grouping strategy

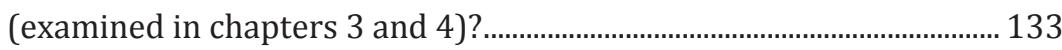

6.2.4 Conclusion and practical implications.......................................................... 136

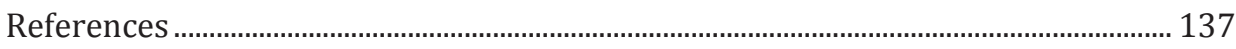

Dankwoord .............................................................................................139

Curriculum Vitae ............................................................................. 143 
Chapter 1

General Introduction 
Learning and memory are intrinsically related to each other. For learning to be successful we must store information in memory permanently; similarly, if we want to store new information in memory this is easier if we can relate it to information learned at an earlier moment. Whereas memory is important for all of us, there is no period in life in which demands on memory are greater than in the school years.

Most cognitive- psychological theories define memory as a system that comprises several different memory stores; these are sensory memory, short-term memory (STM), working memory (WM) and long-term memory (LTM). Also at the neurobiological level these different memory systems have been distinguished and have been shown to rely on (partly) different brain networks (e.g. Smith \& Jonides, 1999). Although all memory systems interact with each other during performance of cognitive activities, they serve different functions and contribute in their own way to learning (Gathercole \& Alloway, 2008).

Sensory memory picks up information provided by the different senses and retains it for a short period of time until it is replaced by new sensory information. If we want to use the information in sensory memory and process it further to short-term memory (STM), we must pay attention to it before it will be lost. When information has entered STM and nothing is done to keep attention to the information it will decay fast. When strategies are applied to keep attention on the information however, for example by rehearsing the information, it can be kept online for as long as there is no interference to the rehearsal process by other information that attracts attention or needs attending to. The capacity of STM is limited and typically assessed by tasks that require subjects to recall increasingly longer sequences of items, e.g. digits or words. An individual's STM-span represents the longest list length that can be correctly recalled (Dehn, 2008). When individuals are allowed to use strategies to hold information in memory, such as rehearsal or chunking (e.g. organizing the number 3459682 into 345-96-82), they can keep up to seven items in memory (Miller, 1956). Without the use of strategies the capacity limit is about three to four items in adults (Cowan, 2001). During childhood there is an increase in the number of items children can keep in shortterm memory.

Working memory is another type of memory and refers to a system that is responsible for temporarily holding information in STM that at the same time is manipulated (Baddeley, 1992). The concept of WM became popular after the introduction of the WM model of Baddeley and Hitch (1974). This model states that STM can be divided in two modality specific subsystems termed the phonological loop and visuo-spatial sketchpad that contain two subcomponents for the temporary storage and rehearsal of verbal and visuo-spatial information respectively. A central executive mechanism, which is considered as the essence of WM, controls these two slave systems. The central executive is domain-free, and is, among oth- 
ers, responsible for linking the subsystems with each other, selecting strategies or allocating attention to the process of interest while irrelevant information is ignored. The latest component added to Baddeley's WM model is the episodic buffer, which is a temporary memory store controlled by the central executive. Its main function is to link together the several systems and most importantly, to link them with LTM. The episodic buffer presumably plays an important role in chunking information in STM by making use of LTM knowledge to group material into meaningful units (such as remembering the letters u-s-a-f-b-i as the words USA and FBI). Remembering information in such a deep way generally leads to higher recall scores compared to processing material in a shallow way during encoding (Craik \& Lockhart, 1972).

Just as STM, WM has limited capacity and information hold in WM is easily lost and should be rehearsed to prevent decay. The capacity of WM is typically assessed by using "complex" span tasks that require participants to store and process or manipulate increasingly large sequences of items until recall errors are made (Baddeley, Emslie, Kolodny, \& Duncan, 1998). STM tasks, in contrast, place no or minimal demands on processing and are often described as "storage-only" tasks. WM shows strong links with tasks that involve executive control operations, probably because they rely on highly similar brain networks, including the prefrontal cortex (e.g. (Bunge \& Wright, 2007; Klingberg, Forssberg, \& Westerberg, 2002). The capacity of working memory largely depends on the type of processing performed on the maintained material; when maintenance operations make great demands on executive control, capacity decreases. Because additional manipulation demands are often required in many higher-order cognitive skills, complex WM tasks also correlate stronger with higher-level cognitive skills, such as reasoning and intelligence, than simple storage tasks (e.g. (Engle, Tuholski, Laughlin, \& Conway, 1999; Kane, Hambrick, \& Conway, 2005). It is thus not surprising that especially WM is central to children's cognitive development because almost all of the learning activities that they encounter require both storage of information together with ongoing cognitive processing. WMC shows a lengthy developmental pattern and does usually not reach maturity until adolescence, i.e. by the age of 16 years (Siegel, 1994). This prolonged developmental course of WMC has been attributed to its dependency on the maturation of higher-order cortical brain areas necessary for implementing cognitive top-down control (e.g. a network of frontoparietal regions) that continue to develop into adolescence or even early adulthood (e.g. Crone, Wendelken, Donohue, van Leijenhorst, \& Bunge, 2006).

LTM refers to a memory system in which information can be stored permanently. All knowledge we have ever learned is stored in LTM, which consist of complex interconnected networks containing many links between concepts that are related to each other (Anderson, 1983). New information is attached and integrated into this existing network. When we need to use information stored in LTM, 
we should retrieve it again. The success of retrieval is largely dependent on how information was encoded and maintained in memory (Craik \& Lockhart, 1972). Strategies that increase the likelihood that information will be encoded in memory, for example when items are processed in terms of their meaning during encoding (e.g., deep, semantic encoding) are thus important for the learning process. When children are at an age that they can spontaneously use the semantic grouping strategy they indeed show improved memory performance (Bjorklund \& de Marchena, 1984). In contrast to both STM and WM, the capacity of LTM is nearly unlimited (Anderson, 2000). Most cognitive-psychological theories divide LTM into three categories, semantic memory, episodic memory, and procedural memory (Tulving, 1972). Semantic memory is especially important for academic learning because it contains all information we have ever learned, such as facts and concepts. Episodic memory refers to the memory of our own personal experiences, involves conscious thought and is declarative. Procedural memory refers to (implicit) knowledge about how to do something.

Children with learning disabilities frequently suffer from WM deficits and/or deficits in their semantic LTM. For example, children who perform poorly on WM tasks not only frequently experience impairments in reading and mathematics (Gathercole \& Alloway, 2008) they also often score below expected standards on curriculum assessment tests (Gathercole \& Pickering, 2000). Likewise, Geary (2004) proposed that deficits in semantic LTM, in specific in retrieving math information from it, are one of the three main causes of mathematical learning disabilities in children. Besides that WM and semantic LTM each seem to independently contribute to learning, there are several indications that WM is important for using or learning to use (semantic) LTM strategies during childhood. First, several studies demonstrated that, in adults, WM is important for successfully using the semantic grouping strategy (McNamara \& Scott, 2001; Turley-Ames \& Whitfield, 2003). Second, St Clair-Thompson, Stevens, Hunt and Bolder (2010) trained children in the use of memory strategies (among which semantic/categorical grouping) and found that this training significantly improved children's WMC. Finally, both WMC and more sophisticated memory strategies such as semantic grouping show a late development into adolescence (Bjorklund \& de Marchena, 1984; Bjorklund \& Jacobs, 1985; Siegel, 1994), possibly because they rely on a highly overlapping brain network (including fronto-parietal regions) that is not fully mature before early adulthood (Crone, Wendelken, Donohue, van Leijenhorst, \& Bunge, 2006; Miotto et al., 2006).

While the developmental pattern of strategy use is reasonably well established, the cognitive-(psychological) and biological mechanisms underlying different development phases of strategy development and how they relate to STM, WM or LTM are largely unknown. This is the main topic of this thesis. Before introduc- 
ing the topics of the four studies in this thesis, in the present chapter a background and review of the relevant literature will be provided.

\subsection{What is known about the typical development of short-term- and working memory-capacity?}

The development of STM-span has been studied by using simple span tasks that require subjects to store either verbal or visuo-spatial material in mind over short time periods. When children reach the age of 4 years they already show some rudimentary short-term memory functioning. One example of a frequently used verbal STM-span test is the digit span forward test in which subjects must recall increasingly larger sequences of digits (WISC-III; Wechsler, 1991). Throughout childhood there is an increase in the number of verbal information children can hold in their STM until adult levels are reached by the age of 12 years (Gathercole, 1999; Hulme, Thompson, Muir, \& Lawrence, 1984). The onset of rehearsal, which comes online at approximately 7 or 8 years of age, is thought to be one of the main causes for the developmental increases in verbal STM between age 4 and 12 (Gathercole \& Hitch, 1993). The increase in rehearsal ability around age 8 is in part caused by the enhanced verbal ability to articulate information at a faster rate so that material can be rehearsed more efficiently; this in turn further increases verbal STM-span (Roodenrys, Hulme, \& Brown, 1993). When children do not yet make use of verbal rehearsal, visual information enters the visuo-spatial sketchpad because the phonological loop cannot yet convert the visual information into phonological codes (Hitch, Halliday, Schaafstal, \& Schraagen, 1988). Studies investigating the development of visuo-spatial STM span often used visuo-spatial versions of verbal memory span tests, such as the corsi block test. In this test nine or ten blocks are randomly arranged on a wooden board; the subject's task is to touch the blocks in the sequence previously shown by the experimenter (Corsi, 1972). The length of the longest correctly reproduced sequence represents an individual's visuo-spatial memory span. Between the age of 5 to 11 , memory span for visuospatial material increases from approximately two to four items until adult levels are reached (Riggs, McTaggart, Simpson, \& Freeman, 2006). Thus both the capacity for temporarily maintaining verbal as well as visuo-spatial increases with age and is at mature levels by the end of childhood.

The development of WM is typically investigated by evaluating children's performance on "complex" WM tasks. As already mentioned, in contrast to STM, WMC shows a late development into adolescence (Dehn, 2008; Siegel, 1994). Several accounts have been proposed to explain the source of developmental improvements in WMC. Based on Daneman and Carpenter (1980) view that WM storage and processing requirements rely on the same pool of resources, Case, 
Kurland and Goldberg (1982) proposed that while the total amount of processing resources stays constant with age, there are developmental increases in the efficiency of processing activities (Gathercole, 1999). Thus as children get older they need less resources to support processing activities, which leads to increases in memory storage capacity. Another view suggests that improvements in selective attention with age are the major cause of WMC-increases (Engle, et al., 1999). Selective attention is important for proper WM functioning because it allows access and maintenance of only task relevant information in WM. In the context of Baddeley's central executive mechanism this raises the question to what extent executive control processes such as selective attention play a role in the late development of WM. Also executive functions such as selective attention, inhibition and response selection show a late development into adolescence (Bunge \& Wright, 2007; Casey et al., 1995; Diamond, 2002; Huizinga, Dolan, \& van der Molen, 2006; Luna \& Sweeney, 2004), which has been ascribed to the prolonged development of frontoparietal networks that are known to underlie various executive processes (Casey, Giedd, \& Thomas, 2000; Giedd et al., 1999; Gogtay et al., 2004; Luciana \& Nelson, 1998; Pandya, 1987). Interestingly, similar fronto-parietal networks are recruited during complex WM tasks (e.g. Crone, et al., 2006), suggesting that involved executive control operations may be an important factor in explaining the late development of WM.

In adults executive control functions have been divided in three distinct executive sub-processes: shifting, updating and inhibition (Miyake et al., 2000). St ClairThompson and Gathercole (2006) could only identify two separate sub-executive processes in 11- and 12- year-old children, namely updating and inhibition. Whereas updating refers to the continuous monitoring of incoming information and revising the items currently held in memory (Swanson, Howard, \& Saez, 2006), inhibition requires the ability to inhibit automatic or prepotent responses (e.g. Stroop, 1935). Until now little is known about how much the development of WMC is influenced by situations that do or do not require executive control processes like updating and interference control (which is a type of inhibition). This question was addressed in chapter 2 of this thesis.

Regarding LTM, in particular the development of semantic memory is important for the current thesis because this subsystem provides the basis for acquiring and retrieving all factual knowledge we have learned. Already in infancy developmental changes have been reported to take place in semantic memory, as shown by studies demonstrating that infants as young as 12-months can distinguish between certain different categories (Ross, 1980). Although infant's semantic memory is far from what it is in adulthood, these basic semantic memory abilities in early childhood are crucial for forming a semantic network. During the school years there is a substantial increase in semantic memory because children learn new facts and words on a daily basis. Developmental increases in semantic LTM 
also might provide a greater opportunity to apply memory strategies that make use of information stored in LTM, such as grouping items on the basis of category information stored in LTM or chunking information in more meaningful, familiar units.

\subsection{Most distinguished strategies in the developmental literature}

Two prominently used strategies are rehearsal and semantic grouping. Rehearsal refers to a process of repeating items over and over again (Gathercole, 1999). Rehearsal can either be subvocally or vocally. Semantic grouping is a memory strategy that refers to mentally grouping or organizing the to-be-learned information according to shared (semantic) features (Mandler, 1967).

For both the rehearsal and semantic grouping strategy, there are two main types that each show a different developmental pattern. Regarding rehearsal, children of 6 to 7 years of age spontaneously start to use the simplest form of it called single item rehearsal, meaning that only one item at a time is repeated (Kail, 1990). By the age of $9 / 10$ years, children are able to spontaneously rehearse together several items in groups (Guttentag, Ornstein, \& Siemens, 1987). This more advanced version is labelled cumulative rehearsal, and use of this strategy generally leads to higher improvements in memory performance than single item rehearsal (Schneider \& Pressley, 2013). The first use of the semantic grouping strategy is seen at the age of 9 years (Bjorklund \& de Marchena, 1984). At this stage children can only group information on semantic characteristics that are highly associated (e.g. are well-learned), such as the words dog and cat. Using this type of the grouping strategy is generally considered not to represent intentional strategy use since highly associated items are activated more or less automatically when they need to be remembered (Schneider \& Pressley, 1997). The grouping strategy for highly associated material is a precursor of the grouping strategy for material sharing low within-category associations with each other. According to the available literature, it is only by the age of 13 years that children begin to use this more intentional type of the semantic grouping strategy searching for semantic associations in the to-be-learned material themselves (e.g. when the items mouse and dolphin are grouped together in memory; (Bjorklund \& de Marchena, 1984; Bjorklund \& Jacobs, 1985). There are some suggestions from developmental studies that, before children show semantic grouping strategy use, they prefer to group to-be-learned material according to perceptual features such as color (Melkman, Tversky, \& Baratz, 1981).

From the above review it can be concluded that the acquisition of the rehearsal and semantic grouping memory strategy follows a prolonged time path that spans childhood and adolescence. During this developmental course, children do 
not suddenly start to use the above (as well as other) strategies, but typically pass through several phases on their way to successful strategy use (Miller \& Seier, 1994). Initially, children are said to be mediation deficient, meaning that they cannot spontaneously use strategies and can also not be instructed or trained to do so. The second phase is named the production deficiency phase in which children do not spontaneously use the strategy, but are only able to successfully use strategies when instructed in their use. Then, in the third phase, children have a utilization deficiency indicating that they show spontaneous use of memory strategies but do not yet benefit from this in terms of better memory (higher recall). In the last phase, children can spontaneously produce strategies and profit from its use (i.e. show improved memory performance compared to when the strategy is not used). Whereas these developmental phases have been elaborately described in the developmental literature, the cognitive mechanisms that underlie developmental improvements in memory strategy use are largely unknown. As will become clear in the next paragraphs, several studies indicate that working memory capacity might be one important mechanism involved. Before discussing these studies, first a short overview will be given about which memory systems are involved in rehearsal and semantic grouping.

\subsection{How are the different memory systems involved in the rehearsal and semantic grouping strategy?}

Before information can be maintained and rehearsed in memory, first attention has to be paid to it so that it can be selected to enter STM. After the information of interest has been converted into a memory trace, the rehearsal process can be initiated. The part of the memory system that rehearses verbal information is the phonological loop (Baddeley \& Hitch, 1974), which consists of two parts: the phonological store and the articulatory rehearsal component. In the former the memory traces are stored for up to several seconds before they will decay unless the information is rehearsed by the articulatory rehearsal component. In some situations, for example when children listen to the teacher explaining the lesson, new input has to be incorporated into the existing memory trace. This is accomplished by the process of updating. With rehearsal, information can be maintained as long as possible when nothing happens that interrupts the rehearsal process. In cases where distracting information has to be ignored or filtered out, interference control is required to keep attention on the material in memory while at the same time the irrelevant information is suppressed so that it cannot enter the memory trace or disrupt the rehearsal process. All these additional cognitive activities (i.e. updating and interference control), besides rehearsal, require central executive resources and constitute WM. Also when load approaches or exceeds the capacity 
of the short-term stores, executive control processes are needed next to those of the domain-specific buffers to keep the information in memory (Cowan, 2001). Neurobiological studies have demonstrated that the prefrontal cortex not only shows increased activation in tasks that require concurrent storage and manipulation (i.e. executive control operations), but is also involved in memory tasks in which the to-be-stored material exceeds the capacity of STM (Rypma, Prabhakaran, Desmond, Glover, \& Gabrieli, 1999). Although rehearsal thus consumes some WMC and requires PFC activation, studies have shown that with practice, at least in adults, the rehearsal process can be carried out relatively effortless (Cowan, 2008). The above described more simple type of rehearsal that does not depend on LTM, is called maintenance rehearsal. Since maintenance rehearsal involves short-term storage only, this is not an effective strategy to transfer information to LTM. If we want to store information more permanently in memory, we must perform more elaborative cognitive operations on the incoming information, which involves making information more meaningful (Craik \& Lockhart, 1972). A piece of information is more meaningful than another if a larger number of connections can be made between it and other information already in long-term memory. Examples of elaborative encoding strategies are creating interactive images of the information, chunking information in more meaningful units or grouping material on similar semantic categories. Many behavioral studies have supported the hypothesis that memory performance benefits from elaborative processing (deep processing) during encoding, compared to when information is analyzed in terms of perceptual features (shallow encoding) (Craik, 2002). This phenomenon has also been termed the levels-of-processing effect, and refers to the notion that deep encoding enriches the memory representation of an item by activating many aspects of its meaning and linking it to an already existing network of semantic relations. The deeper information is processed during encoding, the stronger and longer lasting the resulting memory traces and the greater the likelihood that information will be correctly remembered. Elaborative encoding strategies might be especially helpful to store larger amounts of information because in this way information is stored in a more structured, organized way so that WM-load is decreased (Cowan, 1997). Rehearsal of deeply encoded information is also referred to as elaborative rehearsal (Craik \& Lockhart, 1972). Because WM is the mental workspace (i.e. in specific the episodic buffer; Baddeley, 2000) in which information from different memory systems is combined, it seems likely that WM plays a key role in elaborative encoding because this entails connecting new information to information already stored in LTM. The next paragraph will review available evidence for links between WM and one type of elaborative encoding, namely semantic grouping, which is the focus of the current thesis. 


\subsection{Evidence for a possible role of WM in the semantic grouping strategy}

\subsubsection{Evidence for a mediating role of working memory capacity in the effective application of semantic grouping strategies in adults}

There are several experimental studies in adults that have suggested that WMC plays an important role in the application of semantic grouping strategies. These studies will be shortly reviewed here. In a study by Kaakinen \& Hyona (2007) it was investigated in two experiments if individuals with a low, medium and high memory WM-span differed in the strategies they used in the reading span test. In this test subjects are required to read series of sentences out loud and to recall the last word of each sentence in serial order (Daneman \& Carpenter, 1980). In particular the results of experiment 2 are relevant for the present thesis, showing that individuals with a high WM span reported to use more effective memory encoding strategies (semantic elaboration) than individuals with a low span (using rehearsal). In this study participants were divided in different memory span groups on the basis of the total number of words recalled in the reading span test. Relations between WMC and semantic strategy use have also been reported during memory retrieval (Rosen \& Engle, 1997; Schelbe, Therriault, \& Miller, 2012; Unsworth, Brewer, \& Spillers, 2013). All these studies investigated whether individuals with high or low WM capacity used different retrieval strategies. A category fluency test was used in which subjects had to generate as many words as possible from a given category (e.g. animals) within a certain time limit. One of the outcome measures in this test is the number and size of clusters of semantic subcategories that participants produce (e.g. all mammals). Collectively, the studies of Rosen and Engle (1997), Schelbe et al. (2012) and Unsworth et al. (2013) demonstrate that, compared to individuals with low WMC, high WMC individuals had higher recall due to the generation of more and larger semantic clusters and the use of different retrieval strategies.

From the above it can be concluded that there is empirical evidence for relations between WMC and the skill to successfully apply semantic grouping strategies in adults, both during encoding and retrieval. Until now not much is known about whether similar WMC-semantic strategy use connections exist in childhood.

\subsubsection{Support from developmental research for a link between WMC and semantic grouping strategy use in childhood}

Only few studies examined developmental relations between WMC and semantic grouping strategy use. The first indications for this link were provided by developmental research using a so called dual-task methodology in which performance on a secondary task (e.g. finger-tapping test) is evaluated as a function of perfor- 
mance on a primary task that requires mental resources (e.g. free-recall task) (Bjorklund \& Harnishfeger, 1987). The finding that older children experienced less interference from the secondary task than younger children led Bjorklund and Harnisfeger (1987) to conclude that younger children fail to use memory strategies because their use would consume too much of their yet limited cognitive resources. One possible problem with interpreting the results of dual-task studies is that the developmental decrease in secondary task disruption might be (partly) attributed to development improvements in resisting and inhibiting interference due to performing two tasks at the same time (Brainerd \& Reyna, 1989). Further, performance on a finger tapping test might not be a pure measure of an individual's memory capacity since several other (cognitive) processes have been suggested to be involved in the execution of such tasks (e.g. motor speed and motor control; Christianson \& Leathem, 2004).

In a series of longitudinal studies Schneider and colleagues more systematically studied the WMC-strategy use relation in children. In their first study, it was examined if WMC differed between consistently strategic children and utilization deficient children (Schneider, Kron, Hunnerkopf, \& Krajewski, 2004). Consistently strategic children included children who applied the semantic grouping strategy at encoding (i.e. the sorting strategy) at two consecutive time points and had high recall scores. Utilization deficient children, in contrast, referred to children who showed increased use of the sorting strategy but who had no corresponding improvements in recall scores. WMC was reported to be higher in consistently strategic compared to utilization deficient children. In a subsequent study, Kron-Sperl, Schneider and Hasselhorn (2008) found that individual differences in STM, but not WM, were significant predictors of recall scores in children who showed spontaneous use of the semantic grouping strategy. Absence of a WMC-semantic grouping strategy use relation in this study may be because children had to study highly associated items, which might possibly require less working memory capacity as they are automatically activated from memory.

This short review illustrates that only little research has been done into the role of WMC in the use of semantic grouping strategies in children and clearly more research is needed to investigate this. Chapters 3 and 4 of this thesis focus on this topic.

\subsection{Why use ERPs to study the neural basis of elaborative memory encoding?}

From the previous paragraphs it becomes clear that the strategies used during the encoding phase of memory are an important determinant of how well material can be learned or remembered. The previous sections also show that the developmen- 
tal stages through which children pass before they become strategic are rather well established (although primarily using cross-sectional designs), but relatively little is known about the mechanisms underlying progression to a next developmental stage. Brain research, and in particular ERPs with its precise temporal resolution, are suitable to disentangle the cognitive sub-processes involved in memory strategy use, and could help to eventually answer which specific processes contribute to the late development of certain memory strategies. ERPs are a noninvasive method for measuring brain activity during performance of cognitive tasks. The ERP is the portion of the electroencephalogram (EEG) that is timelocked to a particular event (e.g. a stimulus) (Handy, 2005). Because ERPs have a temporal resolution in the millisecond range, as opposed to imaging methods as fMRI or PET, they provide a precise measure of online cognitive processing. ERPs might be especially helpful in the area of memory research because (1) cognitive processing in memory tasks usually proceeds through several different stages (encoding, maintenance, retrieval) that are sometimes difficult to separate if only behavioural tasks are used and (2) in particular during memory encoding cognitive processes are of relatively short duration and follow each other rapidly in time. Further, ERPs can be used in young children in future studies. Because ERP research into the neural basis of elaborative encoding is relatively scarce, in this thesis we first examined the ERP correlates underlying semantic grouping in adults.

\subsection{The neural basis of elaborative encoding, in specific of semantic grouping}

As already mentioned, when elaborative strategies are implemented during the encoding phase this means that the to-be-learned information is processed in a more meaningful way which typically results in better memory performance compared to when no or shallow strategies such as rehearsal are applied to incoming information. In the current thesis the neural basis of one type of elaborative encoding (i.e. semantic grouping) was examined by using ERPs. Before reviewing the relevant ERP literature, first a short overview will be given about the brain regions supporting elaborative encoding. Several recent studies have shown that when individuals learn to use elaborative encoding strategies, this requires capacity and prefrontal cortex (PFC) activation (Kirchhoff, Anderson, Barch, \& Jacoby, 2012; Miotto, et al., 2006; Nyberg et al., 2003). Nyberg and colleagues (2003) and Miotto et al. (2003) investigated for the first time which brain regions underlie the acquirement of elaborative encoding strategies. In the study by Nyberg et al. (2003) younger and older adults were trained in using the method of loci strategy which involves attaching the to-be-learned information to different locations of a familiar 
route (e.g. along one route to work). It was examined which brain regions accompany possible training-related improvements, that are often seen when this strategy is used. Younger adults as well as those older adults who benefitted from the training showed increased activity during memory encoding in occipito-parietal brain regions, and the former also in frontal brain areas. Miotto et al. (2003) trained young adults in the use of a semantic grouping strategy and changes in brain activation patterns before and after training were compared. The semantic grouping strategy training led to increased recall and semantic clustering scores, the latter reflecting to what extent the items had been grouped on semantic category during recall. At the brain level there was increased activation in bilateral dorsolateral PFC and orbitofrontal cortex after vs. before training. Finally, Kirchhoff and co-workers (2012) examined if learning to use three semantic strategies (sentence generation, pleasantness and personal relevance) improved memory performance and how this affected older adult's brain activation patterns. These authors found that only after training older adults spontaneously used semantic encoding strategies, which led to increased memory recognition performance so that it no longer differed from that of younger adult's recognition performance. After training there also was increased activity in prefrontal and left lateral temporal regions during encoding.

Other studies report that also when elaborative encoding strategies are applied to incoming information, activity in (among others) the prefrontal cortex increases (Bor, Cumming, Scott, \& Owen, 2004; Bor, Duncan, Wiseman, \& Owen, 2003; Bor \& Owen, 2007; Kirchhoff \& Buckner, 2006). Bor and colleagues (2003, 2004,2007 ) conducted several studies to investigate by means of fMRI which brain regions support the use of chunking, which is a strategy whereby material is grouped in more compact units. These authors reported that memory performance only improved on trials that allowed chunking vs. trials that did not allow chunking. This was found irrespective to which type of material the chunking strategy was applied (verbal or nonverbal) or the specific recoding process required (mathematical or mnemonic strategies). In an fMRI study by Kirchhoff and Buckner (2006), subjects were presented with unrelated, interacting object pairs (e.g. a big standing on a key) which they had to study for a subsequent memory test of which they received no detailed information. Following a recognition test subjects had to indicate by means of a self-report questionnaire which strategies they had used during learning. This revealed that verbal elaboration and visual imagery were two main strategies used during encoding, which was related to subject's performance on the memory test. The fMRI data showed that while using verbal elaboration activated left inferior frontal regions, left occipito-temporal brain regions were active during visual imagery. Finally, an interesting study by Innocenti et al. (2010) used rTMS, which allows to draws causal relations between the level of activation of a specific brain region and the cognitive process of interest, to ex- 
amine if the left DLPFC is functionally needed to successfully remember deeply encoded material. In this study online rTMS was applied when subjects encoded words in a deep (discriminating between living and nonliving things) and shallow way (deciding whether the word contained the letter "e"). Retrieval (memory recognition) was subsequently tested without rTMS interference. This study showed that stimulation of the left DLPFC eliminated the beneficial effect of deep semantic analyses during encoding on recognition accuracy rates. This finding indicates that the left DLPFC is specifically involved in encoding material in a semantic way during encoding.

Thus, the above review shows that a network including prefrontal and posterior brain regions supports the use of various elaborative encoding strategies. Two studies using PET specifically examined which brain regions are involved in semantic grouping (Fletcher, Shallice, \& Dolan, 1998; Savage et al., 2001). In the study by Savage et al. (2001) participants heard 24 sequentially presented words while they underwent PET, which they had to recall later in three different conditions. In the spontaneous condition, the words belonged to four different semantic categories, but were presented to participants in a mixed order. Participants were not informed about the semantic relationships between items. Subjects received a similar word list (including different pictures) in the directed condition. In this condition participants were explicitly instructed to search for the four categories and to mentally organize the words into these categories to enhance their memory performance. In the unrelated condition words were not semantically related to each other. These authors found that the left inferior prefrontal cortex (LIPC) was especially active in the directed condition when compared to the spontaneous and unrelated condition. Further, also greater activation in the left dorsolateral PFC (DLPFC) for directed compared to both spontaneous and unrelated conditions was found. Fletcher et al. (1998) used a comparable design and reported similar relations between DLPFC activation and semantic grouping.

So far only a limited number of studies used ERPs to investigate the temporal course of cognitive processes involved in semantic grouping. One study that provides important information about this is a study performed by Blanchet, Gagnon and Bastien (2007). Here, participants had to learn sequentially presented words that differed in the degree of semantic organization required. In unrelated conditions, the words did not share any semantic relationship with each other. Both in spontaneous and guided conditions, the to-be-learned words belonged to four different categories, but only in the latter condition subjects were explicitly required to group the words on their respective semantic category (in this condition also the names of the corresponding semantic categories were provided). The earliest component that was found to be modulated was the P200, which was increased over fronto-central regions in guided vs. spontaneous vs. unrelated conditions. Following research implicating the P200 in attentional processing during 
deep encoding, the authors suggested that the P200 was largest in the guided condition because in this condition the semantic categories were known in advance so that subjects could quickly allocate their attention to the semantic features of the words. Then, a late positive component (LPC) over centro-parietal regions was elicited between 400-800 ms in both the guided and spontaneous conditions compared to the unrelated condition. On the basis of others and their own findings the authors suggested that the LPC indexes voluntary associative processes involved in attempting to link words together that belong to similar semantic categories. Finally, the spontaneous condition (vs. the unrelated and guided condition) elicited a right-frontal sustained slow wave activity between 600-1200 ms; a similar frontal slow wave was also found between 1200-1800 ms for the spontaneous condition vs. unrelated contrast. Blanchet et al. concluded that the right frontal slow wave indexed the degree of self-initiation involved in application of the semantic organizational strategy, which was based on their finding that the sustained right frontal slow wave was only increased in the spontaneous condition. Another line of research that is relevant for the current thesis, although not directly manipulating semantic grouping strategy use, are studies that examined which ERP components are modulated when information about specific semantic categories needs to be retrieved from LTM (Kiefer, 2001, 2005).These studies reported modulations of a negative component around $400 \mathrm{~ms}$ at fronto-central, centro-parietal and occipitotemporal electrodes when specific information about object categories had to be retrieved from semantic LTM (Kiefer, 2001, 2005).

An important notion about the above studies is that subjects did not receive any instructions on how to memorize the information in unrelated/unstructured trials, which might have encouraged them to use rehearsal to remember the items in these trials. Also encoding activity might have been mixed with rehearsal activity in structured trials in fMRI/PET studies because of lower temporal resolution. This means that the reported effects of these studies might (partly) reflect the involvement of more simple strategies such as rehearsal. Indeed, there is great overlap in the brain regions involved in semantic grouping and rehearsal, including left prefrontal areas (e.g. Smith \& Jonides, 1998). In chapter 5 of this thesis a study is reported that examined if the ERP components earlier related to semantic grouping are indeed specifically involved in semantic grouping, or are also related to rehearsal.

After information has been encoded in a deep (semantic) way during encoding, this material should be maintained in memory during a delay period in most memory tasks which will probably require different types of rehearsal. Maintaining in memory semantically grouped pictures most likely requires elaborative rehearsal operations, while maintenance of pictures that are only analysed in a shallow way during encoding (rehearsal) will involve simple rote rehearsal processes. Several studies using fMRI examined which brain regions support mainte- 
nance of material in memory in an organized way (Prabhakaran, Narayanan, Zhao, \& Gabrieli, 2000; Wendelken, Bunge, \& Carter, 2008). Prabhakaran and coworkers (2000) asked subjects to remember letters or spatial locations, which were presented together (as belonging to the same object) or separately. Activity in prefrontal brain regions only increased in conditions in which the letters and locations were integrated and memorized together. In a study by Wendelken et al. (2008), two experiments were conducted that included WM-delay tasks involving maintenance of organized or unorganized material. The organized condition was of a different nature in the two experiments; while in experiment 1 a set of items had to be divided into groups, in experiment 2 items had to be organized on the basis of certain relationships. In both experiments activity in the dorsolateral cortex increased particularly in organized vs. unorganized conditions. Finally, ERP research showed that memory maintenance operations elicit so-called slow wave components that become more negative with increasing task difficulty or mental load (Kiss, Watter, Heisz, \& Shedden, 2007; Rosler, Heil, \& Roder, 1997). For instance, Kiss et al. (2007) found that negative slow waves above left frontal regions increased in amplitude during WM-updating vs. both WM-maintenance and control tasks. If elaborative vs. rote rehearsal processes give rise to distinct slow wave components is a relatively unexplored issue so far. This was also addressed in the study presented in chapter 5 .

\subsection{Aims and outline of the present thesis}

The main aim of the current thesis is to enhance our understanding of how working memory capacity and semantic grouping strategy use develops during childhood and to get more insight into the role that WMC plays in the development of strategy use (especially semantic grouping). The specific research aims in the different chapters are the following:

- To investigate how the development of WMC from age 6 to 12 years (divided in three age groups, i.e. 6-7, 8-9 and 10-12 year-old-children) is influenced by varying executive control demands of the task.

- To investigate (a) if WMC is important for the development of successful semantic grouping strategy use in 6-12 year-old children and (b) and if a single nondirective prompt towards strategy use can lead to successful strategy use in children that do not yet spontaneously use the strategy.

- To examine the longitudinal development of semantic grouping strategy use. More specifically, it was investigated (a) through which phases children pass before they are able to successfully use the semantic grouping strategy, (b) and if children who only recently acquired the semantic grouping strategy after prompting show strategy transfer to a new task when not prompted. 
- To explore, by means of the ERP technique, which neural correlates support the use of semantic grouping in adults.

Chapter 2 reports on an experiment that examined how much the development of WMC is dependent on executive control functions (i.e. interference control), that show a late development into adolescence (Bunge \& Wright, 2007). To investigate this, 6-12 year-old children and one group of adults performed an N-Back task including three load conditions (0-, 1-, 2-back). While the 1-back task required storage of one letter only, in the 2-back task also memory updating of several letters was required as well as controlling interference of the intervening letter. To establish the involvement of more executive control processes in the 2-back task, all subjects also performed a flanker task that measured the children's interference control ability. Based on previous findings that both WMC and the ability to resolve interference are highly dependent on fronto-parietal regions that follow a prolonged developmental pattern into adolescence (Bunge et al., 2001), it was expected that only performance in the 2-back task would show delayed development into adolescence. In Chapter 3 the developmental pattern of semantic memory grouping strategy use was examined. This study had two main aims: 1) to examine the role of WMC in the development of semantic grouping strategies and 2) to investigate the age at which children are able to spontaneously use this strategy, and if a single nondirective prompt can lead to successful use of it in nonstrategic children. In this study 6-12 year-old children performed two sort-recall tasks (one without and one with a grouping instruction) that measure the extent to which pictures are grouped on semantic category during the study as well as recall phase. Digit span tests (forward and backward) were also administered to obtain a measure of a child's STM and WMC, respectively. The role of STM/WMC in semantic strategy use development was tested in two ways, (1) by performing a mediation analysis that examined if STM and/or WMC significantly mediated the relation between semantic strategy use and recall and (2) by performing a between subject analysis that tested if STM/WMC was higher in strategic children compared to nonstrategic children. Based on several indications mentioned earlier in this chapter, it was expected that also in children, WMC plays an important role in successful application of the semantic grouping strategy. Chapter 4 was a longitudinal follow-up study of the study described in chapter 3. A subset of children included in the cross-sectional study described in chapter 3 participated in this longitudinal study and were tested again 1,5 years later. As described earlier in this introduction, children have been shown to pass through several phases before they show spontaneous and consistent strategy use. However, this has mainly been inferred from cross-sectional data and longitudinal studies are lacking so far, which is however important in order to establish if the developmental progression from one phase to another is also observed within the same subject groups. The first aim of 
this longitudinal study was to investigate whether 6-7-year-olds first pass through the mediation deficiency (MD) phase before they enter the production deficiency (PD) phase at the age of 8-9, and if 8-9-year-olds first pass through the PD phase before they are able to consistently use the semantic grouping strategy at the age of 10-12. To this end, children aged 6-7 and 8-9-years performed two sort-recall tasks (one without and one with a grouping instruction) at two time points separated 1,5 years from each other. Another gap in the developmental literature is that relatively little is known about if children who recently acquired the semantic grouping strategy are able to transfer this strategy to a new task. This was another aim of this study, in specific it was investigated whether PD children that only showed successful strategy use after prompting and children who used the strategy spontaneously, differed in strategy transfer to a sort-recall task including new pictures and new semantic categories at time point 2. In Chapter $\mathbf{5}$ a study is described that investigated the neural correlates of semantic grouping strategy use and maintenance in adults using the ERP technique and using tasks most similar to those used in chapters 3 and 4 in the developmental studies. Only very few ERP studies investigated the temporal brain correlates underlying strategy use and these studies did not show whether ERP activity was specifically linked to semantic grouping or rehearsal processes, the latter being used in almost all memory tasks and thus likely overlapping in time with strategy use. In this study participants encoded four simultaneously presented pictures of objects (S1) in two different conditions while EEG was recorded. In related trials these four pictures were from two different semantic categories, and subjects were explicitly instructed to group these pictures on their corresponding semantic categories during encoding. In unrelated trials, the four pictures in S1 belonged to four different semantic categories, and subjects were explicitly instructed to rehearse these pictures. In contrast to previous studies, this latter explicit rehearse instruction was given to experimentally control the strategies used by subjects in the unrelated condition. This allowed us to investigate if the ERP-components previously associated with semantic grouping (i.e. P200, N400, LPC and late sustained positive wave) are indeed associated specifically with semantic grouping (deep encoding) or might also reflect involved rehearsal processes (shallow encoding). In this study it was also examined if different ERP slow waves are elicited during maintenance (rehearsal) of semantically grouped vs. ungrouped material. To this end, following encoding, subjects were also asked to maintain the four pictures in memory during a delay period until a probe occurred (S2). 


\section{References}

Anderson, J. R. (1983). Retrieval of information from long-term memory. Science, 220, 25-30.

Anderson, J. R. (2000). Learning and memory: An integrated approach. New York: John Wiley \& Sons.

Baddeley. (2000). The episodic buffer: a new component of working memory? Trends in Cognitive Sciences, 4, 417-423.

Baddeley, \& Hitch, G. J. (1974). Working memory. In G. A. Bower (Ed.), Recent advances in learning and motivation (Vol. 8, pp. 47-89). New York: Academic Press.

Baddeley, A. (1992). Working memory. Science, 255, 556-559.

Baddeley, A., Emslie, H., Kolodny, J., \& Duncan, J. (1998). Random generation and the executive control of working memory. Quarterly Journal of Experimental Psychology Section a-Human Experimental Psychology, 51, 819-852.

Bjorklund, D. F., \& de Marchena, M. R. (1984). Developmental shifts in the basis of organization in memory: The role of associative versus categorical relatedness in children's free recall. Child Development, 55, 952-962.

Bjorklund, D. F., \& Harnishfeger, K. K. (1987). Developmental differences in the mental effort requirements for the use of an organizational strategy in free recall. Journal of Experimental Child Psychology, 44, 109-125.

Bjorklund, D. F., \& Jacobs, J. W. (1985). Associative and categorical processes in children's memory: The role of automaticity in the development of organization in free recall. Journal of Experimental Child Psychology, 39, 599-617.

Blanchet, S., Gagnon, G., \& Bastien, C. (2007). Event-related potential study of dynamic neural mechanisms of semantic organizational strategies in verbal learning. Brain Research, 1170, 59-70.

Bor, D., Cumming, N., Scott, C. E., \& Owen, A. M. (2004). Prefrontal cortical involvement in verbal encoding strategies. The European journal of neuroscience, 19, 3365-3370.

Bor, D., Duncan, J., Wiseman, R. J., \& Owen, A. M. (2003). Encoding strategies dissociate prefrontal activity from working memory demand. Neuron, 37, 361-367.

Bor, D., \& Owen, A. M. (2007). A common prefrontal-parietal network for mnemonic and mathematical recoding strategies within working memory. Cerebral Cortex, 17, 778-786.

Brainerd, C. J., \& Reyna, V. F. (1989). Output-interference theory of dual task deficits in memory development. Journal of Experimental Child Psychology, 47, 1-18.

Bunge, S. A., Ochsner, K. N., Desmond, J. E., Glover, G. H., \& Gabrieli, J. D. (2001). Prefrontal regions involved in keeping information in and out of mind. Brain : a journal of neurology, 124, 20742086.

Bunge, S. A., \& Wright, S. B. (2007). Neurodevelopmental changes in working memory and cognitive control. Current Opinion in Neurobiology, 17, 243-250.

Case, R., Kurland, D. M., \& Goldberg, J. (1982). Operational efficiency of short-term memory span. Journal of Experimental Psychology, 33, 386-404.

Casey, B. J., Cohen, J. D., Jezzard, P., Turner, R., Noll, D. C., Trainor, R. J., et al. (1995). Activation of prefrontal cortex in children during a nonspatial working memory task with functional MRI. Neuroimage, 2, 221-229.

Casey, B. J., Giedd, J. N., \& Thomas, K. M. (2000). Structural and functional brain development and its relation to cognitive development. Biological psychology, 54, 241-257.

Christianson, M. K., \& Leathem, J. M. (2004). Development and standardisation of the computerised finger tapping test: Comparison with other finger tapping instruments. New Zealand Journal of Psychology, 33, 44-49.

Corsi, P. M. (1972). Human memory and the medial temporal region of the brain.

Cowan, N. (1997). The development of memory in childhood. East Sussex, UK: Psychology Press.

Cowan, N. (2001). The magical number 4 in short-term memory: a reconsideration of mental storage capacity. Behavioral and brain sciences, 24, 87-114; discussion 114-185. 
Cowan, N. (2008). What are the differences between long-term, short-term, and working memory? Progress in brain research, 169, 323-338.

Craik, F. I. M. (2002). Levels of processing: Past, present ... and future? Memory, 10, 305-318.

Craik, F. I. M., \& Lockhart, R. S. (1972). Levels of processing: A framework for memory research. Journal of verbal learning and verbal behavior, 11, 671-684.

Crone, E. A., Wendelken, C., Donohue, S., van Leijenhorst, L., \& Bunge, S. A. (2006b). Neurocognitive development of the ability to manipulate information in working memory. Proceedings of the National Academy of Sciences of the United States of America, 103, 9315-9320.

Daneman, M., \& Carpenter, P. A. (1980). Individual differences in working memory and reading. Journal of verbal learning and verbal behavior, 19, 450-466.

Dehn, M. J. (2008). Working memory and academic learning: Assesment and intervention. Hoboken, New Jersey: Wiley \& Sons, Inc.

Diamond, A. (2002). Normal development of prefrontal cortex from birth to young adulthood: Cognitive functions, anatomy, and biochemistry. In D. T. Stuss, Knight, R.T. (Ed.), Principles of frontal lobe function. New York: Oxford University Press.

Engle, R. W., Tuholski, S. W., Laughlin, J. E., \& Conway, A. R. (1999). Working memory, short-term memory, and general fluid intelligence: a latent-variable approach. Journal of experimental psychology. General, 128, 309-331.

Fletcher, P. C., Shallice, T., \& Dolan, R. J. (1998). The functional roles of prefrontal cortex in episodic memory. I. Encoding. Brain : a journal of neurology, 121, 1239-1248.

Gathercole, S. E. (1999). Cognitive approaches to the development of short-term memory. Trends in Cognitive Sciences, 3, 410-419.

Gathercole, S. E., \& Alloway, T. P. (2008). Working memory and classroom learning. In S. K. Thurman \& C. A. Fiorello (Eds.), Applied cognitive research in $K-3$ classrooms (pp. 17-40). New York: Routledge/Taylor \& Francis.

Gathercole, S. E., \& Hitch, G. J. (1993). Developmental changes in short-term memory: A revised working memory perspective. In A. Collins, S. E. Gathercole, M. A. Conway \& P. E. Morris (Eds.), Theories of memory (pp. 189-210). Hove, UL: Erlbaum.

Gathercole, S. E., \& Pickering, S. J. (2000). Working memory deficits in children with low achievements in the national curriculum at 7 years of age. The British journal of educational psychology, 70, 177194.

Geary, D. C. (2004). Mathematics and learning disabilities. Journal of learning disabilities, 37, 4-15.

Giedd, J. N., Blumenthal, J., Jeffries, N. O., Castellanos, F. X., Liu, H., Zijdenbos, A., et al. (1999). Brain development during childhood and adolescence: a longitudinal MRI study. Nature Neuroscience, 2, 861-863.

Gogtay, N., Herman, D., Ordonez, A., Giedd, J., Hayashi, K., Greenstein, D., et al. (2004). Dynamic mapping of cortical brain development in pediatric bipolar illness. Neuropsychopharmacology : official publication of the American College of Neuropsychopharmacology, 29, S82-S82.

Guttentag, R. E., Ornstein, P. A., \& Siemens, L. (1987). Children's spontaneous rehearsal: Transition's in strategy acquistion. Cognitive Development, 2, 307-326.

Handy, T. C. (2005). Event-related potentials: A methods handbook. Cambridge, Massachusetts: MIT press.

Hitch, G. J., Halliday, S., Schaafstal, A. M., \& Schraagen, J. M. (1988). Visual working memory in young children. Memory \& Cognition, 16, 120-132.

Huizinga, M., Dolan, C. V., \& van der Molen, M. W. (2006). Age-related change in executive function: developmental trends and a latent variable analysis. Neuropsychologia, 44, 2017-2036.

Hulme, C., Thompson, N., Muir, C., \& Lawrence, A. (1984). Speech rate and the development of shortterm memory span. Journal of Experimental Child Psychology, 38, 241-253.

Innocenti, I., Giovannelli, F., Cincotta, M., Feurra, M., Polizzotto, N. R., Bianco, G., et al. (2010). Eventrelated rTMS at encoding affects differently deep and shallow memory traces. Neuroimage, 53, 325-330. 
Kaakinen, J. K., \& Hyona, J. (2007). Strategy use in the reading span test: an analysis of eye movements and reported encoding strategies. Memory, 15, 634-646.

Kail, R. (1990). The development of memory in children (3rd ed.). New York: Freeman.

Kane, M. J., Hambrick, D. Z., \& Conway, A. R. (2005). Working memory capacity and fluid intelligence are strongly related constructs: comment on Ackerman, Beier, and Boyle (2005). Psychological Bulletin, 131, 66-71; author reply 72-65.

Kiefer, M. (2001). Perceptual and semantic sources of category-specific effects: event-related potentials during picture and word categorization. Memory \& Cognition, 29, 100-116.

Kiefer, M. (2005). Repetition-priming modulates category-related effects on event-related potentials: further evidence for multiple cortical semantic systems. Journal of Cognitive Neuroscience, 17, 199-211.

Kirchhoff, B. A., Anderson, B. A., Barch, D. M., \& Jacoby, L. L. (2012). Cognitive and neural effects of semantic encoding strategy training in older adults. Cerebral Cortex, 22, 788-799.

Kirchhoff, B. A., \& Buckner, R. L. (2006). Functional-anatomic correlates of individual differences in memory. Neuron, 51, 263-274.

Kiss, I., Watter, S., Heisz, J. J., \& Shedden, J. M. (2007). Control processes in verbal working memory: an event-related potential study. Brain Research, 1172, 67-81.

Klingberg, T., Forssberg, H., \& Westerberg, H. (2002). Training of working memory in children with ADHD. Journal of Clinical and Experimental Neuropsychology, 24, 781-791.

Kron-Sperl, V., Schneider, W., \& Hasselhorn, M. (2008). The development and effectiveness of memory strategies in kindergarten and elementary school: Findings from the Wurzburg and Gottingen longitudinal memory studies. Cognitive Development, 23, 79-104.

Luciana, M., \& Nelson, C. A. (1998). The functional emergence of prefrontally-guided working memory systems in four- to eight-year-old children. Neuropsychologia, 36, 273-293.

Luna, B., \& Sweeney, J. A. (2004). The emergence of collaborative brain function: FMRI studies of the development of response inhibition. Annals of the New York Academy of Sciences, 1021, 296-309.

Mandler, G. (1967). Organization and memory In K. W. Spence, Spence, J.T. (Ed.), The psychology of learning and motivation (Vol. 1, pp. 327-372). New York: Academic Press.

McNamara, D. S., \& Scott, J. L. (2001). Working memory capacity and strategy use. Memory \& Cognition, $29,10-17$.

Melkman, R., Tversky, B., \& Baratz, D. (1981). Developmental trends in the use of perceptual and conceptual attributes in grouping, clustering, and retrieval. Journal of Experimental Child Psychology, 31, 470-486.

Miller, G. A. (1956). The magical number seven plus or minus two: some limits on our capacity for processing information. Psychological Review, 63, 81-97.

Miller, P. H., \& Seier, W. L. (1994). Strategy utilization deficiencies in children:When, Where and Why. In H. W. Reese (Ed.), Advances in child development and behavior (Vol. 25, pp. 105-156). San Diego: Academic Press.

Miotto, E. C., Savage, C. R., Evans, J. J., Wilson, B. A., Martins, M. G., Iaki, S., et al. (2006). Bilateral activation of the prefrontal cortex after strategic semantic cognitive training. [Research Support, Non-U.S. Gov't]. Human Brain Mapping, 27, 288-295.

Miyake, A., Friedman, N. P., Emerson, M. J., Witzki, A. H., Howerter, A., \& Wager, T. D. (2000). The unity and diversity of executive functions and their contributions to complex "frontal lobe" tasks: A latent variable analysis. Cognitive Psychology, 41, 49-100.

Nyberg, L., Sandblom, J., Jones, S., Neely, A. S., Petersson, K. M., Ingvar, M., et al. (2003). Neural correlates of training-related memory improvement in adulthood and aging. Proceedings of the National Academy of Sciences of the United States of America, 100, 13728-13733.

Pandya, D. N., Barnes, C.L. (1987). The frontal lobes revisited. In E. Perecman (Ed.), (pp. 41-72). New York: IRBN Press.

Prabhakaran, V., Narayanan, K., Zhao, Z., \& Gabrieli, J. D. (2000). Integration of diverse information in working memory within the frontal lobe. Nature Neuroscience, 3, 85-90. 
Riggs, K. J., McTaggart, J., Simpson, A., \& Freeman, R. P. (2006). Changes in the capacity of visual working memory in 5- to 10-year-olds. Journal of Experimental Child Psychology, 95, 18-26.

Roodenrys, S., Hulme, C., \& Brown, G. (1993). The development of short-term memory span: separable effects of speech rate and long-term memory. Journal of Experimental Child Psychology, 56, 431442.

Rosen, V. M., \& Engle, R. W. (1997). The role of working memory capacity in retrieval. Journal of Experimental Psychology-General, 126, 211-227.

Rosler, F., Heil, M., \& Roder, B. (1997). Slow negative brain potentials as reflections of specific modular resources of cognition. Biological psychology, 45, 109-141.

Ross, G. S. (1980). Categorization in 1- to 2-year-olds. Developmental Psychology, 16, 391-396.

Rypma, B., Prabhakaran, V., Desmond, J. E., Glover, G. H., \& Gabrieli, J. D. (1999). Load-dependent roles of frontal brain regions in the maintenance of working memory. Neuroimage, 9, 216-226.

Savage, C. R., Deckersbach, T., Heckers, S., Wagner, A. D., Schacter, D. L., Alpert, N. M., et al. (2001). Prefrontal regions supporting spontaneous and directed application of verbal learning strategies: evidence from PET. Brain : a journal of neurology, 124, 219-231.

Schelbe, J. L., Therriault, D. J., \& Miller, M. D. (2012). Classifying retrieval strategies as a function of working memory. Memory \& Cognition, 40, 218-230.

Schneider, W., Kron, V., Hunnerkopf, M., \& Krajewski, K. (2004). The development of young children's memory strategies: First findings from the Wurzburg Longitudinal Memory Study. Journal of Experimental Child Psychology, 88, 193-209.

Schneider, W., \& Pressley, M. (1997). Memory development between two and twenty (2nd ed.). Mahwah, New Jersey: Lawrence Erlbaum Associates.

Schneider, W., \& Pressley, M. (2013). Introduction to memory development during childhood and adolescence. Mahwah, New Jersey: Taylor \& Francis.

Siegel, L. S. (1994). Working-Memory and Reading - a Life-Span Perspective. International Journal of Behavioral Development, 17, 109-124.

Smith, E. E., \& Jonides, J. (1999). Storage and executive processes in the frontal lobes. Science, 283, 16571661.

St Clair-Thompson, H., Stevens, R., Hunt, A., \& Bolder, E. (2010). Improving children's working memory and classroom performance. Educational Psychology 30, 203-219.

St Clair-Thompson, H. L., \& Gathercole, S. E. (2006). Executive functions and achievements in school: Shifting, updating, inhibition, and working memory. Quarterly Journal of Experimental Psychology, 59, 745-759.

Stroop, J. R. (1935). Studies of interference in serial verbal reactions. Journal of Experimental Psychology, 18, 643-662.

Swanson, H. L., Howard, C. B., \& Saez, L. (2006). Do different components of working memory underlie different subgroups of reading disabilities? Journal of learning disabilities, 39, 252-269.

Tulving, E. (1972). Episodic and semantic memory. In E. Tulving \& W. Donaldson (Eds.), Organization of memory (pp. 381-403). New York: Academic Press.

Turley-Ames, K. J., \& Whitfield, M. M. (2003). Strategy training and working memory task performance. Journal of Memory and Language, 49, 446-468.

Unsworth, N., Brewer, G. A., \& Spillers, G. J. (2013). Working memory capacity and retrieval from longterm memory: the role of controlled search. Memory \& Cognition, 41, 242-254.

Wechsler, D. (1991). Wechsler Intelligence Scale for Children-Third Edition. New York: Psychological Corporation.

Wendelken, C., Bunge, S. A., \& Carter, C. S. (2008). Maintaining structured information: an investigation into functions of parietal and lateral prefrontal cortices. Neuropsychologia, 46, 665-678. 


\section{Chapter 2}

\section{The development of non-spatial working memory capacity during childhood and adolescence and the role of interference control; an N-Back task study}

Corresponding publication:

Schleepen, T.M.J., \& Jonkman, L.M. (2010). The development of non-spatial working memory capacity during childhood and adolescence and the role of interference control; an N-Back task study. Developmental Neuropsychology, 35, 37-56. 


\begin{abstract}
To investigate the role of interference control on the development of working memory (WM) capacity, 6-12 year-old children and adults performed an N-Back task with differing WM-load and interference control demands. Correlation analyses between flanker interference scores and WM-load levels showed that interference control was only required in the 2-back condition. While WM maintenance (1-back task) reached adult accuracy levels at age 10-12, the ability to maintain information in WM during distraction (2-back-task) displayed protracted maturation into adolescence. This is suggested to reflect yet immature connections between prefrontal and posterior association areas, respectively involved in interference control and WM storage.
\end{abstract}




\section{Introduction}

The ability to maintain and manipulate information in working memory (WM) allows us to perform well in a variety of cognitive tasks and to function effectively in everyday life. In children as well, effective use of this capability is important for successful cognitive development and academic achievement, since WM capacity plays an important role in learning of complex cognitive skills, like language, mathematics or reasoning (Pickering, 2006).

According to the influential WM model of Baddeley and Hitch (1974), WM consists of a central executive that acts as a supervisory system and is closely linked to two domain-specific buffers, respectively involved in the processing and short-term storage of nonspatial (verbal) or spatial information (Baddeley, 1992). Research has consistently shown that maintenance and manipulation of information in these respective systems is more difficult for children than for adults (Baddeley, 1992; Gathercole, 1998; Gathercole, 1999). However, there is inconsistency in the ages at which mature WM function is reported in different studies. Such inconsistencies might be explained by the large differences in experimental tasks that are used to determine WM ability. For instance, different developmental trajectories have been reported for spatial or non-spatial WM (Isaacs, 1989; Nichelli, Bulgheroni, \& Riva, 2001; Pickering, 2006; Van Leijenhorst, Crone, \& Van der Molen, 2007). Furthermore, in many WM tasks used in the developmental literature, participants are required to perform activities that depend upon abilities (e.g. reading or arithmetic skills) that also undergo important development during childhood, and thus might confound WM results (Towse, Hitch, \& Hutton, 2002). Reliance on such higher-order skills also complicates inclusion of younger children that do not yet master them. Finally, different developmental trajectories have been reported for performance in simple span (Riggs, McTaggart, Simpson, \& Freeman, 2006) and more complex WM tasks that, besides maintenance, also require manipulation of to-be stored information.

More specifically, while performance in simple span tasks has been shown to reach maturity during childhood, complex WM skills show protracted development into adolescence (for review see Bunge \& Wright, 2007). Recent developmental neuro-imaging studies have shown that such delayed development goes along with immaturity in frontal-parietal networks that underlie mature complex WM skills. Crone, Wendelken, Donohue, van Leijenhorst and Bunge (2006) showed that only in a complex WM task requiring manipulation, 8-12 year-old children showed immature performance and failed to activate the same WM-related brain areas as adults (e.g. the Dorsolateral Prefrontal Cortex (DLPFC) and superior parietal cortex). In a study by Scherf, Sweeney and Luna (2006) this reliance of children (1013 years) on ventromedial prefrontal cortex, instead of on core WM regions such as DLPFC and parietal cortex, was confirmed in a visuo-spatial WM task. Interest- 
ingly, comparable fronto-parietal networks have been implicated in late development of executive control processes, such as response inhibition or interference control, that also continues into adolescence (Bunge \& Wright, 2007; Casey et al., 1995; Diamond, 2002; Huizinga, Dolan, \& van der Molen, 2006; Kanemura, Aihara, Aoki, Araki, \& Nakazawa, 2003; Luna \& Sweeney, 2004; Sowell et al., 1999). This raises the important question of how much the development of complex WM skills depend on immaturity of executive control processes. Surprisingly, to our knowledge, the development of such specific WM-executive control interactions has not yet been directly studied. The aim of the present study is to investigate the role of one specific executive control process, namely interference control, on the development of WM capacity.

The present study focuses specifically on interference control because several lines of research have shown that the ability to suppress or ignore distracting information while holding information in memory plays an important role in the determination of one's WM capacity or the success of recall in WM tasks (de Fockert, Rees, Frith, \& Lavie, 2001; Lavie, Hirst, de Fockert, \& Viding, 2004; McNab \& Klingberg, 2008; Sakai, Rowe, \& Passingham, 2002). Furthermore, multiple studies have shown that individuals with low WM-capacity are more prone to distraction in attention tasks or display more attention problems. This has been demonstrated for adults (Kane \& Engle, 2003) as well as children (Gathercole et al., 2008). These results all demonstrate that interference control and WM capacity are highly dependent upon each other, as is supported by their reliance on overlapping brain circuitry, including lateral prefrontal cortex (PFC) (Chao \& Knight, 1995; D'Esposito, Postle, Jonides, \& Smith, 1999; Postle, 2005).

An experimental task that seems suitable to investigate the role of interference control ability on the development of WM capacity in simple and complex WM tasks, is the N-Back task. In this task, WM load is parametrically varied such that the effect of load on reaction time (RT) and accuracy rates can be parceled out. In a typical N-Back task, sequences of items (either spatial or non-spatial) are shown and participants are instructed to respond to items that are identical to the item presented one, two or more trials back in the sequence. Because this task requires to keep in mind the $n$ th-item back in the sequence, a greater information load has to be stored in WM with increasing N-Back load. Furthermore, previous work suggests that with increasing $n$ in the N-Back task, increased demands are posed on executive control processes. Smith and Jonides (1997) compared PET activations in 0,1, 2 and 3-back conditions in a verbal task and showed that the number of activated brain areas increased with $n$, but DLPFC only became involved in the task starting from the 2-back condition. Because of reported links between DLPFC activation and executive control processes (e.g., D'Esposito et al., 1995; Diamond, 2002), this finding implies that executive control is particularly involved, or at least more involved, in 2-(or higher) back conditions, when compared to 1- 
back. Supportive evidence for the involvement of interference control processes in 2-back tasks comes from a developmental fMRI study of Ciesielski, Grant, and Ahlfors (2006). In this study, a correlation of $r=.50$ between performance in a categorical 2-back task and a Stroop task was reported. However, no comparison was made between 0,1 , and 2-back levels, so that it was not demonstrated that this correlation was specific for the 2-back condition.

Thus, the N-Back task seems very well suited to on the one hand study the development of WM capacity by parametrically varying load, while on the other hand determining how demands on executive control functions influence such patterns. Nevertheless, to our knowledge, there are only a few studies that have used the N-Back task to study the development of working memory (Kwon, Reiss, \& Menon, 2002; Vuontela et al., 2003). These studies only used visuo-spatial tasks and did not report on developmental differences between 1 and 2-back tasks that both require maintenance but differ in executive control demands (see Smith \& Jonides, 1997). Furthermore, Vuontela et al. (2003) did not include an adult age group, missing potential continued development during adolescence, an important period for executive control development (Luna \& Sweeney, 2004). One recent developmental study by Conklin, Luciana, Hooper, and Yarger (2007), did examine the role of distinct executive processing requirements on WM development in children and adolescents (9-17 years). Delayed development of verbal and nonverbal WM performance was reported when the tasks required high-executive control. However, in this study WM tasks mutually varied in nature and type of required executive control. Parametric manipulation of WM-capacity (Load) and interference control within one task is important to exclude the influence of other (perceptual or motor) task demands on developmental patterns.

\section{The present study}

In sum, developmental studies of non-spatial WM employing the N-Back task are scarce and only one study included children, adolescents and adults (but starting from age 9). Furthermore, no study so far directly investigated the impact of differences in required executive control on the development of WM performance in tasks in which WM-capacity (load) is parametrically varied. The present study aims to fill this void by investigating the development of WM-capacity and interference control interactions from young childhood to adulthood, by comparing performance across N-Back load levels that differ in executive control. The current study will use a verbal version of the N-Back task (consisting of 0, 1 and 2 back conditions) because, besides that relatively little is known about age-related changes in verbal N-Back task performance, proof for the involvement of executive control in 2-back and higher came from a similar verbal task (Smith \& Jonides, 1997). 
It is hypothesized that performance in the 2-back condition (demanding high levels of executive control) will show protracted maturation into adolescence, since the development of executive control is known to depend heavily on the development of prefrontal cortex which is not anatomically and functionally mature until late adolescence (Giedd et al., 1999; Gogtay et al., 2004; Pandya, 1987). In contrast, performance in the 1-back task, requiring mainly maintenance of information in WM, is hypothesized to reach maturity before adolescence. To confirm the (enhanced) involvement of executive control processes particularly in the 2-back task, all subjects also performed a flanker task (Eriksen \& Eriksen, 1974) that measures the executive process of interference control. Interference control is thought to be involved in the 2-back task since, at each trial, subjects have to update WM with a new letter while in the meantime suppressing the influence of this letter on the current response decision. It is hypothesized that in case of (higher) involvement of interference control processes in the 2-back task, there will be a positive correlation between the size of RT-interference effects in the flanker task and the size of performance decrements in the 2-back (vs. the 1-back) task.

\section{Method}

Subjects

Three groups of children $(n=57)$ and one group of adults $(n=21)$ participated in the present study. Demographic information (age, gender, IQ scores, Socioeconomic Status (SES), attention scores and group size) for all groups is shown in Table 1. The choice of age groups is based on previous studies showing important developmental transitions in either inhibition or interference control during these age periods (Jongen \& Jonkman, 2008; Jonkman, 2006).

Exclusion criteria for both children and adults were 1) presence of neurological or psychiatric disorders 2) medication use 3) a score in the clinical range on relevant attention rating scales (see measures paragraph below) and 4) an IQ below 80. One child in the 10-12 years-old group was excluded from the study because of scores more than 3 standard deviations from the mean for both hit and false alarm percentages. All children were recruited from two different local primary schools and received a present for participation. Adults were university students of Maastricht University and received either course credit or were paid for participation. Written informed consent was obtained from both the adult subjects and the parents of the children. The study was approved by a local ethical committee at the Faculty of Psychology and Neuroscience, Maastricht University. 
Table 1. Demographic characteristics per age group.

\begin{tabular}{|c|c|c|c|c|}
\hline \multirow[b]{2}{*}{ Variable } & \multicolumn{3}{|c|}{ Age Group } & \multirow[b]{2}{*}{ Adults (19-28 years) } \\
\hline & $6-7$ & $8-9$ & $10-12$ & \\
\hline$N$ & 19 & 21 & 17 & 21 \\
\hline Gender ( $\%$ female) & 47 & 48 & 65 & 52 \\
\hline \multicolumn{5}{|l|}{ Age (in years) } \\
\hline$M$ & 7 & 9 & 11.2 & 21.7 \\
\hline$S D$ & 0.52 & 0.62 & 0.81 & 2.70 \\
\hline \multicolumn{5}{|l|}{ Attention score ${ }^{\mathrm{a}}$} \\
\hline$M$ & 52.8 & 55.8 & 53.8 & 52.2 \\
\hline$S D$ & 5.5 & 5.8 & 5.3 & 5.0 \\
\hline range & $50-70$ & $50-67$ & $50-66$ & $43-60$ \\
\hline \multicolumn{5}{|l|}{ Estimated $\mathrm{IQ}^{\mathrm{b}}$} \\
\hline$M$ & 111.7 & 104.9 & 102.9 & 117.1 \\
\hline$S D$ & 10.2 & 12.2 & 12.4 & 10.4 \\
\hline range & $91-132$ & $88-132$ & $80-123$ & $100-143$ \\
\hline \multicolumn{5}{|l|}{ SESc } \\
\hline$M$ & 5.4 & 5.6 & 5.4 & - \\
\hline$S D$ & 1.7 & 1.5 & 1.4 & - \\
\hline
\end{tabular}

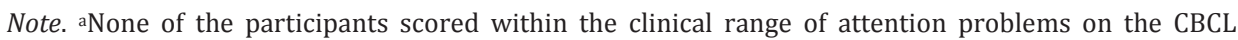
(children) or ACTeRS (adults). ' IQ scores differed significantly between the groups, $F(3,74)=6.43, \mathrm{P}$ $<$.01.Bonferonni-corrected post hoc comparisons revealed that both 8-9 and 10-12-year-olds differed significantly from the group of adults $(p<.01)$. Therefore, all analyses were performed with IQ as covariate, which revealed no altered significant levels and thus all further analyses were performed without IQ as covariate. cSocioeconomic level (SES) was determined by Hollingshead (1975) occupational scale for the parent holding the higher status job ( 1 or $2=$ unskilled or unemployed positions, 3 or $4=$ skilled or semiskilled laborers, 5 or $6=$ managerial professions, 8 or $9=$ major professions.). Parental occupation data were not available for one child in the group of 6-7 and two children in the group of 8-9 yearolds. SES was not available for adults, but all adults were university students.

\section{Measures}

Attention rating scales. Whereas one of the exclusion criteria was a clinical diagnosis of ADHD, all children and adults were additionally screened for attention deficits. In the case of children parents were asked to fill out the Child Behavior Checklist (CBCL- 4-18 years; Achenbach, 1991). Adults were screened for the presence of inattention symptoms by filling out the ACTeRS self-report form (Ullman, Sleator, \& Spraque, 1991). This self-report form consists of 35 items, of which 10 items form the attention-subscale. For the CBCL, T-scores between 67-70 are considered as borderline-clinical and a T-score $>70$ is considered a clinical score. Two children scored within the subclinical range (i.e. one in the 6-7 and one in the 8-9 group). However, these children were not excluded from the present study because their performance in the N-Back task was not deviating from that of the 
mean group. The ACTeRs raw scores are converted to gender neutral percentile ranks and T-scores, with a lower score indicating enhanced problem behavior; a Tscore of 46 or higher on the Attention subscale indicates a score within the $70 \%$ range of the population scores (according to the manual, subjects diagnosed with ADHD scored in the top $10 \%$ of the population range, corresponding to a $\mathrm{T}$-score below 41 on this scale).

Intelligence. In order to assess IQ, subjects were administered two subtests (i.e. vocabulary and block design) of the Wechsler Intelligence Scale for Children (WISC-III, Dutch version) and the Wechsler Adult Intelligence Scale (WAIS-III, Dutch version). The estimated IQ score on basis of these two subtests has a mean reliability and validity of 9 (Jeyakumar, Warinner, Raval, \& Ahmad, 2004; Spreen, 1998), when compared to the IQ derived after completion of the full test.

\section{Experimental computer tasks}

$N$-Back task. In the present study a letter version of the $\mathrm{N}$-Back task was used in which semi-random sequences of letters (A, B, F, G, H, K, L, S, T, W, X, Z) appeared one at a time at the centre of the screen (see Figure 1). The letters (height: $1 \mathrm{~cm}$, width: $0.5 \mathrm{~cm}$ ) were green and presented between two white vertical bars (height: $1.5 \mathrm{~cm}$ ) on a black background. Subjects were seated at a distance of approximately $50 \mathrm{~cm}$ in front of a 17 inch VGA monitor. Stimulus presentation and acquisition was controlled by ERTS-software. The N-Back task consisted of 0-, 1- and 2-back conditions. Participants were instructed to press a response button with their right index finger, whenever they detected a target event. In 1- and 2-back conditions a target was defined as a letter that was identical to the letter presented 1 (e.g. T-T) or 2 (e.g. A-B-A) trials back in the sequence. In the 0-back condition, the target event was the appearance of the letter " $\mathrm{X}$ ".

The task consisted of a total number of 360 trials, which were presented in six blocks of 60 stimuli, two blocks per condition (0,1,2-back). The order of block presentation was randomized with the restriction that each condition was presented within the first three blocks. There were 3 versions of the task differing in the order of presentation of 0,1 and 2-back conditions; version 1: $0,2,1-2,1,0$ (back), version 2: 0, 1, 2 - 1, 0, 2 (back) and version 3: 2, 0, 1 - 1, 2, 0 (back). Versions were presented in a counterbalanced order between participants, within groups. To test whether there were differences in performance between groups due to order of task presentation, this variable was included as a covariate in all analyses and did not change results. All task blocks had a similar target frequency of $33.3 \%$, they only differed with respect to the appearance of the letter $\mathrm{X}$ in the 0 back being 33.3\%, but not in the other two conditions. Each trial lasted $2000 \mathrm{~ms}$ with a stimulus duration of $500 \mathrm{~ms}$ and a fixed interstimulus-interval (ISI) of 1500 ms. 


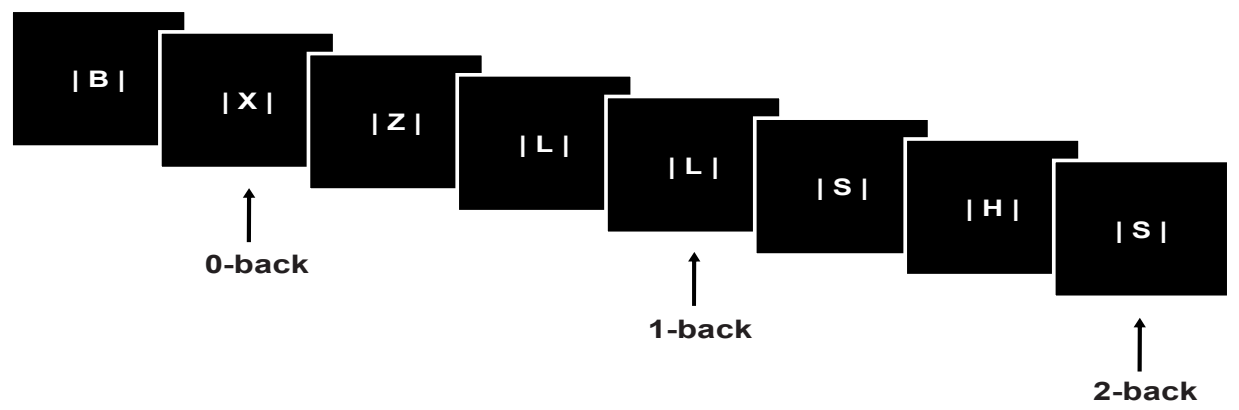

Figure 1. Illustration of the N-Back task with examples of $0-, 1-$, and 2-back conditions

Flanker task. A letter version of the flanker task was used to provide a measure of response interference effects (Eriksen \& Eriksen, 1974). Subjects were presented with stimuli consisting of arrays of three letters (e.g. B B B) that were presented in the middle of the computer screen The task instruction was to press a button in response to a centrally presented target letter, while ignoring the two flanking letters. The target letters $\mathrm{B}$ and $\mathrm{H}$ were associated with a right index finger response and the target letters $\mathrm{F}$ or $\mathrm{T}$ with a left index finger response (this was counterbalanced between subjects, within groups). The two flanking letters were presented at the left and the right of the central target letter and were always identical to each other. Response interference effects were determined by the difference in reaction time (RT) between congruent trials (i.e. the target letter was similar to the flanking letters; e.g. F F F) and incongruent trials (the target letter was surrounded by different letters that activate a response that is in conflict with the target response). A total of 320 trials were presented in four blocks consisting of 80 trials. Each trial lasted 2500 ms, with a stimulus duration of $1000 \mathrm{~ms}$ and an inter-stimulus interval of $1500 \mathrm{~ms}$.

\section{Procedure}

Adults were tested in one experimental session that lasted approximately 3 hours. After informed consent was obtained from adults, they filled in the ACTeRS selfreport form. The $\mathrm{N}$-Back task was administered as the last task in a series of tasks that were part of a larger developmental study that included measurement of EEG, from which the data will be presented elsewhere. One of the tasks that was used in these EEG measurements was the Flanker task. The complete behavioral and ERP results of this task go beyond the present study aims and will be presented elsewhere. For the purpose of verifying whether interference control was particularly involved in the 2-back condition, in the present study only part of the behavioral (RT) data from the flanker task were used for the correlation analyses (see follow- 
ing section). Before the N-Back task, a digit span backward, adapted from the WISC-III, Dutch version (Wechsler, 1991) and the two IQ subtests were administered. The digit-span backward raw scores were collected as additional individual measures of "complex" WM capacity (requiring maintenance and manipulation skills; see following section for how this score was used in the correlation analyses). Children were administered the same tasks as adults, but they were tested in two separate experimental sessions in a quiet room at their school. While the EEG tasks were administered during the first session (lasting about 2 hours), children had to complete the behavorial tasks (including the N-back task) during the second session (lasting about 1 hour).

All experimental tasks were practiced until participants reached a predetermined performance criterion (75\% correct responses). This criterion was used to verify that participants were able to perform the task above chance level before the experimental task was started. In the practice task of the N-Back task, subjects practiced each of the three conditions (0,1 and 2-back) that were administered in three blocks of 25 trials each. During these practice blocks, each trial was provided with feedback: correct in case participants correctly identified the target, false whenever participants responded to a non-target and forgotten when participants did not react before the end of the trial. In the experimental task, no feedback was provided anymore.

\section{Data analysis}

Mean reaction times to correctly identified targets (RT), mean percentage of correctly identified targets (hit \%), mean percentage of false alarms (defined as responses to non-targets) and the signal-detection parameter d-prime $(d$ ') were computed in 0-, 1- and 2-back conditions. To test for developmental trends in performance in the N-Back task depending on WM load, repeated measures analysis of variance was performed entering the between subjects factor Group (4 groups, children aged 6-7, 8-9, 10-12 and adults) and the within subjects factor WM load $(0-, 1$ - and 2-back). Separate analyses were performed for the dependent variables RT, \% hits, \% false alarms and $d^{\prime}$. Whenever a significant Group (4) x Load (3) interaction was found, two planned ANOVA's with a factor Group (4 groups) and Load (now consisting of two load levels) were carried out to examine developmental differences in Load effects separately for 0 vs. 1-back and 1- vs. 2-back. In case of significant Group x Load 0 vs. 1-back or Group x Load 1 vs. 2-back interactions, further post-hoc group comparisons were performed.

In addition to the ANOVA tests and post-hoc between group comparisons, polynomial contrast analysis was performed including the Group factor to study developmental trends in the data across all four age groups in 0, 1 and 2-back conditions. Because the present study included four age groups, linear, quadratic and 
cubic trends were explored for all N-Back levels and for the separate dependent measures. A significant linear trend would indicate a linear increase of performance across age, whereas significant quadratic and cubic contrasts would signify non-linear developmental patterns. For all statistical analyses a two-tailed significance level of $p<.05$ was adopted.

To assess the assumed differential involvement of interference control processes in the different $\mathrm{N}$-Back conditions, the \% hit difference in 0 - vs. 1 - and 1 - vs. 2-back conditions was correlated with RT-interference effects as obtained in the flanker task. To investigate the involvement of WM maintenance and manipulation processes in the different N-Back task conditions, correlations were also computed between the individual raw scores on the digit span backward task and the accuracy (hit \%) decrement in 0- vs. 1- and 1- vs. 2-back tasks.

\section{Results}

Mean RT, \% hits, \% false alarms and d' (and SD's) in 0, 1, and 2-back conditions are presented in Table 2. To enhance readability of the results section, statistical (F and p) values for Group x Load interactions as well as for post-hoc group comparisons are shown in Table 3.

\section{Reaction times}

A significant Group x Load interaction was found $(p<.0001)$ when comparing reaction times in 0-, 1- and 2-back conditions. This interaction effect was followed up by two planned contrasts examining Group x Load interactions respectively comparing 0 and 1-back and 1 and 2-back tasks. This analysis showed a significant Group x Load interaction when comparing 0 and 1-back conditions $(p<.00001)$ (maintenance). Further group comparisons showed significantly smaller load effects on RT in adults than in 10-12, 8-9 and 6-7 year-olds (all $p s<.01$ ). In turn, 1012 year-old children had smaller 1 -back load effects on RT than $8-9(p<.05)$ and 67 year-olds $(p<.0001)$.

The planned ANOVA investigating effects of age on Load effects between 1 and 2-back conditions (maintenance + interference control) yielded significant main Load, $F(1,74)=50.1, p<.00001, \eta 2 p=.40$, and Group effects, $F(3,74)=33.8$, $p<.00001, \eta 2 p=.58$, indicating that Load effects were present equally strong in all age groups, and irrespective of load there was a decrease in RT across childhood and adolescence.

The polynomial trend analysis yielded significant linear effects (and nonsignificant quadratic or cubic effects) for the factor Group in all N-Back conditions 
$(p<.0001)$. These effects reveal a non-specific linear decrease in RT across childhood and adolescence into adulthood (see Figure 2).

\section{Percentage of hits}

For \% hits, a significant Group x Load interaction was found $(p<.00001)$ when all load levels were included. The planned ANOVA investigating effects of age on Load effects between 0 and 1-back conditions yielded a significant Group x Load interaction $(p<.00001)$. Further group comparisons showed smaller Load effects in 10-12 year-olds than in adults $(p<.05)$, but both groups had smaller Load effects than 8-9 $(p<.0001$ and $p<.01$, respectively) and 6-7 year-old children $(p<.00001$ and $p<.0001$, respectively).

The planned ANOVA investigating effects of age on Load effects between 1 and 2-back conditions also yielded a significant Group x Load interaction ( $p>$.00001). Further group comparisons showed that adults had significantly lower decreases in accuracy from the 1 to the 2-back condition than all children groups (all $p s<.0001)$. Furthermore, the 10-12 year-olds had a smaller Load effect than 8-9 $(p<.05)$ and 6-7 year-olds ( $p=.05)$ (see Table 3 ).

These patterns were supported by polynomial trend analyses that tested for linearity or nonlinearity of Group effects in the 0-, 1- and 2-back conditions separately. In the 0 -back condition a significant linear trend for the factor Group was found, $F(1,74)=12.1, p<.01$, indicating a linear increase in percentage of hits with age (however, as can be seen in Figure 1, percentages of hits were almost at ceiling level in the 0-back condition). A quadratic Group effect was found in the 1-back condition, $F(1,74)=8.1, p<.01$, revealing an increase in percentage of hits during childhood years reaching a plateau at age 10-12, and displaying no (or minimal) accuracy differences between 10-12 year-olds and adults. As opposed to in the 1back condition, a significant linear trend for the Group factor was found for the 2back condition, $F(1,74)=86.5, p<.00001$, indicating a steady increase in the percentage of hits across childhood and adolescence into young adulthood (see Figure 2). These quadratic (1-back) and linear (2-back) trends (and the ANOVA results) supported the hypotheses of early maturation of relatively simple WM skills, such as maintenance in the 1-back task, and protracted development of active WM maintenance during interference in the 2-back task.

\section{Percentage of false alarms}

A significant Group x Load interaction was found $(p<.01)$ when comparing the three load levels. Further planned ANOVA analysis yielded a significant Group x Load interaction when comparing 0 - and 1-back conditions $(p<.005)$. Further group comparisons indicated significantly smaller (or absent) increases in false 
alarms in the 1-back condition (compared to 0-back) in adults than in 10-12, 8-9 and 6-7 year-old children (all $p s<.05$ ). Between the children groups, there was a significantly larger increase in false alarms in the 1-back (vs. 0-back) condition in 6-7 than 8-9 year-olds ( $p<.05$ ), but not between age 8-9 and 10-12 (see Table 3).

Table 2. Mean reaction times (RT), mean percentage of hits, mean percentage of false Alarms and mean d-prime (d') scores (Standard Deviations in Parentheses) per N-Back load per age group.

\begin{tabular}{lccc}
\hline & \multicolumn{3}{c}{ WM-Load } \\
\cline { 2 - 4 } RT (ms) & 0 & 1 & \\
$6-7$ & $613(94)$ & $816(172)$ & $865(151)$ \\
$8-9$ & $539(91)$ & $675(147)$ & $787(154)$ \\
$10-12$ & $469(71)$ & $541(93)$ & $650(157)$ \\
adults & $392(49)$ & $428(63)$ & $514(112)$ \\
Hits (\%) & & & \\
$6-7$ & $98(3.0)$ & $83(10.5)$ & $55(20.4)$ \\
$8-9$ & $99(1.8)$ & $90(7.2)$ & $63(12.9)$ \\
$10-12$ & $100(1.3)$ & $98(1.9)$ & $80(12.4)$ \\
adults & $100(0.5)$ & $96(3.9)$ & $92(6.4)$ \\
False alarms (\%) & & \\
$6-7$ & $6.05(5.55)$ & $14.1(15.8)$ & $22.6(19.2)$ \\
$8-9$ & $3.21(3.38)$ & $5.48(3.76)$ & $15.6(6.80)$ \\
$10-12$ & $2.50(2.50)$ & $5.29(4.83)$ & $16.2(7.34)$ \\
adults & $0.95(1.47)$ & $0.71(2.26)$ & $5.2(5.2)$ \\
Memory recognition: d' & & & \\
$6-7$ & $4.18(.70)$ & $2.67(.73)$ & $1.34(.65)$ \\
$8-9$ & $4.75(.46)$ & $3.4(.53)$ & $1.68(.39)$ \\
$10-12$ & $4.83(.47)$ & $4.28(.60)$ & $2.33(.58)$ \\
adults & $5.1(.25)$ & $4.42(.43)$ & $3.64(.86)$ \\
\hline & & &
\end{tabular}

The planned ANOVA including the 1-vs. 2-back contrast yielded significant main effects of the factors Load, $F(1,74)=69.1, p<.0001, \eta 2 p=.48$, and Group, $F(3,74)=$ $10.4, p<.0001, \eta 2 p=.29$, but no interaction. The main Load effect implies that there were significant and comparable increases in false alarms in the 2-back task (compared to 1-back) in all age groups. The main group effect reflects a general decrease in false alarms from young childhood to adulthood that is independent of WM-load (see Figure 2).

Trend analyses showed significant linear trends for the factor Group in the 0back, $F(1,74)=20.4, p<.0001,1$-back, $F(1,74)=22.8, p<.00001$, and 2-back condi- 
tions, $F(1,74)=21.8, p<.0001$, indicating linear decreases in false alarms from young childhood to adulthood in all task conditions. 


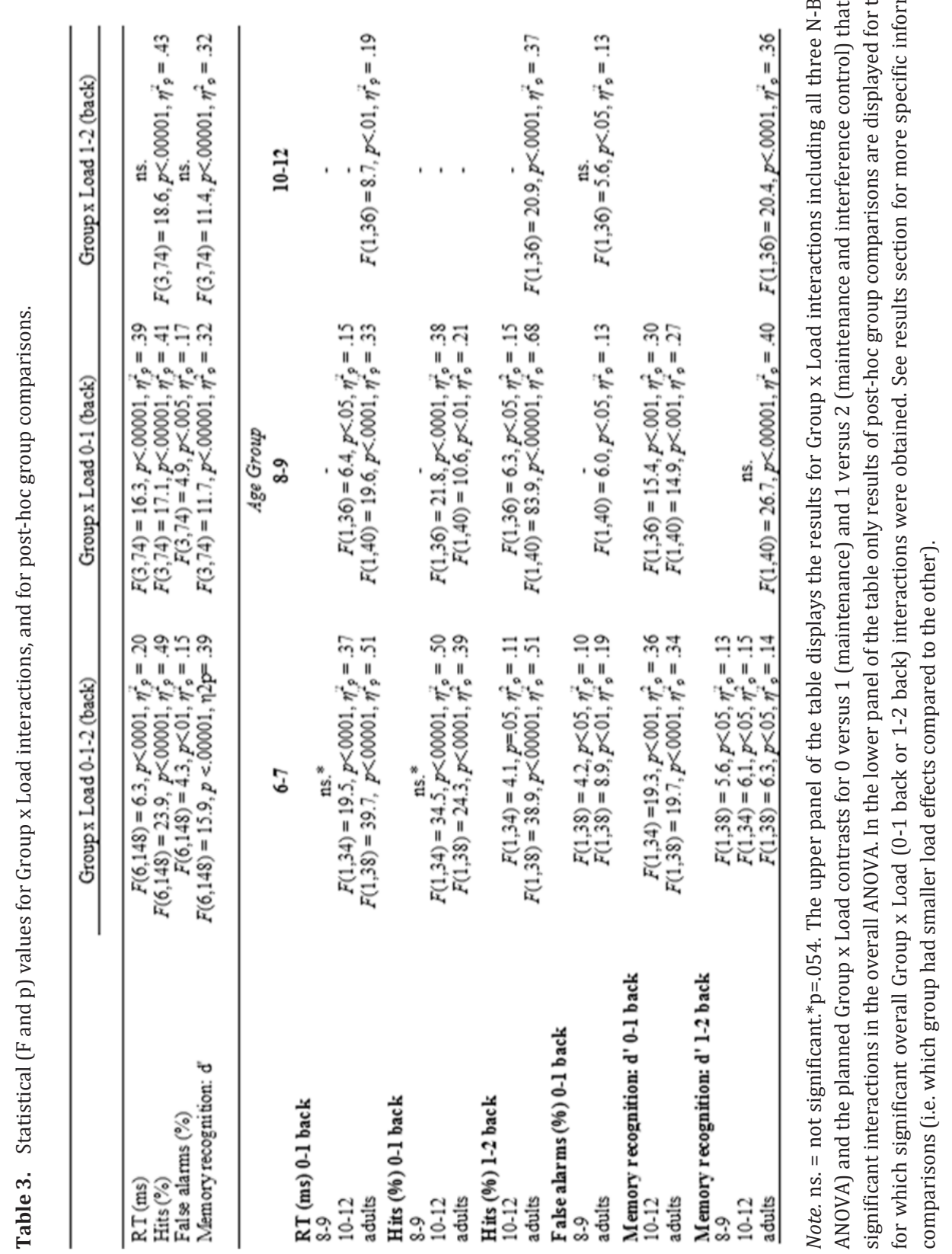


To investigate developmental differences in the perceptual sensitivity to discriminate letters that had or had not been presented as WM-probes, the signal detection parameter $d^{\prime}$ (that takes into account both the proportion of hits and false alarms) was calculated according to Boice and Gardner (1988). The overall ANOVA including all three load levels yielded a significant Group x Load interaction $(p<.00001)$. Planned follow-up analyses showed highly significant Group x Load interactions comparing the 0 - and 1 back conditions and the 1- and 2-back conditions (both $p s<.00001$ ). Group comparisons for 0 vs. 1-back showed that adults and 10-12 year-olds had comparable load effects on $d^{\prime}$, that were smaller than those of 6-9 year-olds (all $p s<.00001$ ). Load effects did not differ between 6-7 and 8-9 yearolds. Group comparisons for 1 vs. 2-back showed that Load effects on $d$ ' were significantly smaller in adults than in 8-12 ( $p<.00001)$ and 6-7 year-olds children $(p<.05)$. Furthermore, 8-12 year-old children had smaller load effects than 6-7 year-olds $(p<.05)$.

These patterns were confirmed by trend analyses that revealed a linear Group effect in the 0-back task, $F(1,74)=33.4, p<.00001)$, and quadratic Group effects in 1-back, $F(1,74)=5.12, p<.05)$, and 2-back, $F(1,74)=10.8, p<.005$, tasks (see Figure 2 ). The quadratic Group effect in the 1-back task revealed an increase in target/non-target discrimination during childhood years reaching a plateau at age 1012. In the 2-back task, there was an increase in discrimination ability across age, but the quadratic effect showed that the steepest increase in $d^{\prime}$ took place after age 10-12 (see Figure 2). These results confirm the hypotheses of early maturation of maintenance in the 1-back task, and protracted development of active WM maintenance during interference the 2-back task. 


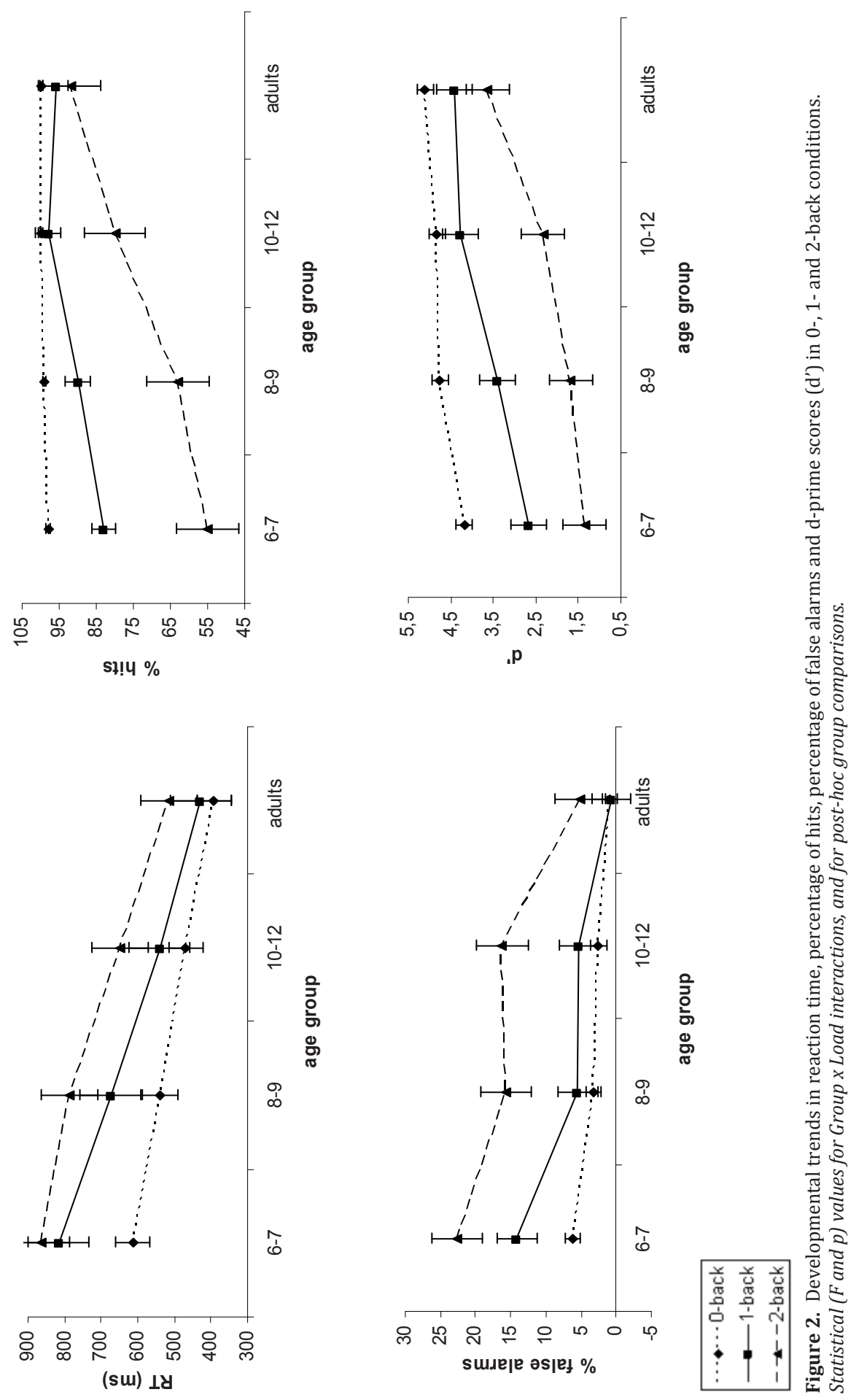




\section{Correlation analyses}

To assess the assumed involvement of interference control processes in especially the 2-back condition, pearson's correlations were computed between the \% hit difference in 0 vs. 1-back and 1 vs. 2-back conditions and response interference effects (i.e. RT difference between congruent vs. incongruent trials) as obtained in the parallel performed flanker task. Supporting the hypothesis, a positive correlation between the difference in \% hits between 2- and 1-back conditions and the flanker interference effect was found, $r=.29, p=.008$. This positive correlation indicates that subjects that showed the strongest performance decrease from 1- to 2-back, had worse interference control (largest interference effects) in the flanker task. As hypothesized, the \% hit difference between 0 - and 1-back conditions did not correlate significantly with flanker interference effects, $r=.02, p=.89$.

Examining the correlation between performance on WISC-III (Dutch version) backward counting (as an index of WM updating and manipulation) and performance on the N-Back task, pearson's correlations were also computed between differences in hit \% in 0 vs. 1 and 1 vs. 2 back conditions and raw backward counting scores. While significant negative correlations were found for 0 vs. $1, r=-.37$, $p=.001$, and for 1 vs. 2-back and backward counting, $r=-.49, p=.0001$, the correlation was higher for the 1-2-back comparison. The absence of a correlation between flanker interference effects and backward counting scores, $r=-.065, p=.56$, suggests that both are independent contributors to the accuracy decrease in the 2back condition. Note that the data were checked for outliers, but none were present.

\section{Discussion}

The purpose of the present study was to investigate the development of the ability to maintain information in WM in conditions differing in the amount of needed interference control. It was hypothesized that performance of WM tasks requiring relatively high levels of interference control would undergo delayed maturation into adolescence due to the protracted development of brain circuitry involved in exertion of executive control processes such as interference control (Casey, Giedd, \& Thomas, 2000; Giedd, et al., 1999; Gogtay, et al., 2004; Luciana \& Nelson, 1998; Pandya, 1987). To test these hypotheses, three groups of children of 6-7, 8-9 and 10-12 years and adults performed a non-spatial N-Back task with 0-, 1- and 2-back conditions. Whereas age-related changes in the 1- (relative to 0 -) back condition were assumed to reflect the development of WM maintenance, the development of 
WM maintenance during the simultaneous control for interference was inferred from age-related changes in the 2- (relative to 1 ) back condition.

\section{The involvement of interference control and manipulation in the N-Back task}

It was hypothesized that interference control processes were particularly involved in the 2-back task, since besides an increase in WM-load, the intervening, to-be maintained, letter was assumed to interfere with the current response decision. This hypothesis was supported by the positive $(r=.29)$ correlation between the size of the accuracy decrement from the 1 to the 2-back condition and the size of RT-interference effects in a parallel performed flanker task. This correlation was not found for the $\%$ hits decrement from the 0 - vs. the 1-back condition, indicating that cognitive control is not needed in relatively simple WM tasks that only require the maintenance of information, as was shown before by Stins, Polderman, Boomsma, and de Geus (2005). Such a correlation between accuracy of performance in the 2-back condition and interference control in children and adults was earlier reported by Ciesielski et al. (2006), but by comparing results across multiple load levels, the present data show that this correlation is specific for the 2-back condition. This finding is also in compliance with PET results from Smith and Jonides (1997) that showed activation of DLPFC, an area strongly associated with executive control, only in 2 and 3 -back, and not 1 or 0 -back conditions. Furthermore, significant negative correlations between accuracy decrements in 1 (vs. 0) and 2 (vs. 1)- back tasks and scores on a digit-span-backward task were found; the larger the performance decrements in 1- or 2-back tasks, the lower the WM-span scores on the digit span-backward task. This finding is interpreted as showing the involvement of WM maintenance and manipulation skills in both 1 and 2-back tasks. The higher correlation in the 2-back task might reflect relatively higher manipulation requirements in this task (Groeger, Field, \& Hammond, 1999). Finally, the absence of a correlation between digit-span backward scores and interference effects in the flanker task might indicate that WM maintenance and manipulation ability on the one hand and interference control ability on the other hand, contribute in an independent way to WM-performance in the 2-back task.

\section{The development of WM maintenance during low or high distraction}

First, main effects of the load manipulation (0-, 1-, 2-back) on memory accuracy (hits, false detections, $d^{\prime}$ ) and reaction time (RT) measures were present in all groups and showed a linear decrease (accuracy, $d^{\prime}$ ) or increase (RT) with increasing N-Back load. The decrease in hits and increase in false recognitions in the 2back relative to the 1-back task, is suggested to be caused by a disruption of the 
memory trace of the letter stored at the preceding trial due to the requirement to update WM with yet another letter.

With respect to development, supporting the hypotheses, for percentage correctly identified memory probes, a quadratic trend in the 1-back and a linear trend in the 2-back condition indicated different developmental patterns for WM maintenance in tasks that do or do not require simultaneous suppression of interfering information. While the quadratic trend in the 1-back task indicated that adult accuracy levels were reached at age 10-12, the linear trend in the 2-back task showed that WM accuracy continued to increase during adolescence when simultaneous interference control is required. This developmental pattern was supported by analyses of the signal detection parameter $d^{\prime}$ that takes into account both the proportion of true (hits) and false recognitions and thus reflects the perceptual sensitivity to discriminate between items that were or were not presented as memory probes (e.g. memory recognition). In the 1-back task, $d^{\prime}$ reached mature levels at age 10-12, whereas in the 2-back task, statistical analyses confirmed that the strongest improvement in memory recognition $\left(d^{\prime}\right)$ took place during adolescence (e.g. between age 10-12 and adulthood). This was for a large part caused by a strong (11\%) decrease in the number of false recognitions in the 2-back task after age 10-12. This decrease might well be related to frontal cortex development during adolescence since increased false recognitions have been reported in patients with damage to the frontal lobes and it has been suggested that the prevention of false memories requires PFC recruitment (for review see Schacter \& Slotnick, 2004). Finally, a comparable distinctive developmental pattern between 1 and 2-back tasks was not present when looking at speed of processing. Although reaction time increased considerably with $\mathrm{N}$-Back load, equally strong reductions in processing speed were seen during early childhood and adolescence in 0,1 and 2-back conditions. This is suggested to reflect a more global developmental increase in basic processing speed (Hale, 1990; Kail \& Park, 1992).

The delayed development of WM-accuracy and recognition memory into adolescence only in the 2-back task (which involves interference control), is in agreement with previous studies. These studies show that when children approach age 12 , they perform at adult accuracy levels in more simple WM storage tasks (Cowan, 1997), whereas in WM tasks requiring simultaneous higher-order cognitive control processes, mature levels are only reached in adolescence (Crone, et al., 2006; Diamond, 2002). Olesen, Macoveanu, Tegner, and Klingberg (2007) reported similar late development of WM accuracy in a spatial-WM task involving interference control. These authors showed that the WM performance of 13-year-old children was particularly less accurate than that of adults when maintenance was challenged by distraction. The present data support such late maturation of WMcapacity in tasks demanding interference control by parametrically varying WMload and executive control demands within one task in the same subjects, and us- 
ing subtractive logic to disentangle effects of load and executive control. Furthermore, the present study included 4 age groups, covering a much broader age range of 6-28 years. This delayed development of the ability to protect memory content from disruption when processing of potentially interfering information is required, is most likely due to protracted development of fronto-parietal brain circuitry underlying such a proficiency (Bunge \& Wright, 2007; Crone, et al., 2006; Olesen, et al., 2007). In particular, several developmental fMRI studies have reported that mature performance in tasks that involve relatively high levels of cognitive control goes along with increased recruitment and activation of PFC-parietal brain networks (Casey, et al., 1995; Crone, et al., 2006; Luna et al., 2001).

The exact mechanisms, by which the impact of distracting information on active WM maintenance is controlled is not yet clear, although some suggestions have been made by different research groups investigating this in primates or healthy adults. In monkeys, sustained activity in PFC and posterior association areas was found during maintenance of information in the retention interval of a visuo-spatial WM task (Miller, Erickson, \& Desimone, 1996). When intervening distractors were presented, the PFC sustained stimulus-selective activity whereas posterior association areas did not and this led to the conclusion that PFC plays an important role in protecting memory against distraction. Several fMRI studies investigating such WM-interference control interactions in healthy adults have found activation of similar PFC (DLPFC) -posterior brain networks during active WM maintenance in visuo-spatial (Sakai, et al., 2002) and non-spatial (Yoon, Curtis, \& D'Esposito, 2006) WM tasks. Both studies suggest that the protection of memory representations against distraction is established through higher-order interactions between prefrontal (DLPFC) and posterior association areas. The DLPFC is thought to be specifically involved in executive control in WM (manipulation, interference control), and is assumed to exert top-down control over posterior association areas that are involved in storage of memory representations. Yoon et al. (2006) showed disturbed functional connectivity between DLPFC and posterior association areas during distraction trials.

In light of these findings, the increased memory disruption during distraction in the 2-back task in childhood and adolescence in the present study might be the result of immaturity of the connections and communication between (dorsolateral) PFC and posterior association areas, instead of immaturity of PFC as such. Supportive of such a conclusion, using diffusion tensor imaging (DTI), Nagy, Westerberg and Klingberg (2004) reported that developmental increases in WM capacity were positively correlated with prefrontal-parietal connectivity. Further, findings from computational brain modeling by Edin, Macoveanu, Olesen, Tegner and Klingberg (2007) showed that, at the structural level, synaptic connections between cells in fronto-parietal networks explained the observed increase in brain activity associated with development of WM during adolescence. The abovemen- 
tioned studies, however, made no distinction between developmental differences in brain activation and connectivity patterns evoked by simple tasks involving only storage or maintenance, and more complex WM tasks involving higher-order (interference) control functions, thereby limiting the applicability of these results to the present findings.

Since the present study only used a verbal N-Back task, an important question is to what extent the results might generalize to spatial WM development. Smith and Jonides (1998) review fMRI and PET evidence for domain specific differences in functional networks activated during simple WM-tasks requiring low executive control. More specifically, they reported that the neural architecture of storage and rehearsal components, that are necessary for simple maintenance of information, differs between verbal and spatial tasks. However, during complex WM tasks that also demand high levels of attention or executive control, such as the 2-back task, activation of similar DLPFC-parietal networks for verbal and visuo-spatial tasks has been reported (Gevins et al., 1996; McEvoy, Smith, \& Gevins, 1998; Smith \& Jonides, 1998). In view of this literature, it seems reasonable to assume that the late development of executive-control-demanding WM-abilities will also be present for spatial tasks. Supportive evidence comes from reports of immature DLPFC functioning in childhood during performance of a spatial WM task (Scherf, et al., 2006) and highly comparable delays in development of verbal and spatial WM skills requiring high executive control (Conklin, et al., 2007). But to keep the type of executive control in spatial and verbal tasks exactly comparable future developmental studies should include verbal and spatial N-Back tasks and compare development across different load levels; also neurobiological measures should be included. If such a study would provide evidence for involvement of similar DLPFC-parietal networks in the development of executive working memory in both verbal and spatial tasks, this would be evidence in favor of the processspecific theory (Petrides, 1995). This theory states that dorsal regions of the PFC are particularly involved in complex WM skills such as manipulation, that require high levels of executive control.

Given the strong links between working memory (capacity) and cognitive functions as reading and mathematics (Pickering, 2006), it is important to discuss the practical implications of the current data. Our findings suggest that before their adolescent years, children experience disproportionate difficulties in tasks that call upon simultaneous maintenance and executive (interference) control processes. Considering that such concurrent cognitive operations are often required in everyday school-exercises, different or additional educational approaches might be useful for this population. For example, where possible, teachers might try to reduce secondary task demands during tasks requiring complex WM-skills. Furthermore, training in the use of WM-strategies might enhance the efficiency with which school-aged children can hold information online. This might lead to an increase of 
available resources that can be used for suppressing the influence of the simultaneously executed (interfering) operations, thereby protecting WM content against distraction.

In conclusion, to our knowledge, the present study is the first study comparing development of WM-capacity across a broad age range of 6-28 years, in simple and complex WM tasks ( $\mathrm{N}$-Back task varying in load) that do or do not require simultaneous interference control. It was demonstrated that the ability to maintain information in WM during the simultaneous suppression of irrelevant information (2back task) undergoes prolonged maturation into adolescence. In contrast, the ability to hold information online in tasks that demand no interference control (1-back task) reaches adult levels at age 10-12. Based on neuro-cognitive findings from the primate and adult literature (Miller, et al., 1996; Sakai, et al., 2002; Yoon, et al., 2006), this finding of prolonged development of WM maintenance during interference control was suggested to be linked to relative immaturity of functional connectivity between PFC (being involved in executive control in WM) and posterior association cortex where memory representations are stored.

\section{Limitations of the present study}

Several limitations of this study deserve further comment. Although the current behavioral findings have important implications for the developmental cognitive neuroscience field, direct brain-behavior links and developmental interactions herein cannot be derived from the present data. Future research should incorporate neuroimaging techniques in order to test more directly which network of brain regions supports development of WM performance during distraction or high executive control demanding situations. Another possible limitation concerns the cross-sectional nature of the study. This type of design does not allow us to make any causal interpretations of the observed results. The current findings await replication using a longitudinal design. 


\section{References}

Achenbach, T. M. (1991). Manual for the child behavior checklist/4-18 Burlington: University of Vermont, Departement of Psychiatry.

Baddeley, \& Hitch, G. J. (1974). Working memory. In G. A. Bower (Ed.), Recent advances in learning and motivation (Vol. 8, pp. 47-89). New York: Academic Press.

Baddeley, A. (1992). Working memory. Science, 255, 556-559.

Boice, R., \& Gardner, R. M. (1988). A Computer-Program to Generate Parametric and Nonparametric Signal-Detection Parameters. Bulletin of the Psychonomic Society, 26, 365-367.

Bunge, S. A., \& Wright, S. B. (2007). Neurodevelopmental changes in working memory and cognitive control. Current Opinion in Neurobiology, 17, 243-250.

Casey, B. J., Cohen, J. D., Jezzard, P., Turner, R., Noll, D. C., Trainor, R. J., et al. (1995). Activation of prefrontal cortex in children during a nonspatial working memory task with functional MRI. Neuroimage, 2, 221-229.

Casey, B. J., Giedd, J. N., \& Thomas, K. M. (2000). Structural and functional brain development and its relation to cognitive development. Biological psychology, 54, 241-257.

Chao, L. L., \& Knight, R. T. (1995). Human prefrontal lesions increase distractibility to irrelevant sensory inputs. Neuroreport, 6, 1605-1610.

Ciesielski, K. T., R.L., L., R.L., S., Grant, E. P., \& Ahlfors, S. P. (2006). Developmental neural networks in children performing a Categorical N-Back Task. Neuroimage, 33, 980-990.

Conklin, H. M., Luciana, M., Hooper, C. J., \& Yarger, R. S. (2007). Working memory performance in typically developing children and adolescents: behavioral evidence of protracted frontal lobe development. Developmental Neuropsychology, 31, 103-128.

Cowan, N. (1997). The development of memory in childhood. East Sussex, UK: Psychology Press.

Crone, E. A., Wendelken, C., Donohue, S., van Leijenhorst, L., \& Bunge, S. A. (2006). Neurocognitive development of the ability to manipulate information in working memory. Proceedings of the National Academy of Sciences of the United States of America, 103, 9315-9320.

D'Esposito, M., Detre, J. A., Alsop, D. C., Shin, R. K., Atlas, S., \& Grossman, M. (1995). The neural basis of the central executive system of working memory. Nature, 378, 279-281.

D'Esposito, M., Postle, B. R., Jonides, J., \& Smith, E. E. (1999). The neural substrate and temporal dynamics of interference effects in working memory as revealed by event-related functional MRI. Proceedings of the National Academy of Sciences of the United States of America, 96, 7514-7519.

de Fockert, J. W., Rees, G., Frith, C. D., \& Lavie, N. (2001). The role of working memory in visual selective attention. Science, 291, 1803-1806.

Diamond, A. (2002). Normal development of prefrontal cortex from birth to young adulthood: Cognitive functions, anatomy, and biochemistry. In D. T. Stuss, Knight, R.T. (Ed.), Principles of frontal lobe function. New York: Oxford University Press.

Edin, F., Macoveanu, J., Olesen, P., Tegner, J., \& Klingberg, T. (2007). Stronger synaptic connectivity as a mechanism behind development of working memory-related brain activity during childhood. Journal of Cognitive Neuroscience, 19, 750-760.

Eriksen, B. A., \& Eriksen, C. W. (1974). Effects of noise letters upon the identification of a target letter in a nonsearch task. Perception \& Psychophysics, 16, 143-149.

Gathercole, S. E. (1998). The development of memory. Journal of Child Psychology and Psychiatry and Allied Disciplines, 39, 3-27.

Gathercole, S. E. (1999). Cognitive approaches to the development of short-term memory. Trends in Cognitive Sciences, 3, 410-419.

Gathercole, S. E., Alloway, T. P., Kirkwood, H. J., Elliott, J. G., Holmes, J., \& Hilton, K. A. (2008). Attentional and executive function behaviours in children with poor working memory. Learning and Individual Differences, 18, 214-223. 
Gevins, A., Smith, M. E., Le, J., Leong, H., Bennett, J., Martin, N., et al. (1996). High resolution evoked potential imaging of the cortical dynamics of human working memory. Electroencephalography and Clinical Neurophysiology, 98, 327-348.

Giedd, J. N., Blumenthal, J., Jeffries, N. O., Castellanos, F. X., Liu, H., Zijdenbos, A., et al. (1999). Brain development during childhood and adolescence: a longitudinal MRI study. Nature Neuroscience, 2, 861-863.

Gogtay, N., Herman, D., Ordonez, A., Giedd, J., Hayashi, K., Greenstein, D., et al. (2004). Dynamic mapping of cortical brain development in pediatric bipolar illness. Neuropsychopharmacology, 29, S82-S82.

Groeger, J. A., Field, D., \& Hammond, S. M. (1999). Measuring memory span. International Journal of Psychology, 34, 359-363.

Hale, S. (1990). A Global Developmental Trend in Cognitive Processing Speed. Child Development, 61, 653-663.

Hollingshead, A. B. (1975). Four factor index of social status. New Haven, CT: Yale University of Department of Sociology.

Huizinga, M., Dolan, C. V., \& van der Molen, M. W. (2006). Age-related change in executive function: developmental trends and a latent variable analysis. Neuropsychologia, 44, 2017-2036.

Isaacs, E. B., Varga-Khadem, F. (1989). Differential course of development of spatial and verbal memory span: A normative study. British Journal of Developmental Psychology, 7, 377-380.

Jeyakumar, S. L. E., Warinner, E. M., Raval, V. V., \& Ahmad, S. A. (2004). Balancing the need for reliability and time efficiency: Short forms of the Wechsler Adult Intelligence Scale-III. Educational and Psychological Measurement, 64, 71-87.

Jongen, E. M., \& Jonkman, L. M. (2008). The developmental pattern of stimulus and response interference in a color-object Stroop task: an ERP study. BMC neuroscience, 9, 82.

Jonkman, L. M. (2006). The development of preparation, conflict monitoring and inhibition from early childhood to young adulthood: a Go/Nogo ERP study. Brain Research, 1097, 181-193.

Kail, R., \& Park, Y. S. (1992). Global Developmental-Change in Processing Time. Merrill-Palmer Quarterly-Journal of Developmental Psychology, 38, 525-541.

Kane, M. J., \& Engle, R. W. (2003). The contributions of goal neglect, response competition, and task set to Stroop interference. Journal of Experimental Psychology: General, 132, 47-70.

Kanemura, H., Aihara, M., Aoki, S., Araki, T., \& Nakazawa, S. (2003). Development of the prefrontal lobe in infants and children: a three-dimensional magnetic resonance volumetric study. [Comparative Study]. Brain \& development, 25, 195-199.

Kwon, H., Reiss, A. L., \& Menon, V. (2002). Neural basis of protracted developmental changes in visuospatial working memory. Proceedings of the National Academy of Sciences of the United States of America, 99, 13336-13341.

Lavie, N., Hirst, A., de Fockert, J. W., \& Viding, E. (2004). Load theory of selective attention and cognitive control. Journal of experimental psychology. General, 133, 339-354.

Luciana, M., \& Nelson, C. A. (1998). The functional emergence of prefrontally-guided working memory systems in four- to eight-year-old children. Neuropsychologia, 36, 273-293.

Luna, B., \& Sweeney, J. A. (2004). The emergence of collaborative brain function: FMRI studies of the development of response inhibition. Annals of the New York Academy of Sciences, 1021, 296-309.

Luna, B., Thulborn, K. R., Munoz, D. P., Merriam, E. P., Garver, K. E., Minshew, N. J., et al. (2001). Maturation of widely distributed brain function subserves cognitive development. Neuroimage, 13, 786-793.

McEvoy, L. K., Smith, M. E., \& Gevins, A. (1998). Dynamic cortical networks of verbal and spatial working memory: effects of memory load and task practice. Cerebral Cortex, 8, 563-574.

McNab, F., \& Klingberg, T. (2008). Prefrontal cortex and basal ganglia control access to working memory. Nature Neuroscience, 11, 103-107.

Miller, E. K., Erickson, C. A., \& Desimone, R. (1996). Neural mechanisms of visual working memory in prefrontal cortex of the macaque. The Journal of neuroscience : the official journal of the Society for Neuroscience, 16, 5154-5167. 
Nagy, Z., Westerberg, H., \& Klingberg, T. (2004). Maturation of white matter is associated with the development of cognitive functions during childhood. Journal of Cognitive Neuroscience, 16, 12271233.

Nichelli, F., Bulgheroni, S., \& Riva, D. (2001). Developmental patterns of verbal and visuospatial spans. Neurological sciences : official journal of the Italian Neurological Society and of the Italian Society of Clinical Neurophysiology, 22, 377-384.

Olesen, P. J., Macoveanu, J., Tegner, J., \& Klingberg, T. (2007). Brain activity related to working memory and distraction in children and adults. Cereb Cortex, 17, 1047-1054.

Pandya, D. N., Barnes, C.L. (1987). The frontal lobes revisited. In E. Perecman (Ed.), (pp. 41-72). New York: IRBN Press.

Petrides, M. (1995). Functional organization of the human frontal cortex for mnemonic processing. Evidence from neuroimaging studies. Annals of the New York Academy of Sciences, 769, 85-96.

Pickering, S. J. (2006). Working memory and education. San Diego, California: Academic Press.

Postle, B. R. (2005). Delay-period activity in the prefrontal cortex: one function is sensory gating. Journal of Cognitive Neuroscience, 17, 1679-1690.

Riggs, K. J., McTaggart, J., Simpson, A., \& Freeman, R. P. (2006). Changes in the capacity of visual working memory in 5- to 10-year-olds. Journal of Experimental Child Psychology, 95, 18-26.

Sakai, K., Rowe, J. B., \& Passingham, R. E. (2002). Active maintenance in prefrontal area 46 creates distractor-resistant memory. Nature Neuroscience, 5, 479-484.

Schacter, D. L., \& Slotnick, S. D. (2004). The cognitive neuroscience of memory distortion. Neuron, 44, 149-160.

Scherf, K. S., Sweeney, J. A., \& Luna, B. (2006). Brain basis of developmental change in visuospatial working memory. Journal of Cognitive Neuroscience, 18, 1045-1058.

Smith, E. E., \& Jonides, J. (1997). Working memory: a view from neuroimaging. Cognitive Psychology, 33, 5-42.

Smith, E. E., \& Jonides, J. (1998). Neuroimaging analyses of human working memory. Proceedings of the National Academy of Sciences of the United States of America, 95, 12061-12068.

Sowell, E. R., Thompson, P. M., Holmes, C. J., Batth, R., Jernigan, T. L., \& Toga, A. W. (1999). Localizing age-related changes in brain structure between childhood and adolescence using statistical parametric mapping. Neuroimage, 9, 587-597.

Spreen, O., Strauss, E., . (1998). A compendium of neuropsychological tests: Administration, norms, and commentary. New York: Oxford University Press.

Stins, J. F., Polderman, J. C., Boomsma, D. I., \& de Geus, E. J. (2005). Response interference and working memory in 12-year-old children. Child neuropsychology : a journal on normal and abnormal development in childhood and adolescence, 11, 191-201.

Towse, J. N., Hitch, G. J., \& Hutton, U. (2002). On the nature of the relationship between processing activity and item retention in children. Journal of Experimental Child Psychology, 82, 156-184.

Ullman, R. K., Sleator, E. K., \& Spraque, R. L. (1991). Manual for the ADD-H comprehensive Teacher's Rating Scale (ACTeRS). St. Louis, MO: MetriTech.

Van Leijenhorst, L., Crone, E. A., \& Van der Molen, M. W. (2007). Developmental trends for object and spatial working memory: a psychophysiological analysis. Child Development, 78, 987-1000.

Vuontela, V., Steenari, M. R., Carlson, S., Koivisto, J., Fjallberg, M., \& Aronen, E. T. (2003). Audiospatial and visuospatial working memory in 6-13 year old school children. Learning \& memory, 10, 7481.

Wechsler, D. (1991). Wechsler Intelligence Scale for Children-Third Edition. New York: Psychological Corporation.

Yoon, J. H., Curtis, C. E., \& D'Esposito, M. (2006). Differential effects of distraction during working memory on delay-period activity in the prefrontal cortex and the visual association cortex. Neuroimage, 29, 1117-1126. 


\section{Chapter 3}

\section{Children's use of semantic organizational strategies is mediated by working memory capacity}

Corresponding publication:

Schleepen, T.M.J., \& Jonkman, L.M. (2012). Children's use of semantic organizational strategies is mediated by working memory capacity. Cognitive Development, 27, 255-269. 


\begin{abstract}
In adults, the ability to apply semantic grouping strategies has been found to depend on working memory. To investigate this relation in children, two sort-recall tasks (one without and one with a grouping instruction) were administered to 612-year-olds. The role of working memory was examined by means of mediation analyses and by assessing whether children who successfully used the semantic grouping strategy had higher working memory capacity than did children who did not show such strategy use. Only children aged 8-12 were able to successfully use semantic grouping strategies (and 8-9-year-olds only after instruction), while strategy use was absent in 6-7-year-olds. Both types of analysis involving working memory suggested that, also in children, working memory (and not short-term memory) mediates the development of successful use of the semantic grouping strategy during both encoding and retrieval.
\end{abstract}




\section{Introduction}

Storage of information in long-term memory (LTM) can be improved by the use of strategies that organize information in working memory (WM) prior to encoding. Storage of information in meaningful groups facilitates later retrieval (Dehn, 2008; Shiffrin \& Atkinson, 1969; Tulving, 1962). Such groupings may be based on perceptual similarities between items, such as a similar color or shape, or on semantic relatedness, such as belonging to the semantic category "animals" (Lange, Guttentag, \& Nida, 1990; Melkman, Tversky, \& Baratz, 1981). Effective employment of grouping strategies aids children both in daily activities and in academic contexts. For example, children's organization of basic math facts in LTM helps them to recall those facts later to solve larger, more complex mathematical problems (Geary \& Brown, 1991). Learning-disabled children have been reported to make less use of semantic organizational strategies in a free-recall task and show poorer recall (Bauer, 1977; Torgesen, 1977).

It is thus important to identify the factors involved in children's ability to apply intentional grouping strategies. The present study examines the role of age and conditions and, in particular, the role of working memory capacity (WMC). By about age 7 , children begin to use elementary memory strategies, such as rehearsal (Ornstein, Baker-Ward, \& Naus, 1988; Ornstein \& Naus, 1978). Preschoolers spontaneously group material based on overlap in perceptual features but not on semantic relationships (Melkman, et al., 1981). When items have strong, well-learned semantic associations (e.g., cow-milk), grouping strategies are reported to emerge relatively early, at around age 9 . However, when items have low within-category associations (e.g., bird-dolphin), spontaneous application of the semantic grouping strategy is only reported beginning at age 13 (Bjorklund \& de Marchena, 1984; Bjorklund \& Jacobs, 1985).

Such developmental differences can be explained by differing demands on mental resources. When associations are well-learned, they are activated more or less automatically when seeing or hearing stimuli, requiring no active-grouping strategy (Schneider \& Pressley, 1997). When associations between stimuli are less well-learned, one must actively search for and encode such relationships in WM (Baddeley, 2000; Daneman \& Carpenter, 1980). Because WM has limited capacity and is subject to development, WM may affect children's ability to actively apply semantic memory-grouping strategies. Late development of this ability (Bjorklund \& de Marchena, 1984; Bjorklund \& Jacobs, 1985) parallels the recently reported prolonged maturational course of WM into adolescence (Conklin, Luciana, Hooper, \& Yarger, 2007; Diamond, Kirkham, \& Amso, 2002; Schleepen \& Jonkman, 2010). The neurobiological source of this late development has been identified as the protracted development of a network of prefrontal and parietal brain regions that undergo considerable structural and functional changes throughout this period 
(Bunge \& Wright, 2007; Luna, Padmanabhan, \& O'Hearn, 2010). Furthermore, WM plays an important role in learning complex cognitive activities involving language, mathematics, and reasoning (Andersson \& Lyxell, 2007; Barrouillet \& Lepine, 2005; Noel, 2009; Pickering, 2006), perhaps involving strategy use.

A link between WM and the productive application of grouping strategies has been shown in adults. Individuals with high WM span are more likely than those with a low span to employ a semantic clustering strategy when retrieving information from LTM in a verbal fluency task (Rosen \& Engle, 1997), and those with more effective semantic memory strategies show superior WM (McNamara \& Scott, 2001).

To our knowledge, only two studies have explored relations between WM and semantic grouping in children. Developmental studies have often made use of "sort-recall" tasks (Schneider \& Pressley, 1997) that typically consists of two phases. In the first phase, children are presented with randomly ordered pictures of objects that belong to different semantic or perceptual categories. They are instructed to study these pictures for later recall and told they may move the pictures if they think it will help them remember. After a short "buffer-clearing" interval, in a second phase children are asked to verbally report as many pictures as they can. The level of grouping on perceptual or semantic relatedness a child engages in is evaluated by computing so-called clustering scores (Roenker, Thompson, \& Brown, 1971).

Using a sort-recall task, Schneider, Kron, Hunnerkopf and Krajewski (2004) studied differences in WM among school-aged children who were consistently strategic or utilization deficient (UD) in the use of the semantic-grouping strategy. Children were assessed at two time points and classified as UD if their sorting behavior increased significantly across time without corresponding increases in recall. Consistent strategy users were those children who applied the sorting strategy at both points and demonstrated consistently high recall. UD children had lower WM scores (measured by the digit span backward task) than consistent strategy users. In a follow-up study including nine longitudinal measurement points, KronSperl, Schneider and Hasselhorn (2008) reported that individual differences in short-term memory (STM) span, but not WMC, predicted recall performance in 810 -year-olds who spontaneously used the semantic grouping strategy. There are, however, several factors that might explain the absence of a WM-semantic memory grouping relation in this study. The to-be-grouped items were highly associated, so that perhaps no deliberate strategy application (requiring WM) was needed, as noted earlier.

In conclusion, there is evidence that WM affects adults' ability to deliberately apply semantic organization or grouping strategies. There is, however, a lack of information about such relations in children that may be potentially important as argued earlier. The present study investigates such relations by presenting a sort- 
recall task to children 6-12 years of age. The to-be-learned pictures belonged to different color or semantic categories. Only pictures with low within-category associations (e.g., dog-monkey instead of dog-cat) were used so as to examine the development of deliberate, effort-demanding, grouping-strategy application that has been shown to depend on WM in adults.

Our main goal was to study whether children who successfully implemented the semantic- grouping strategy at the encoding and retrieval phase (strategic children) had higher WMC than children who did not show such strategy use (nonstrategic children). In some studies, children were classified as "strategic" based on above-chance clustering scores during sorting only. Although sorting during study has regularly been used as a measure of conscious strategy use (Schneider \& Pressley,1997), and earlier studies reported positive correlations between sorting scores and recall, high sorting scores alone do not assure that the sorted items were also actively encoded and retrieved in semantic groups in LTM. Sortingrelated increases in recall may be caused by factors other than active encoding in semantic groups, for example by having more intensively processed the items during sorting. In the present study, we therefore classified children as strategic/nonstrategic based on their sorting behavior and also according to their use of clustering during retrieval. High semantic clustering scores during retrieval of material that is not highly associated is thought to indicate deliberate, effortful use of the grouping strategy (Bjorklund, Coyle, \& Gaultney, 1992; Bjorklund \& de Marchena, 1984; Bjorklund \& Harnishfeger, 1987).

Another of the study's aims was to analyze developmental differences. At what age are normally developing children able to spontaneously use perceptual or semantic-grouping strategies and does this use lead to higher recall? Before children become able to use a memory strategy spontaneously and successfully, they may experience (at least) two types of deficiencies. During the first, mediationdeficiency phase (Reese, 1962), children do not spontaneously use a memory strategy and cannot be prompted to do so. During the second, productiondeficiency phase (Flavell, 1970), children do not engage in spontaneous strategy use but are able to use it successfully after prompting. To examine the occurrence of mediation versus production deficiencies, all children performed the sort-recall task both without and with a nondirective grouping prompt. Earlier studies have shown that children aged 4-7 need elaborate training or explicit prompting about the to-be-grouped categories to be able to use and benefit from grouping strategies (Carr \& Schneider, 1991; Lange, et al., 1990; Lange \& Pierce, 1992; Moeley, Olson, Halwes, \& Flavell, 1969). The present study adds to this literature by examining whether and at what age children might benefit from a single nondirective grouping prompt. It might be that from a certain age, children have sufficient skills and capacity to apply the strategy but do not spontaneously use it. These children 
might benefit from a single nondirective grouping prompt without requiring extensive training.

\section{Method}

\section{Participants}

A total of 83 participants from a primary school were divided into three age groups, 6-7, 8-9 and 10-12 years; these groupings were based on the knowledge discussed earlier regarding age trends in strategy use.

Demographic characteristics (age, group size, gender, IQ and attention scores and socioeconomic status) are presented in Table 1. IQ scores were derived from a short form of the Wechsler Intelligence Scale for Children (WISC-III, Dutch version), including the vocabulary and block design subtests, that correlates .9 with the full test (Jeyakumar, Warinner, Raval, \& Ahmad, 2004; Spreen, 1998). Children with IQ scores below 80 were excluded. Children with diagnosed childhood disorders like ADHD, ODD, CD, autism, or learning disorders, and children taking medication, were excluded from participation. Because attention problems are highly related to WM deficits, effort was made to exclude undiagnosed attention deficits by allowing parents to fill out the Child Behavior Checklist (CBCL; Achenbach, 1991). Children scoring above the clinical threshold on the attention subscale were excluded. A total of 0 children were excluded from participation based on one or more of these criteria.

Table 1. Demographic characteristics per age group.

\begin{tabular}{lccc}
\hline & \multicolumn{3}{c}{ Age Group } \\
\cline { 2 - 4 } Variable & $6-7$ & $8-9$ & $10-12$ \\
\hline$N$ & 28 & 30 & 25 \\
Gendera $^{\text {(\% female) }}$ & 50 & 57 & 64 \\
Age & $6.6(.50)$ & $8.6(.50)$ & $11.0(.82)$ \\
Attention score & $54.2(4.2)$ & $54.6(4.5)$ & $54.5(4.2)$ \\
Estimated IQ $^{\text {c }}$ & $109.8(13.5)$ & $107.8(13.4)$ & $107.6(21.4)$ \\
SES $^{\text {d }}$ & $5.5(2.0)$ & $6.2(1.4)$ & $5.3(1.8)$ \\
\hline
\end{tabular}

Note. Standard deviations are depicted between brackets. aThere were no significant differences in gender between age groups $\left(\chi^{2}(2)=1.05, p>.1\right.$. ${ }^{b}$ None of the participants scored above the clinical threshold on the attention subscale of the CBCL. CBCL attention scores did not significantly differ between age groups $(F(2,82)=.072, p>.1)$. cIQ did not significantly differ between age groups $(F(2,82)=.25$, $p>$.1). dSocioeconomic status (SES) was determined by Hollingshead (1975) occupational scale for the parent holding the higher status job ( 1 or $2=$ unskilled or unemployed positions, 3 or $4=$ skilled or semiskilled labourers, 5 or $6=$ managerial professions, 8 or $9=$ major professions). Parental occupation data was not available for one child in each age group. SES was not significantly different between age groups $(F(2,79)=1.8, p>1)$. 


\section{Procedure}

Children were tested in a quiet room at their school in a single experimental session lasting approximately $2 \mathrm{~h}$. Tasks were administered in a constant order. In addition to the tasks reported on here, children performed a computerized WM task that was administered in two separate parts. Each session began with the first 4 blocks of a computer-presented memory task, followed by the first sort-recall task. No grouping instructions were provided, and the child's performance was intended to reflect spontaneous use of grouping strategies. After children completed the remaining 3 blocks of the memory task, a second sort-recall task was presented in which children were prompted to apply a grouping strategy. At the end of the session, children completed the short-form WISC-III and digit-span forward and backward tests.

Before the start of the first sort-recall task, the cards were placed on a 3-rowby-4-column array and covered with a cloth. The layout of the cards was similar for all participants, with the restriction that no two items from the same semantic or color category lay adjacently. The experimenter uncovered the cards and children were instructed to label the pictures to verify that they were familiar with each object. If a child did not know an object, the experimenter provided the correct name but took care not to provide any reference to the corresponding semantic category. Because all of the pictures were chosen based on familiarity rates for 56-year-olds (Cycowicz, Friedman, Rothstein, \& Snodgrass, 1997), it was rare that a child did not know the correct name of a picture. Children were then instructed to remember as many pictures as possible. They were told that the pictures would be removed after a while, after which they would be asked to name as many pictures as possible. They were also told they were allowed to move the pictures in any way that might help their recall.

Following a 75-s study period, the experimenter made a photograph of the arrangement of the pictures. The experimenter then removed the pictures from the child's view and children were instructed to count up to 50 for a period of $30 \mathrm{~s}$. This served as a buffer-clearing task to control for any recency or primacy effects. Children were then asked to recall as many items as possible. Recall was voice recorded. After the first silent period of $10 \mathrm{~s}$ in which the child did not report any additional pictures, the experimenter asked whether there were any more pictures that the child could remember. After another continued silence of $15 \mathrm{~s}$, the task was ended. 
Table 2. Stimulus items per category in the two sort-recall tasks.

\begin{tabular}{lll}
\hline Semantic category & Standard task & Instruction task \\
\hline Fruit & Peer (Pear) & Ananas (Pineaple) \\
& Aardbei (Strawberry) & Kers (Cherry) \\
& Citroen (Lemon) & Meloen (Melon) \\
& Druiven (Grapes) & Appel (Apple) \\
Animals & Dolfijn (Dolphin) & Kat (Cat) \\
& Hond (Dog) & Olifant (Elephant) \\
& Vogel (Bird) & Koe (Cow) \\
& Aap (Monkey) & Zwaan (Swan) \\
Clothes & Jas (Coat) & Hoed (Hat) \\
& Rok (Skirt) & Broek (Trousers) \\
& Das (Tie) & Want (Glove) \\
& Sok (Sock) & Jurk (Dress) \\
\hline
\end{tabular}

The second sort-recall task was identical with the exception that, before the start of this task, children were told that it might be easier to remember the pictures if they placed the pictures in groups that belong together. This instruction is considered nondirective because no hints were given as to the perceptual or semantic categories into which pictures could be grouped. At the end of this task, children were checked for color-blindness by asking them to label the four colors that were used.

\section{Tasks}

\section{Sort-recall task}

The material for the sort-recall task comprised two stimulus sets of 12 black-andwhite line drawings of objects printed on $6 \mathrm{~cm} \times 5 \mathrm{~cm}$ cards. The borders of the cards $(0.2 \mathrm{~cm})$ were printed in one of four colors (green, blue, brown or gray). The objects in each set could be grouped according to semantic category (four objects from three distinct categories; see Table 2) and/or by color. For each stimulus set, different pictures of objects (from the same semantic categories) were used, and the two stimulus sets were counterbalanced across the two sort-recall tasks and across age groups.

The choice of line drawings in the two stimulus sets was based on several considerations. First, semantic categories of stimuli were selected that were common to children within this age range. This selection was undertaken based on familiarity rates for 5- and 6-year-olds (Cycowicz, et al., 1997). This procedure 
yielded highly and equally familiar objects from the three semantic categories. Second, only object stimuli were included that consisted of 3-7-letter words. This restriction ensured that differences in recall performance could not be attributed to the fact that short-item words are better recalled than long-item words (Neath, Bireta, \& Surprenant, 2003). Finally, the least-associated items within each category were selected to constitute the two stimulus sets. This selection was accomplished by means of a pilot study with adults in which all possible combinations of pairings of two pictures from the same semantic category were presented to a group of 13 adults. These adults rated the level of association between items on a 10 -point scale ( 1 = low association and $10=$ high association). Mean association scores for all pairs per category fell between 2.3 and 2.7 (SD between 1.5 and 1.9). Adults were instructed that the extent of association could be based on different features and that the ratings should be based on their first impressions. The rationale for including adults in this pilot study was that if adults, with their elaborate knowledge bases, were to rate item pairs as having a low semantic association, one can assume this would be the case for children.

\section{Short-term memory and working memory tasks}

The digit-span forward and digit-span backward tests, adapted from the Wechsler Intelligence Scale for Children (WISC-III, Dutch version), were used as measures of STM and WMC (Gathercole, Pickering, Ambridge, \& Wearing, 2004; St ClairThompson, 2010).

\section{Scoring strategy use}

The procedures for scoring strategy use were similar to those used in most prior research. Adjusted ratio of clustering (ARC) scores (Roenker, et al., 1971) were used to assess the amount of color or semantic sorting during study and clustering at retrieval. The ARC score does not vary systematically with the number of recalled items (Murphy, 1979), making it an appropriate measure of strategy use when different levels of recall are expected for different groups, as in the present case. In the present study two types of clustering (ARC) scores are calculated. First, the sorting ARC score that reflects strategy use during the study phase of the task and reflects the extent to which pictures from the same color or semantic category are sorted in groups above a certain chance level. Second, we calculated an ARC retrieval clustering score by counting the words belonging to the same color or semantic category that were recalled in successive order above a certain chance level during the retrieval phase of the task. ARC scores vary between -1 and 1 , with a score of 1 reflecting perfect sorting or retrieval clustering, a score of zero indicating sorting or retrieval clustering at chance level, and a score below zero 
representing sorting or retrieval clustering below chance level. In calculating the ARC sorting score, a repetition was counted if two pictures of the same semantic category or color were laid above, below or next to one another. Intrusions and perseverations were not included in calculating the ARC retrieval scores. Following Coyle and Bjorklund (1997), sorting during study or clustering at retrieval was considered to be meaningful (i.e., children were considered strategic) when clustering scores were equal to or greater than .5. An ARC score of .5 reflects a value of slightly more than one standard deviation greater than sorting or clustering expected by chance.

\section{Results}

Due to non-normal distributions, all data were log-transformed prior to statistical analysis. Because no meaningful patterns appeared for color sorting or retrieval (ARC scores <.5), only analyses for semantic sorting and retrieval are reported.

\section{Developmental differences in the sort-recall task}

Table 3 presents mean ARC scores for semantic sorting during study and clustering during retrieval, mean recall, and percentage who sorted or clustered above chance levels (ARC scores $\geq .5$ ), as a function of age and instruction condition. Mixed design repeated measures ANOVA's were performed with age (6-7, 8-9, and 10-12) as between-subjects factor and instruction (no instruction, grouping instruction) as within-subjects factor to study development of strategy use and effects of instruction.

\section{Sorting during study}

Analysis of semantic sorting scores during study yielded main effects of instruction (no instruction vs. grouping instruction) and age group (6-7, 8-9, and 10-12), $F(2,80)=45.8, p<.00001, \eta 2 p=.36$, and $F(2,80)=9.5, p=.001, \eta 2 p=.19$, respectively. Semantic sorting was higher in the instructed task than in the non-instructed standard task $(p<.00001)$. Post hoc analyses showed that semantic sorting scores were higher among 10-12-year olds than among either 8-9-year-olds or 6-7-yearolds $(p<.05$ and $p<.001$, respectively), who did not significantly differ from each other $(p=.45)$. Mean group ARC scores (Table 3 ) indicate that, before instruction, semantic sorting approached meaningful levels only among 10-12-year-olds. After instruction, both 8-9-year-olds and 10-12-year-olds had meaningful ARC sorting scores, whereas 6-7-year-olds did not sort meaningfully before or after instruction. 


\section{Clustering at retrieval}

For clustering at retrieval, main effects of instruction and age were found, $F(2,80)=15.5, p<.001, \eta 2 p=.16$, and $F(2,80)=12.9, p<.0001, \eta 2 p=.25$, respectively. Semantic clustering at retrieval was higher in the instructed task than in the noninstructed task $(p<.001)$. Scores were higher among 10-12-year-olds than 8-9year-olds or 6-7-year-olds ( $p<.01$ and $p<.00001$, respectively), and the latter two groups did not differ significantly from one another $(p=.51)$. Before instruction, semantic ARC retrieval scores were meaningful (ARC $\geq .5$ ) only in 10-12-year-olds (Table 3). After instruction, both 8-9-year-olds and 10-12-year-olds had meaningful ARC retrieval scores, while scores were below chance levels among 6-7-yearolds both before and after instruction.

\section{Recall}

A significant age $\times$ instruction interaction was found for recall scores, $F(2,80)=3.6$, $p<.05, \eta 2 \mathrm{p}=.08$. A main age effect appeared for the non-instructed task, $F(2,80)=11.8, p<.0001, \eta 2 p=.23$. The 10-12-year-olds showed higher recall than either 6-7- or 8-9-year-olds ( $p<.0001$ and $p<.01$, respectively). Recall performance did not significantly differ between 6-7- and 8-9-year-olds $(p=.59)$. For the instructed task, a main effect of age was found, $F(2,80)=17.6, p<.00001, \eta 2 p=.31$. The $8-9$-year-olds had recall scores similar to 10-12 year-olds $(\mathrm{p}=.13)$. Both groups showed higher recall than 6-7 year-olds ( $\mathrm{p}<.001$ and $\mathrm{p}<.00001$, respectively).

Table 3. Mean semantic sorting and retrieval clustering scores and percentages of children showing meaningful strategy use (ARC scores $>.5$ ) by age and instruction condition.

\begin{tabular}{|c|c|c|c|c|c|c|c|c|c|}
\hline \multirow[b]{3}{*}{ Short-term memory } & \multicolumn{9}{|c|}{ Age Group } \\
\hline & \multicolumn{3}{|c|}{ 6-7 } & \multicolumn{3}{|c|}{$8-9$} & \multicolumn{3}{|c|}{$10-12$} \\
\hline & 6.5 & $(1.4)$ & & 6.8 & $(1.4)$ & & 7.9 & $(1.3)$ & \\
\hline Working memory & 3.4 & $(1.0)$ & & 4.7 & $(1.4)$ & & 5.3 & $(2.0)$ & \\
\hline \multicolumn{10}{|l|}{ Standard task } \\
\hline Sorting & -.13 & $(.55)$ & $18 \%$ & -.08 & $(.58)$ & $23 \%$ & .41 & $(.69)$ & $60 \%$ \\
\hline Clustering & .01 & $(.53)$ & $18 \%$ & -.01 & $(.51)$ & $17 \%$ & .52 & $(.61)$ & $68 \%$ \\
\hline Recall & 7.6 & $(2.3)$ & & 8.2 & $(2.0)$ & & 10.5 & $(1.8)$ & \\
\hline \multicolumn{10}{|l|}{ Instruction task } \\
\hline semantic sorting & .27 & $(.61)$ & $46 \%$ & .59 & $(.60)$ & $70 \%$ & .86 & $(.36)$ & $92 \%$ \\
\hline semantic clustering & .21 & $(.56)$ & $39 \%$ & .51 & $(.51)$ & $57 \%$ & .76 & $(.32)$ & $80 \%$ \\
\hline Recall & 6.6 & $(2.4)$ & & 8.8 & $(2.3)$ & & 10.3 & $(1.5)$ & \\
\hline
\end{tabular}

Note. Short-term memory is assessed by the WISC forward digit span task and working memory by the WISC backward digit span task 
Relations among age, strategy use and recall

Correlations are presented in Table 4. Significant positive correlations between ARC sorting and recall scores and between ARC retrieval and recall scores were found in 10-12-year-olds in both standard and instructed task (except that the correlation between ARC sorting and recall was not significant in the instructed task). In 8-9-year-olds, these correlations only reached significance in the instructed task, while 6-7-year-olds showed no significant correlations in either task. ARC sorting scores were positively correlated with ARC retrieval scores in all age groups in both standard and instructed tasks, except that among 6-7-yearolds, this correlation was non-significant in the standard task. In sum, neither of the instruction conditions led to above-chance semantic sorting or retrieval clustering scores among the youngest children, indicating that they were mediation deficient. The 8-9-year-olds did not spontaneously sort or cluster according to semantic category but had above chance sorting and retrieval clustering scores after having received a nondirective grouping prompt. This prompt was also related to higher recall, showing they were production deficient. The 10-12-year-olds had above-chance sorting and retrieval-clustering scores in both instruction conditions that were consistently related to higher recall, demonstrating that they were consistently strategic.

\section{The role of STM/WM in successfully using semantic grouping strategies}

To investigate the role of STM and/or WMC in the successful use of the semantic grouping strategy, we performed two types of analyses. First, we performed mediation analyses to examine whether STM/WMC is a significant mediating factor in the relationship between semantic strategy use and recall. Second, we performed a between-group analysis to test whether children who successfully used the semantic grouping strategy had higher STM and/or WMC than children who did not use this strategy.

\section{Mediation analyses}

We followed recommendations from Baron and Kenny (1986), who stated that a full mediation analysis involves studying the following three relations: (1) between the independent variable and the mediator, (2) between the independent variable and the dependent variable, and (3) between the mediator and the dependent variable after controlling for the independent variable. If these are significant, the Sobel test (Sobel, 1982) can be performed to determine whether the effect of the independent variable on the dependent variable is significantly less when the effect of the mediator is taken into account. The mediation analysis was performed 
over the full sample $(\mathrm{n}=83)$, based on recommendations by Mackinnon, Warsi and Dwyer (1995) that a minimal sample size of 50 is required to obtain sufficient power to detect significant mediation effects. To include both spontaneously strategic and production deficient children, the mediation analysis was performed on data from the instructed condition.

Table 4. Pearson's correlations between sorting during study, clustering at retrieval, recall and shortterm memory (STM) and Working memory (WM) by task and age group.

\begin{tabular}{|c|c|c|c|}
\hline & \multicolumn{3}{|c|}{ Age group } \\
\hline & $6-7$ & $8-9$ & $10-12$ \\
\hline STM-WMC & -.02 & .15 & .03 \\
\hline \multicolumn{4}{|l|}{ standard task } \\
\hline sorting-clustering & .30 & $.46^{* *}$ & $.45^{*}$ \\
\hline sorting-recall & .16 & .24 & $.59 * *$ \\
\hline clustering-recall & -.15 & .10 & $.61^{* *}$ \\
\hline STM-sorting & -.01 & .25 & .22 \\
\hline STM-clustering & -.09 & $.46^{*}$ & .01 \\
\hline STM-recall & -.04 & .01 & .32 \\
\hline WM-sorting & .15 & .19 & .09 \\
\hline WM-clustering & -.05 & .08 & .21 \\
\hline WM-recall & .08 & .23 & $.38^{* * *}$ \\
\hline \multicolumn{4}{|l|}{ instruction task } \\
\hline sorting-clustering & $.49^{* *}$ & $.52^{* *}$ & $.54^{* *}$ \\
\hline sorting-recall & .25 & $.42^{*}$ & .07 \\
\hline clustering-recall & .13 & $.40^{*}$ & $.45^{*}$ \\
\hline STM-sorting & -.10 & .23 & .24 \\
\hline STM-clustering & .02 & -.04 & .28 \\
\hline STM-recall & -.09 & -.07 & .24 \\
\hline WM-sorting & $.40^{*}$ & -.24 & -.02 \\
\hline WM-clustering & .20 & $\therefore .00$ & .20 \\
\hline WM-recall & .24 & .07 & $.42^{*}$ \\
\hline
\end{tabular}

$* p<.05$

$* * p<.01$

${ }^{* * *} p=.064$ (trend-significant)

For STM, correlations for both sorting and retrieval showed that not all three criteria were met to perform the Sobel test (for sorting, the first correlation was not significant, while for retrieval clustering, the third correlation was not significant). Thus, STM did not mediate the relationship between sorting and recall or between clustering at retrieval and recall. Concerning WMC, for the sorting strategy a bor- 
derline significant correlation was found between ARC scores and WMC $(r=.20$, $p=.074)$ and a significant correlation between ARC scores and recall $(r=.42$, $p<.0001)$ and between WMC and recall after controlling for sorting $(r=.38, p<.001)$. Despite the borderline-significant first correlation, the Sobel test was performed, revealing that WMC did not mediate the relation between sorting and recall, $z=1.58, p>.1$. For the clustering retrieval strategy, significant correlations were found between (1) ARC retrieval scores and WMC ( $r=.31, p<.01)$, (2) ARC retrieval scores and recall $(r=.51, p<.00001)$, and (3) WMC and recall after controlling for clustering at retrieval $(r=.33, p<.01)$. As all three criteria for mediation were met, the Sobel test was performed and showed a significant mediation effect, $z=2.05$, $p<.05$, indicating that WMC was a significant mediator in the relation between clustering at retrieval and recall.

\section{Differences in STM/WMC between strategy and non-strategy users}

These analyses involved several steps. First, children were divided into two groups of strategy or non-strategy users independent of age. A child was assigned to the strategic group when ARC scores were $\geq .5$ (see Table 3). Strategy groups were formed separately for sorting and clustering retrieval strategies. The assignment to strategy groups was based on sorting and retrieval clustering scores in the instructed condition so as to include production-deficient children. Second, by applying Mann-Whitney tests, we examined whether strategy users and non-strategy users differed in their recall performance and STM/WMC. Third, the relationship between recall and STM or WMC was examined by computing correlations between recall and digit-span forward (STM) and backward (WM scores separately for strategy and non-strategy users. For all analyses, two-tailed significance levels of $p<.05$ were adopted.

For the sorting strategy, there were 57 strategic and 26 non-strategic children. For the clustering retrieval strategy, there were 48 strategic and 35 non-strategic children. Mann-Whitney tests showed significantly higher recall among children who applied the sorting strategy, $\mathrm{U}=1060, \mathrm{p}<.01$ and children who applied the clustering retrieval strategy, $U=1276, p<.0001$, than among those who did not. Furthermore, whereas forward digit-span scores were borderline higher among strategic sorters compared to nonstrategic sorters, $U=932, p=.056$, forward digitspan scores did not differ between children who did or did not apply the clustering retrieval strategy, $U=981, p>.1$ (see Fig. 1). In contrast, backward digit-span scores did not significantly differ between children who did or did not use the sorting strategy, $U=855, p>.1$, while children who applied the clustering retrieval strategy had significantly higher backward digit-span scores than children who did not use this strategy, $U=1088, p<.05$ (Fig. 1). 
Again, there were no significant correlations between STM and recall in the strategic or nonstrategic groups ( $p s>.1$ ). As expected, WMC did correlate significantly with recall among children who successfully applied the clustering retrieval strategy $(r=.40, p<.01)$ but not in non-strategic clusterers ( $p>.1)$. The WM-recall relationship was also significant among the group of strategic sorters $(r=.45, p<.001)$ while not among non-sorters ( $p>.1$ ). This finding is likely due to the large overlap (42 participants) between strategic sorting and clustering retrieval groups. A hierarchical regression analysis in the group of strategic sorters, with WMC entered in the first block and ARC sorting and ARC retrieval scores in the second block, showed that WMC explained 20\% $(\mathrm{p}<.001)$ and retrieval clustering an additional $21.6 \%(\mathrm{p}<.0001)$ of the variance in the recall scores of the strategic sorters, whereas sorting did not explain any additional variance. Figure 2 shows the scatter-plots that depict the correlation between WMC and recall in all strategic and nonstrategic groups.

Finally, to also investigate the role of STM and WMC in the prediction of recall performance above strategy use in the entire sample, we performed a regression analysis entering semantic sorting and clustering retrieval scores in the first step and STM and WM scores in the second step. This analysis demonstrates that WMC (but not STM) additionally explains $9.2 \%(p<.01)$ of the variance in recall scores above semantic sorting and clustering retrieval scores.

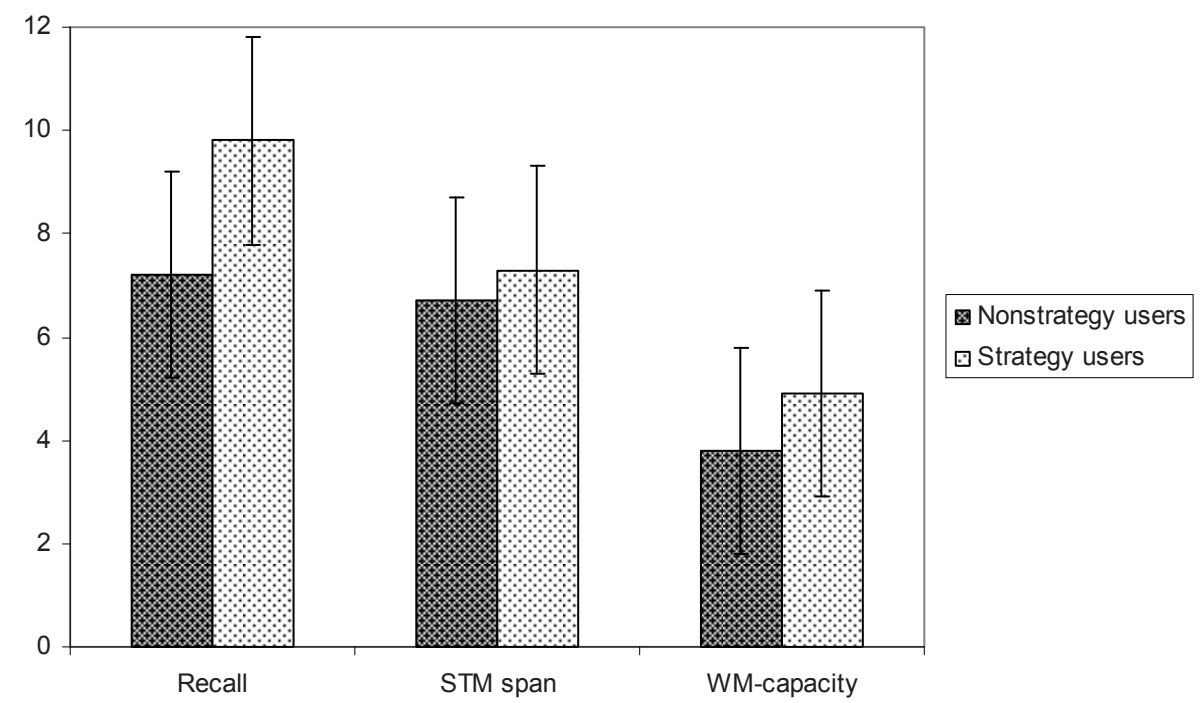

Figure 1. Mean recall, STM and WM scores among strategy users and nonstrategy users. 


\section{Discussion}

The primary aim of the present study was to investigate whether working memory is an important determinant of the efficient use of semantic grouping strategies in children. A second aim was to investigate the mean age at which children begin to spontaneously use color or semantic grouping strategies that improve memory, along with the age at which a single nondirective grouping instruction leads to the successful application of the strategy in non-spontaneous users. This was investigated by having 6-12-year-old children perform a sort-recall task with pictures that belonged to different color or semantic categories. The tasks were performed twice, once without and once with a nondirective prompt in which children were told that grouping pictures that belong together might improve recall (the categories were not mentioned).

Results showed that only 10-12-year-olds were able to spontaneously and successfully use the semantic grouping strategy. Semantic grouping strategy use was absent in 6-7-year-olds, while children aged 8-9 only (successfully) used this strategy after prompting. The role of STM and/or WMC (as inferred from forward and backward digit-span tests, respectively) was investigated by performing mediation analyses and testing whether strategy users had higher recall and higher STM/WM than non-strategy users. Both types of analysis suggested that WMC (and not STM) mediates the development of successful use of the semantic grouping strategy.

\section{Developmental differences in the use of semantic grouping strategies}

Color-grouping scores during sorting and retrieval were below chance levels in all age groups and will not be further discussed. Developmental differences in the deliberate application of the semantic organizational strategy and the role of WM were investigated by only including items that had low associations within their category (Bjorklund \& de Marchena, 1984; Bjorklund \& Jacobs, 1985). During the study phase, the categorization of less-associated items into semantic categories demanded that children consciously and deliberately search for categories and to subsequently actively encode the items in these categories. These categories could then be used as retrieval cues to facilitate later recall. By comparing sorting, clustering retrieval and recall scores across age groups in the sort-recall task without instruction, information was obtained regarding the age at which children showed spontaneous strategy use. Below-chance ARC sorting and clustering retrieval scores were found in 6-7-year-olds and 8-9-year-olds, signifying that they did not spontaneously sort or retrieve the pictures in semantic categories. In contrast, among 10-12-year-olds, sorting and retrieval-clustering scores were above chance level, and sorting, clustering and recall scores were significantly higher than 
among 6-9-year-olds. Moreover, sorting, clustering retrieval and recall scores significantly correlated with each other only among 10-12-year-olds, demonstrating that strategy use was successful. After receiving a non-directive grouping prompt, the 6-7-year-olds still showed no meaningful sorting during study or retrieval, and their recall performance was significantly lower than that of older children. However, among (spontaneously nonstrategic) 8-9-year-olds, the nondirective grouping prompt caused them to become as strategic as the (spontaneously strategic) 10-12-year-olds. Additionally, positive correlations between sorting during study, clustering at retrieval and recall appeared in both 8-9- and 1012-year-olds.

Integrating the results from both instruction conditions, it can be concluded that 6-7-year-olds were mediation deficient: they did not spontaneously use memory strategies and could not be prompted to do so (Bjorklund, Miller, Coyle, \& Slawinski, 1997). In contrast, 8-9-year-olds were production deficient: they only used the strategy after prompting but with recall benefits (for review, see Schneider \& Pressley, 1997). Finally, 10-12-year-olds were consistently strategic, also without prompting.

The finding of the absence of spontaneous use of the semantic grouping strategy before age 9 is consistent with earlier findings (Bjorklund \& de Marchena, 1984; Bjorklund \& Jacobs, 1985). The start of spontaneous semantic grouping from age 10 is in line with a study by (Moeley, et al., 1969) but in contrast with findings by Bjorklund and colleagues (Bjorklund \& de Marchena, 1984; D.F. Bjorklund \& Jacobs, 1985), who reported effective use of semantic organization strategies with loosely associated materials only beginning at age 13. Schneider, Borkowski, Kurtz and Kerwin (1986) reported that 9-year-old German children, but not 9-year-old American children, were able to spontaneously use the semantic organization strategy. These different findings might indicate that European children show earlier emergence of the semantic grouping strategy, possibly due to differences in teaching practices or metacognitive awareness (Kurtz, Schneider, Carr, Borkowski, \& Rellinger, 1990).

The present results add to the memory-strategy training literature by demonstrating that, whereas 8-9-year-olds do not use semantic organization strategies spontaneously, they do not need extensive training in strategy use to do so effectively; a single prompt to group pictures that belong together appears to be sufficient at this age. Children younger than 8 years, however, may need more extensive training to achieve semantic grouping skills. This finding is consistent with the results of earlier studies that have demonstrated that with elaborate training, including demonstration and practice in using semantic features to encode and retrieve information, as well as receiving feedback, 4-7-year-olds were able to apply semantic organization strategies and demonstrate enhanced recall (Carr \& Schneider, 1991; Lange, et al., 1990; Lange \& Pierce, 1992; Stevens, 2002). The 
reason that a single non-directive grouping prompt only triggered strategy use from age 8 onward might be that around this age, important development in awareness about representations and their logical relatedness is taking place (Demetriou \& Kazi, 2006; Demetriou, Spanoudis, \& Mouyi, 2011).

\section{Roles of short-term and working memory in children's effective semantic strategy use}

Two types of analyses were performed to answer the main research question of the degree to which STM and/or WMC contribute to the ability to successfully apply semantic grouping strategies. Both indicated that, while the successful application of the sorting strategy placed no significant demands on WMC (or STM) in 612-year-olds, successful application of the clustering-retrieval strategy was dependent on WMC and not on STM. This conclusion was based on the following findings: (1) STM neither mediated the relation between sorting and recall nor between clustering at retrieval and recall, (2) WM only mediated the relation between clustering at retrieval and recall, and not between sorting during study and recall, (3) WMC (not STM) was significantly higher in children who successfully applied the semantic grouping strategy during retrieval than in children who did not do so, while there were no significant WMC or STM differences between sorters and non-sorters and (4) WMC correlated with recall in both the strategic sorting and clustering retrieval groups (but not in the two non-strategic groups). However, there was a large overlap among strategic sorters and clusterers $73 \%$ of the strategic sorters also used the semantic clustering strategy during retrieval), and a regression analysis indicated that only clustering-retrieval scores (not sorting scores) explained additional variance in recall above WM-span scores in sorters. The finding that the application of the sorting strategy consumes little of children's WM resources is in line with a study by Kron-Sperl, et al. (2008), who reported that STM (not WMC) significantly predicted recall in children who consistently applied the sorting strategy. In our study, STM differences between sorters and non-sorters were only borderline significant. The low dependency of sorting on WMC might mean that with loosely associated materials, sorting does not automatically lead to active encoding (and subsequent retrieval) of items in semantic clusters. Thus far, WM has been found to play an important role in semantic memory strategy use in adults (Gaultney, Kipp, \& Kirk, 2005; McNamara \& Scott, 2001; Rosen \& Engle, 1997) and in cumulative rehearsal strategies in children (Lehmann \& Hasselhorn, 2007). The present findings add to this literature by demonstrating the importance of WMC in the efficient use of semantic organization strategies during encoding and retrieval among children.

The present data do not answer the question of how WM mediates recall in children who make efficient use of semantic organization strategies. One can, however, speculate in this regard on the basis of prior studies in adults. During the 
encoding of categorized word lists, the central executive component of WM (Baddeley \& Hitch, 1974) is thought to play a role in mediating organizational strategies (Gershberg \& Shimamura, 1995) and in maintaining constant conscious awareness of the to-be-encoded targets (Moscovitch, 1992, 1994). During the retrieval of categorized lists, the central executive is only needed during controlled search for earlier stored category names, which would then automatically trigger the category items (Cinan, 2003; Rosen \& Engle, 1997). Dual-task studies in adults that manipulated available executive resources showed that when the number of to-beretrieved category names is low, minimal demands are placed on the central executive during retrieval (Cinan, 2003; Johnston, Rollie, \& Griffith, 1972). During encoding, however, the effects of performing a secondary task that consumed central executive resources was much stronger and always compromised recall, independent of the number of categories in the to-be-learned list (Cinan, 2003). In light of these results, we hypothesize that WM influences children's recall by mediating the influences on strategy use and attention regulation during the encoding phase of the task. Future work, manipulating categories and the availability of resources could further examine this hypothesis.

Whereas the present results show that children need sufficient WMC to be able to apply semantic grouping strategies with success (i.e., with recall benefits), other developmental studies suggest that improvements in WM performance or cognitive control are in themselves caused by developmental changes in cognitive flexibility or increased levels of consciousness. For instance, Camos and Barrouillet (2011) concluded that improvements in WM performance between 7 and 9 years of age were due to increases in cognitive (attentional) flexibility enabling older children to regularly switch attention between maintenance and processing, preventing the decay of information with time. The same cognitive flexibility that is needed in WM tasks might be necessary to switch between the processing of the items in categories and the maintenance (rehearsal) of already-stored items in the sort-recall task. Zelazo (2004) introduced his levels of consciousness (LOC) model that describes age-related changes in the degree of self-reflection that children can display, which is thought to have consequences for the potential for recall and the complexity of children's explicit knowledge structures. When children do not enter a certain (higher) level of consciousness on their own, they might be triggered to do so if provided with labels or prompts. Applying this model to our findings, the grouping prompt might have triggered 8-9-year-olds to reflect on their representations at a higher consciousness level, making them aware of the semantic associations, which they subsequently encoded. The system might have been insufficiently mature among 6-7-year-olds to achieve a higher level of consciousness based on our non-specific prompt.

Effort-demanding memory strategies such as semantic organization are fundamental for academic learning and everyday functioning. Those undertaking in- 
terventions to enhance children's use of such strategies might determine whether children have weaknesses in WM. If so, training can be aimed at increasing WMC (Dahlin, Backman, Neely, \& Nyberg, 2009; Klingberg, 2010; Morrison \& Chein, 2011), thereby promoting acquisition of efficient organizational memory skills. Also, remedial teachers should screen for WM impairments at an early age so that training can be introduced if indicated. Such efforts are important because children who are able to use more efficient strategies may have an advantage in the learning and application of cognitive skills (Bauer, 1977). 


\section{References}

Achenbach, T. M. (1991). Manual for the child behavior checklist/4-18 Burlington: University of Vermont, Departement of Psychiatry.

Andersson, U., \& Lyxell, B. (2007). Working memory deficit in children with mathematical difficulties: a general or specific deficit? Journal of Experimental Child Psychology, 96, 197-228.

Baddeley. (2000). The episodic buffer: a new component of working memory? Trends in Cognitive Sciences, 4, 417-423.

Baddeley, \& Hitch, G. J. (1974). Working memory. In G. A. Bower (Ed.), Recent advances in learning and motivation (Vol. 8, pp. 47-89). New York: Academic Press.

Baron, R. M., \& Kenny, D. A. (1986). The moderator-mediator variable distinction in social psychological research: conceptual, strategic, and statistical considerations. Journal of Personality and Social Psychology, 51, 1173-1182.

Barrouillet, P., \& Lepine, R. (2005). Working memory and children's use of retrieval to solve addition problems. Journal of Experimental Child Psychology, 91, 183-204.

Bauer, R. H. (1977). Memory processes in children with learning disabilities: evidence for deficient rehearsal. Journal of Experimental Child Psychology, 24, 415-430.

Bjorklund, D. F., Coyle, T. R., \& Gaultney, J. F. (1992). Developmental Differences in the Acquisition and Maintenance of an Organizational Strategy - Evidence for the Utilization Deficiency Hypothesis. Journal of Experimental Child Psychology, 54, 434-448.

Bjorklund, D. F., \& de Marchena, M. R. (1984). Developmental shifts in the basis of organization in memory: The role of associative versus categorical relatedness in children's free recall. Child Development, 55, 952-962.

Bjorklund, D. F., \& Harnishfeger, K. K. (1987). Developmental differences in the mental effort requirements for the use of an organizational strategy in free recall. Journal of Experimental Child Psychology, 44, 109-125.

Bjorklund, D. F., \& Jacobs, J. W. (1985). Associative and categorical processes in children's memory: The role of automaticity in the development of organization in free recall. Journal of Experimental Child Psychology, 39, 599-617.

Bjorklund, D. F., Miller, P. H., Coyle, T. R., \& Slawinski, J. L. (1997). Instructing children to use memory strategies: Evidence of utilization deficiencies in memory training studies. Developmental Review, 17, 411-441.

Bunge, S. A., \& Wright, S. B. (2007). Neurodevelopmental changes in working memory and cognitive control. Current Opinion in Neurobiology, 17, 243-250.

Camos, V., \& Barrouillet, P. (2011). Developmental change in working memory strategies: from passive maintenance to active refreshing. Developmental Psychology, 47, 898-904.

Carr, M., \& Schneider, W. (1991). Long-Term Maintenance of Organizational Strategies in KindergartenChildren. Contemporary Educational Psychology, 16, 61-72.

Cinan, S. (2003). Executive processing in free recall of categorized lists. Learning and Motivation, 34, 240-261.

Conklin, H. M., Luciana, M., Hooper, C. J., \& Yarger, R. S. (2007). Working memory performance in typically developing children and adolescents: behavioral evidence of protracted frontal lobe development. Developmental Neuropsychology, 31, 103-128.

Coyle, T. R., \& Bjorklund, D. F. (1997). Age differences in, and consequences of, multiple- and variablestrategy use on a multitrial sort-recall task. Developmental Psychology, 33, 372-380.

Cycowicz, Y. M., Friedman, D., Rothstein, M., \& Snodgrass, J. G. (1997). Picture naming by young children: Norms for name agreement, familiarity, and visual complexity. Journal of Experimental Child Psychology, 65, 171-237. 
Dahlin, E., Backman, L., Neely, A. S., \& Nyberg, L. (2009). Training of the executive component of working memory: subcortical areas mediate transfer effects. Restorative Neurology and Neuroscience, 27, 405-419.

Daneman, M., \& Carpenter, P. A. (1980). Individual differences in working memory and reading. Journal of verbal learning and verbal behavior, 19, 450-466.

Dehn, M. J. (2008). Working memory and academic learning: Assesment and intervention. Hoboken, New Jersey: Wiley \& Sons, Inc.

Demetriou, A., \& Kazi, S. (2006). Self-awareness in g (with processing efficiency and reasoning). Intelligence, 34, 297-317.

Demetriou, A., Spanoudis, G., \& Mouyi, A. (2011). Educating the developing mind: towards an overarching paradigm. Educational Psychology Review, 1-63.

Diamond, A., Kirkham, N., \& Amso, D. (2002). Conditions under which young children can hold two rules in mind and inhibit a prepotent response. Developmental Psychology, 38, 352-362.

Flavell, J. H. (1970). Developmental studies of mediated memory. In H. W. Reese \& L. P. Lipsitt (Eds.), Advances in child development and behavior (Vol. 5, pp. 181-211). New York: Academic.

Gathercole, S. E., Pickering, S. J., Ambridge, B., \& Wearing, H. (2004). The structure of working memory from 4 to 15 years of age. Developmental Psychology, 40, 177-190.

Gaultney, J. F., Kipp, K., \& Kirk, G. (2005). Utilization deficiency and working memory capacity in adult memory performance: not just for children anymore. Cognitive Development, 20, 205-213.

Geary, C. G., \& Brown, S. C. (1991). Cognition addition: Strategy choice and speed of processing differences in gifted, normal, and mathematically disabled children. Developmental Psychology, 27, 398-406.

Gershberg, F. B., \& Shimamura, A. P. (1995). Impaired use of organizational strategies in free recall following frontal lobe damage. Neuropsychologia, 33, 1305-1333.

Hollingshead, A. B. (1975). Four factor index of social status. New Haven, CT: Yale University of Department of Sociology.

Jeyakumar, S. L. E., Warinner, E. M., Raval, V. V., \& Ahmad, S. A. (2004). Balancing the need for reliability and time efficiency: Short forms of the Wechsler Adult Intelligence Scale-III. Educational and Psychological Measurement, 64, 71-87.

Johnston, W. A., Rollie, R. W., \& Griffith, D. (1972). Information-processing analysis of verbal learning. . Journal of Experimental Psychology, 96, 307-314.

Klingberg, T. (2010). Training and plasticity of working memory. Trends in Cognitive Science, 14, 317 324.

Kron-Sperl, V., Schneider, W., \& Hasselhorn, M. (2008). The development and effectiveness of memory strategies in kindergarten and elementary school: Findings from the Wurzburg and Gottingen longitudinal memory studies. Cognitive Development, 23, 79-104.

Kurtz, B. E., Schneider, W., Carr, M., Borkowski, J. G., \& Rellinger, E. (1990). Strategy Instruction and Attributional Beliefs in West-Germany and the United-States - Do Teachers Foster Metacognitive Development. Contemporary Educational Psychology, 15, 268-283.

Lange, G., Guttentag, R. E., \& Nida, R. E. (1990). Relationships between study organization, retrieval organization, and general and strategy-specific memory knowledge in young children. Journal of Experimental Child Psychology, 49, 126-146.

Lange, G., \& Pierce, S. H. (1992). Memory-Strategy Learning and Maintenance in Preschool-Children. Developmental Psychology, 28, 453-462.

Lehmann, M., \& Hasselhorn, M. (2007). Variable memory strategy use in children's adaptive intratask learning behavior: Developmental changes and working memory influences in free recall. Child Development, 78, 1068-1082.

Luna, B., Padmanabhan, A., \& O'Hearn, K. (2010). What has fMRI told us about the Development of Cognitive Control through Adolescence? Brain and Cognition, 72, 101-113.

Mackinnon, D. P., Warsi, G., \& Dwyer, J. H. (1995). A Simulation Study of Mediated Effect Measures. Multivariate Behavorial Research, 30, 41. 
McNamara, D. S., \& Scott, J. L. (2001). Working memory capacity and strategy use. Memory \& Cognition, 29, 10-17.

Melkman, R., Tversky, B., \& Baratz, D. (1981). Developmental trends in the use of perceptual and conceptual attributes in grouping, clustering, and retrieval. Journal of Experimental Child Psychology, 31, 470-486.

Moeley, B. E., Olson, F. A., Halwes, T. G., \& Flavell, J. H. (1969). Production deficieny in young children's clustered recall. Developmental Psychology, 1, 26-34.

Morrison, A. B., \& Chein, J. M. (2011). Does working memory training work? The promise and challenges of enhancing cognition by training working memory. Psychonomic Buletin \& Review, 18, 46-60.

Moscovitch, M. (1992). Memory and Working-with-Memory - a Component Process Model Based on Modules and Central Systems. Journal of Cognitive Neuroscience, 4, 257-267.

Moscovitch, M. (1994). Cognitive resources and dual-task interference effects at retrieval in normal people: The role of the frontal lobes and medial temporal cortex. Neuropsychology, 8, 524-534.

Murphy, M. (1979). Measure of category clustering in free recall. In R. Puff (Ed.), Memory organization and structure (pp. 51-81). New York: Academic Press.

Neath, I., Bireta, T. J., \& Surprenant, A. M. (2003). The time-based word length effect and stimulus set specificity. Psychonomic Bulletin \& Review, 10, 430-434.

Noel, M. P. (2009). Counting on working memory when learning to count and to add: a preschool study. Developmental Psychology, 45, 1630-1643.

Ornstein, P. A., Baker-Ward, L., \& Naus, M. J. (1988). The development of mnenomic skill. In O. A. Barbarin \& B. H. Wasik (Eds.), Handbook of child development and early education: Research to practice (pp. 103-122). New York: Guilford.

Ornstein, P. A., \& Naus, M. J. (1978). Rehearsal processes in children's memory. In P. A. Ornstein (Ed.), Memory development in children (pp. 69-99). Hillsdale, New York: Erlbaum.

Pickering, S. J. (2006). Working memory and education. San Diego, California: Academic Press.

Reese, H. W. (1962). Verbal mediation as a function of age level. Psychological Bulletin, 59, 502-509.

Roenker, D. L., Thompson, C. P., \& Brown, S. C. (1971). Comparison of measures for the estimation of clustering in free recall. Psychological Bulletin, 76, 45-48.

Rosen, V. M., \& Engle, R. W. (1997). The role of working memory capacity in retrieval. Journal of Experimental Psychology-General, 126, 211-227.

Schleepen, T. M. J., \& Jonkman, L. M. (2010). The development of non-spatial working memory capacity during childhood and adolescence and the role of interference control: An N-Back task study. Developmental Neuropsychology, 35, 37-56.

Schneider, W., Borkowski, J. G., Kurtz, B. E., \& Kerwin, K. (1986). Metamemory and motivation: A comparison of strategy use and performance in German and American children. Journal of CrossCultural Psychology, 17, 315-336.

Schneider, W., Kron, V., Hunnerkopf, M., \& Krajewski, K. (2004). The development of young children's memory strategies: First findings from the Wurzburg Longitudinal Memory Study. Journal of Experimental Child Psychology, 88, 193-209.

Schneider, W., \& Pressley, M. (1997). Memory development between two and twenty (2nd ed.). Mahwah, New Jersey: Lawrence Erlbaum Associates.

Shiffrin, R., \& Atkinson, R. C. (1969). Storage and retrieval processes in long-term memory. Psychological Review, 76, 179-193.

Sobel, M. E. (1982). Asymptotic intervals for indirect effects in structural equations models. In S. Leinhart (Ed.), Sociological methodology (pp. 290-312). San Francisco: Jossey-Bass.

Spreen, O., Strauss, E., . (1998). A compendium of neuropsychological tests: Administration, norms, and commentary. New York: Oxford University Press.

St Clair-Thompson, H. L. (2010). Backwards digit recall: A measure of short-term memory or working memory? European Journal of Cognitive Psychology, 22, 286-297.

Stevens, J. P. (2002). Applied multivariate statistics for the social sciences (fourth ed.). London: Lawrence Erlbaum Associates, Publishers. 
Torgesen, J. K. (1977). Memorization processes in reading-disabled children. Journal of Educational Psychology, 69, 571-578.

Tulving, E. (1962). Subjective organization in free recall of "unrelated" words. Psychological Review, 69, 344-354.

Zelazo, P. D. (2004). The development of conscious control in childhood. Trends in Cognitive Science, 8, 12-17. 


\section{Chapter 4}

\section{A longitudinal study of semantic grouping strategy use in 6-12 year-old children: investigating developmental phases, strategy transfer and the role of working memory}

Corresponding manuscript

Schleepen, T.M.J., \& Jonkman, L.M. (submitted). A longitudinal study of semantic grouping strategy use in 6-12 year-old children: investigating developmental phases, strategy transfer and the role of working memory 


\begin{abstract}
This longitudinal study on the development of the semantic grouping strategy had three goals. First, we examined if 6-7-year-old children first pass through the mediation deficiency (MD) phase before they enter the production deficiency (PD) phase at the age of 8-9, and if 8-9-year-old children first pass through the PD phase before they are able to consistently use the semantic grouping strategy at the age of 10-12. To this end, children aged 6-7 and 8-9-years performed two sort-recall tasks (one without and one with a grouping instruction) at two time points separated 1,5 years from each other. Second, we investigated whether PD children that only showed successful strategy use after prompting and children who used the strategy spontaneously differed in strategy transfer to a new task at time point 2 . Third, we investigated whether short term- or working memory capacity at time point 1 predicted recall in children who did or did not use the semantic grouping strategy 1,5 years later. The current longitudinal study confirmed findings from prior cross-sectional studies that 6-7-year-olds first are MD before they enter the PD phase when they are 8-9-years-old, and that 8-9-year-olds first pass through the PD phase before they are consistently strategic at the age of 10-12. Further, we found that PD children were equally able as consistently strategy children to successfully use the semantic grouping strategy on the transfer task. Finally, a WMCsemantic strategy use relationship could not be confirmed longitudinally.
\end{abstract}




\section{Introduction}

Grouping or organizing to-be-remembered information in memory (e.g. on semantic category or color) can be helpful to remember a large amount of material so that it can be easily recalled. Use of such strategies often leads to improved memory performance, and children who make less use of them may be at risk for developing learning difficulties (Bauer, 1977). Since memory grouping strategies are so important, especially for children who still need to acquire a lot of academic skills, it is of great importance to identify at what age children are able to use them, and if and how such strategy use can be improved in children who do not spontaneously use them. In this longitudinal study we will therefore investigate 1) what phases in spontaneous strategy use children pass between the age of 6 to 12 years, 2) how a single general grouping prompt influences this developmental pattern and if it can lead to consistent strategy use (measured by transfer to a new task) and 3) what role STM/WM capacity plays in longitudinal strategy development. These issues have not, or only partly, been investigated using a longitudinal design.

\section{Development of semantic grouping strategies}

Most of the conducted studies on the development of memory grouping strategies focused on semantic grouping which requires the grouping of to-be-learned material on semantic category in memory (e.g. the semantic category fruits). The age at which children start to use this strategy depends on the extent to which the to-belearned material is associated with each other. When items have strong, welllearned semantic associations (e.g. cow-milk), children of approximately 9 years are able to group this kind of information. Grouping of less well-learned material with low within category associations (e.g. bird-dolphin) requires considerably more effort and has been reported to develop only from the age of 13 years (Bjorklund \& de Marchena, 1984; Bjorklund \& Jacobs, 1985). Prior developmental cross-sectional studies showed that children pass through several stages before they are able to successfully apply the semantic grouping strategy, that is, when its use leads to enhanced recall. One phase that has received much attention in the developmental literature is the utilization deficiency (UD) phase in which a child is able to produce a strategy but does not benefit from it, i.e. does not show improved recall performance (Bjorklund, Miller, Coyle, \& Slawinski, 1997; Schwenck, Bjorklund, \& Schneider, 2009). The existence of UDs when children acquire memory grouping strategies has mainly been reported in cross-sectional studies (Bjorklund \& Coyle, 1995; Schwenck, et al., 2009). In the few longitudinal studies that have been done by Schneider and colleagues, UDs were found in only a small minority of children (Kron-Sperl, Schneider, \& Hasselhorn, 2008; Schneider, Kron- 
Sperl, \& Hunnerkopf, 2009; Schneider, Kron, Hunnerkopf, \& Krajewski, 2004) or were not found at all (Schlagmuller \& Schneider, 2002).

Besides the UD phase, cross-sectional studies have reported two other developmental phases that children pass through before they are able to spontaneously and successfully use a memory grouping strategy (Flavell, 1970; Reese, 1962). In the first, mediation deficiency (MD) phase that is observed in children of 6-to 7years of age, children are not able to spontaneously use semantic grouping strategies (Bjorklund \& de Marchena, 1984) and cannot be prompted or trained to do so (Schleepen \& Jonkman, 2012). The MD phase is followed by the production deficiency (PD) phase that is typically seen in 8- to 9-year-old children; these children do also not engage in spontaneous strategy use but are able to do so successfully after prompting (Flavell, 1970). Until now there are, to the best of our knowledge, no longitudinal studies that investigated if 6- to 7- year-old children first pass through the MD phase and then through the PD phase when they are 8- to 9-years old, and if 8- to 9- year-old-children first pass through the PD phase before they are able to spontaneously use semantic grouping strategies at age 10 to 12 . One of the aims of the present study was to investigate this.

\section{Strategy transfer}

Another aim of the present study was to examine strategy transfer in PD children that just acquired the semantic grouping strategy after receiving a single grouping prompting to determine the consistency of this developmental change. As mentioned above, PD children are (usually 8- to 9-year-old; Schleepen \& Jonkman, 2012) children who do not use a memory strategy spontaneously but can be successfully trained or prompted to do so. An interesting question is whether PD children are able to generalize their recently acquired semantic grouping skills to a new task when not prompted. Although relatively little is known about this issue, there are two studies that provide some information about this. A study by Ringel and Springer (1980) examined strategy transfer of the sorting strategy in 7, 9 and 11 year-old children and showed that 7 and 9-year-old children only showed strategy transfer to a sort-recall task containing new pictures after having received elaborate instructions, practice and feedback on strategy use. The 11-year-old children only needed instructions and practice in strategy use (and not feedback) to show strategy transfer. In another study by Schwenck, et al. (2009), 4- to 8-yearold children were trained in the use of a sorting strategy (i.e. group items during study), a clustering retrieval strategy (i.e. group items during recall) or received no training. Transfer was assessed two weeks later on a sort-recall task containing new pictures and new semantic categories. These authors used multivariate cluster analysis to identify subgroups of children that follow a similar developmental pattern regarding memory strategy acquisition, independent of age and experi- 
mental condition. They found that children who were classified as PD were not able to generalize the semantic grouping strategy to the transfer task.

Thus, the studies of Ringel and Springer (1980) and Schwenck et al. (2009) provide inconsistent results about strategy transfer in PD children, but this might be due to the inclusion of different age groups in both studies. Results from a prior cross-sectional study (Schleepen \& Jonkman, 2012) suggested that the critical age for being able to successfully apply a semantic grouping strategy after prompting is about 8-9 years, an age group not included in the study by Schwenck, et al., 2009. In this study it was also shown that 8-9 year-old children do not always need extensive training to develop strategy use, since they demonstrated successful application of the semantic grouping strategy after only receiving a single general grouping prompt, in which no grouping categories were mentioned, also no feedback was provided. It is however as yet unclear how consistent this strategy acquisition is, e.g. if there is transfer to a new task. This will be investigated in the present longitudinal study by comparing strategy transfer between PD children who just started to use the strategy after a single non-directive grouping prompt and consistently strategic children who did not need prompting. Just as in the prior studies near-transfer was studied, that is, transfer of the semantic grouping strategy to a task that is structurally similar to the task performed before instruction or training (i.e. sort-recall task, but new pictures and new semantic categories).

\section{Role of WMC in semantic grouping strategy use}

Besides a child's developmental phase, also working memory capacity (WMC), i.e. our ability to simultaneously maintain and manipulate information in mind (Baddeley, 2000) has been shown to be important for successfully using the semantic grouping strategy (Schleepen \& Jonkman, 2012). This relation has not only been demonstrated in children, but also in adults (McNamara \& Scott, 2001; Rosen \& Engle, 1997). Given the strong links between WMC and semantic strategy use and to extend findings from existing cross-sectional studies, the third aim of the current study was to investigate if WMC predicts semantic strategy use in children longitudinally. This is important since establishing which cognitive factors predict successful strategy use is crucial for early screening and intervention.

As far as we know, there are three longitudinal studies that investigated the role of STM/WMC (inferred from digit span forward and backward scores) in semantic strategy use in children, all from the group of Schneider and colleagues (Kron-Sperl, et al., 2008; Schneider, et al., 2009; Schneider, et al., 2004). In their first study it was shown that consistent strategy users had higher WMC than utilization deficient children, but these groups only contained 9 vs. 7 subjects, respectively (Schneider, et al., 2004). Further, whereas the 2008 study showed that differences in STM contributed significantly to recall performance (Kron-Sperl, et al., 
2008), the follow-up study in 2009 did not replicate this relationship (Schneider, et al., 2009). Also, these studies assessed the relation between STM/WMC and strategy use per grade or measurement point, and thus did not examine if STM or WMC predicts semantic strategy use longitudinally. This is important as mentioned earlier and therefore we also addressed this in the current study. A former study investigated the involvement of STM and WMC in the development of semantic grouping strategy use, in 6-12 year-old children using a cross-sectional design (Schleepen \& Jonkman, 2012). It was found that WMC was a predictor for success of using the retrieval cluster strategy (WMC significantly mediated retrieval clustering-recall relations), but did not play a role in successful use of the sorting strategy. In the current study we aimed to replicate this finding, but now using a longitudinal design.

\section{Methods}

\section{Participants}

A total of 51 children, recruited from one primary school, were tested at both time points. At time point 1, 83 children took part in the study (the cross-sectional developmental data from these 83 children at time point 1 was published in Schleepen and Jonkman (2012), but 32 children did not participate at time point 2 that took place 1.5 years later. Of these 32 children, 18 children moved to high school and 14 parents did not return the informed consent for participation of their child at time point 2 . The 51 children of which longitudinal data were obtained were divided in two age groups of children that were 6-to 7 and 8-9-yearsold at time-point 1 . The rationale for this division was based on the earlier crosssectional study showing that the 6-7 year-old children were MD and the 8-9 yearold children were PD (Schleepen \& Jonkman, 2012). The allocation of children in two age groups of 6-7- and 8-9-years-old children allow us to answer our research question if 6-7-years-old children first pass through the MD phase before entering the PD phase when they are 8- to 9-years old, and if 8- to 9-years-old children first pass through the PD phase before they are able to spontaneously and successfully use the semantic grouping strategy at age 10- to 12 .

Demographic characteristics (age, group size, gender, IQ, CBCL attention scores and socioeconomic status) for both age groups are shown in Table 1. IQ scores were derived from a short form of the Wechsler Intelligence Scale for Children (WISC-III, Dutch version) administered at time point 1 , including vocabulary and block design subtests, that correlates .9 with the full test (Jeyakumar, Warinner, Raval, \& Ahmad, 2004; Spreen, 1998). Exclusion criteria were: 1) presence of psychiatric or neurological disorders (e.g.: ADHD, ODD, CD, autism, learning disor- 
ders), 2) medication use, 3) an IQ-score below 80 (i.e. IQ-scores < 80 generally indicate a degree of mental retardation; Wechsler, 1991) and 4) a score above the clinical threshold on the attention subscale of the Child Behavior Checklist (CBCL; Achenbach (1991) filled out by the parents of the children at time point 1 . Because attention problems are highly related to WM deficits, effort was made to exclude undiagnosed attention deficits by allowing parents to fill out the Child Behavior Checklist. Scores on the CBCL-attention subscale were taken as an index of attention deficits; conform guidelines; T-scores between 67-70 were considered as borderline-clinical and T-scores $>70$ as clinical. None of the children met one or more of the above exclusion criteria. The study was approved based on procedures of a local ethical committee of Maastricht University, Faculty of Psychology and Neuroscience. Parental informed consent was obtained from all children.

Table 1. Demographic characteristics per age group. The ages of the children groups represent the ages at the first measurement point.

\begin{tabular}{|c|c|c|c|c|}
\hline \multirow[b]{3}{*}{ Variabele } & \multicolumn{4}{|c|}{ Age group } \\
\hline & $6-7$ & & 8-9 & \\
\hline & & & & \\
\hline$N$ & 26 & & 25 & \\
\hline Gender ( $\%$ female) & 34.6 & & 52 & \\
\hline Age & 6.7 & $(.71)$ & 8.6 & $(.75)$ \\
\hline Attention score ${ }^{b}$ & 54.8 & $(4.5)$ & 54.6 & $(4.3)$ \\
\hline Estimated IQc & 108.2 & $(13.4)$ & 106.3 & $(12.7)$ \\
\hline SES ${ }^{d}$ & 5.9 & (1.9) & 6.0 & $(1.5)$ \\
\hline
\end{tabular}

Note. Standard deviations are depicted between brackets. aGender did not significantly differ between age groups $\left(\chi^{2}(1)=1.6, p>1\right.$. ${ }^{b}$ None of the children scored above the clinical threshold on the attention subscale of the CBCL. For one child in the 6-7 group there were no CBCL scores available. CBCL attention scores did not significantly differ between age groups, $t(48)=.09, p>$.1. cIQ was not significantly

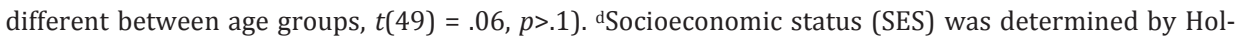
lingshead (1975) occupational scale for the parent holding the higher status job (1 or 2 = unskilled or unemployed positions, 3 or 4 = skilled or semiskilled labourers, 5 or 6 = managerial professions, 8 or 9 = major professions). Parental occupation data was not available for one child in the 6-7 group. There was no significant difference in SES between age groups, $t(48)=-.33, p>.1$

\section{Procedure}

The two test sessions (separated by 1.5 years) took place in a quiet room at the children's school, lasting about 2 hours each. In both sessions, tasks were administered in a constant order. In addition to the tasks reported on here, children performed a computerized working memory task that was administered in two separate parts during the sessions. Each session began with the first 4 blocks of the 
computerized memory task, followed by the first sort-recall task. In this task (i.e. the standard task), no grouping instruction was provided and the child's performance was intended to reflect spontaneous use of grouping strategies. After children completed the remaining 3 blocks of the computerized task, a second sortrecall task (i.e. the instruction task) was presented in which children were prompted to apply a grouping strategy. At the end of each session, children completed the short form WISC-III and digit span forward and backward tests. Children were rewarded with a small toy present. See Table 2 for a time schedule showing which tasks were administered at time point 1 and time point 2 .

The procedure for the two sort-recall tasks at both time points was as follows: before the start of the first sort-recall task, the cards were placed on a 3 row by 4 column array on the table and covered with a cloth. The layout of the cards was similar across participants, with the restriction that no two items from the same semantic or color category laid adjacently. After the experimenter uncovered the cards, children were instructed to label the pictures to verify that they were familiar with each object. If a child did not know an object, the experimenter provided the correct name but took care not to provide any reference to the corresponding semantic category. Because all of the pictures were chosen based on familiarity rates for 5-6 year-old-children (Cycowicz, Friedman, Rothstein, \& Snodgrass, 1997), it was very rare that a child did not know the correct name of a picture. Children were then instructed to look at and study the pictures in order to remember as many pictures as possible and were told that the pictures would be removed after a while, after which they would be asked to verbally report as many pictures as they still remembered. They were also told that they were allowed to move the pictures in any way that might help them remembering.

Following a 75-s study period, the experimenter made a photograph of the arrangement of the pictures. The experimenter then removed the pictures from the child's view by the experimenter and children were instructed to count up to 50 for a period of 30 seconds. This served as a buffer clearing task to control for any recency or primacy effects. Children were then asked to recall as many items as possible by verbal report. Subjects recall was recorded with a voice recorder so that clustering at retrieval could be computed afterwards. After the first silent period of 10 seconds in which the child did not report any additional pictures, the experimenter asked whether there were any more pictures that the child could remember. After another continued silence of 15 seconds, the task was ended.

The second sort-recall task was identical with the exception that, before the start of this task, children were told that it might be easier to remember the pictures if they are organized in groups of pictures that belong together. This instruction is considered nondirective because no hints were given as to the perceptual or semantic categories into which pictures could be grouped. At the end of the second 
sort-recall task, children were checked for colour-blindness by asking them to label the four colours that were used.

At the second measurement point, all children also performed a transfer-sortrecall task (see introduction). In this task, children received the same neutral instructions as in the standard task (see above). This was done to examine if PD and consistently strategic children were able to generalize strategy use to a sort-recall task with new categories and pictures.

Table 2. Time schedule showing which tasks were administered at time point 1 and time point 2 .

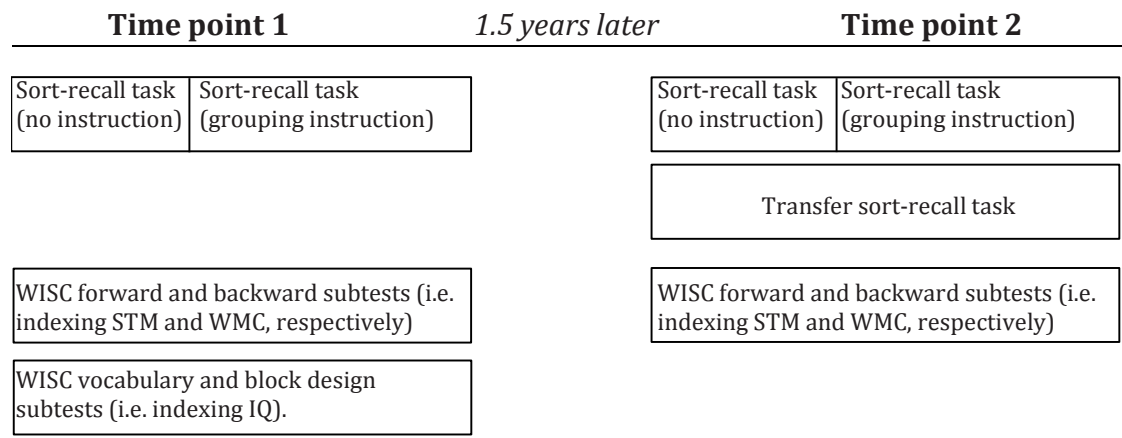

\section{Tasks}

\section{Sort-recall task}

The material for the sort-recall task comprised two stimulus sets of 12 black and white line drawings of objects, printed on $6 \mathrm{~cm} \times 5 \mathrm{~cm}$ cards. The borders of the cards $(0.2 \mathrm{~cm})$ were printed in one of four colours (i.e. green, blue, brown, grey). The objects in each set could be grouped according to semantic category (four objects from three distinct categories; see Table 3) and/or by color. The color manipulation was already included in our initial study to investigate if children group information on the basis of color (Schleepen \& Jonkman, 2012). This was not the case, but the color manipulation was still included in the current study to keep the material perceptually similar as the sort-recall task administered at time point 1 . For each stimulus set, different pictures of objects (from the same semantic categories) were used and the two stimulus sets were counterbalanced across the two sort-recall tasks and across age groups.

The choice of line drawings in the two stimulus sets was based on several considerations. First, semantic categories of stimuli were selected that were common to children within the age range of 6-12 years-old. This was undertaken based on familiarity rates for 5- and 6-year-old-children collected by Cycowicz et al. 
(1997). This procedure yielded highly and equally familiar objects from the three semantic categories. Second, only object stimuli were included that consisted of 37 letter words. This restriction ensured that differences in recall performance could not be attributed to the fact that short item-words are better recalled than long-item words (Neath, Bireta, \& Surprenant, 2003). Finally, the least-associative items within each category were selected to constitute the two stimulus sets. This selection was accomplished by means of a pilot study with adults in which all possible combinations of pairings of two pictures from the same semantic category were presented to a group of 13 adults. These adults rated the level of association between the items on a 10-point scale ( $1=$ low associative and $10=$ high associative). Mean association scores for all pairs per category fell between 2.3 and 2.7 (SD between 1.5 and 1.9). Adults were instructed that the extent of associativeness could be based on different features and that the ratings should be based on their first impressions. The rationale behind including adults in this pilot was hat if adults, with their elaborate knowledge bases, were to rate item pairs as having a low semantic association, one can assume this would be the case for children.

Since children had to remember the same two sets of pictures in the sortrecall tasks at the first and second time point, it was checked whether children perhaps still remembered some words by asking them to report any pictures they might remember from 1,5 years earlier. Twenty-three of the 26 children aged 8-9 years and 20 of the 25 children aged 10-12 years could not remember any pictures from time point 1 . On the basis of these findings we conclude that practice effects could not explain possible longitudinal effects.

\section{Transfer sort-recall task}

This task was similar as the sort-recall tasks described above with the exception that this task comprised different pictures of different semantic categories (see Table 3). Also, children did not receive a grouping prompt; this was done to study if children were able to spontaneously apply the semantic grouping strategy in the transfer task (i.e. instructions were similar as in the standard task; see above). Familiarity rates and word-length were more or less comparable between the pictures in the transfer sort-recall task and the pictures in the standard and instruction task. In addition, all pictures were also lowly associated with each other to study deliberate memory strategy use.

\section{Short-term memory and working memory tasks}

The digit-span forward and digit-span backward tests, adapted from the Wechsler Intelligence Scale for Children (WISC-III, Dutch version), were used as measures of STM and WM capacity, respectively. Backwards digit recall has been shown to be a 
measure of WM in children (Gathercole, Pickering, Ambridge, \& Wearing, 2004; St Clair-Thompson, 2010).

Table 3. Stimulus items per category in the two sort-recall tasks and in the transfer sort- recall task

\begin{tabular}{|c|c|c|c|c|}
\hline $\begin{array}{l}\text { Semantic } \\
\text { category }\end{array}$ & Standard task & Instruction task & $\begin{array}{l}\text { Semantic } \\
\text { category }\end{array}$ & Transfer task \\
\hline \multirow[t]{4}{*}{ Fruit } & Peer (Pear) & Ananas (Pineapple) & Tools & Liniaal (Ruler) \\
\hline & Aardbei (Strawberry) & Kers (Cherry) & & Tang (Shrew) \\
\hline & Citroen (Lemon) & Meloen (Melon) & & Zaag (Saw) \\
\hline & Druiven (Grapes) & Appel (Apple) & & Schroevedraaier (Screwdriver) \\
\hline \multirow[t]{4}{*}{ Animals } & Dolfijn (Dolphin) & Kat (Cat) & Vegetables & Paprika (Paprika) \\
\hline & Hond (Dog) & Olifant (Elephant) & & Ui (Union) \\
\hline & Vogel (Bird) & Koe (Cow) & & Mais (Corn) \\
\hline & Aap (Monkey) & Zwaan (Swan) & & Champignon (Agaric) \\
\hline \multirow[t]{4}{*}{ Clothes } & Jas (Coat) & Hoed (Hat) & Vehicles & Fiets (Bicycle) \\
\hline & Rok (Skirt) & Broek (Trousers) & & Auto (Car) \\
\hline & Das (Tie) & Want (Glove) & & Helicopter (Helicopter) \\
\hline & Sok (Sock) & Jurk (Dress) & & Zeilboot (Sailboot) \\
\hline
\end{tabular}

\section{Scoring strategy use}

The procedures for scoring strategy use were similar to those used in most prior research. Adjusted Ratio of Clustering (ARC) scores (Roenker, Thompson, \& Brown, 1971) were used to assess the amount of color-or semantic sorting during study and clustering at retrieval. The ARC score does not vary systematically with amount recalled (Murphy, 1979) making it an appropriate measure of strategy use when different levels of recall are expected for different groups, as in the present study. In the present study two types of clustering (ARC) scores are calculated. First, the sorting ARC score that reflects strategy use during the study phase of the task and reflects the extent to which pictures from the same color or semantic category are sorted in groups above a certain chance level. Second, we calculated an ARC retrieval clustering score by counting the words belonging to the same semantic category that were recalled in successive order above a certain chance level during the retrieval phase of the task. ARC scores vary between - 1 and 1 , with a score of 1 reflecting perfect sorting or clustering, a score of zero indicating sorting or clustering at chance level, and a score below zero representing sorting or clustering below chance level. In calculating the ARC sorting score, a repetition was counted if two pictures of the same semantic category or color were laid above, below or next to each other. Intrusions and perseverations were not included in calculating the ARC retrieval scores. Following Coyle and Bjorklund (1997), sorting 
during study or clustering at retrieval was considered to be meaningful (i.e. children were considered strategic) when clustering scores were equal to or greater than .5. An ARC score of .5 reflects a value of slightly more than one standard deviation greater than sorting or retrieval clustering expected by chance.

\section{Results}

Because of uninterpretable ARC sorting and retrieval clustering scores for the color dimension in all age groups (ARC score <.5), all analyses reported below only focused on semantic sorting during study and semantic clustering at retrieval. Table 4 represents mean clustering-ARC scores for sorting during study, clustering at retrieval and mean recall scores as a function of age, instruction condition and time.

\section{Longitudinal development of semantic grouping strategies}

To investigate 1) if 6- to 7-year-old children first pass through the MD phase before they are PD when they are 8- to 9-years-old and 2) if 8- to 9-year-old children first pass through the PD phase before they spontaneously and successfully use the semantic strategy at age 10 - to 12 , semantic sorting during study, semantic clustering at retrieval and recall scores were analyzed by separate instruction (2) x time (2) ANOVA's per age group (i.e. 6-7 and 8-9-year-olds at Time 1). Significant main age effects were further analyzed with bonferroni-corrected post-hoc group comparisons.

Sorting during study. For both 6-7-year-old and 8-9 year-old children, a main effects of instruction was found; $F(1,25),=29.1, p<.0001, \eta 2 p=.54$ and $F(1,24)$, $=33.4, p<.00001, \eta 2 p=.58$, respectively. For both groups, these main effects indicate that ARC sorting scores were significantly higher after than before instruction. The ANOVA analysis also revealed a main time effect in 6-7 year-olds, $F(1,25)=$ $14.1, p<.01, \eta 2 \mathrm{p}=.36$, and a trend-significant main time effect in 8-9 year-olds, $F(1,24)=3.4, p=.079 \eta 2 \mathrm{p}=.12$; in both groups semantic sorting was higher at time 2 than at time 1 . To conclude whether semantic sorting scores were meaningful $(A R C \geq .5)$ or not in both age groups (see Table 3), also mean group ARC values should be considered when interpreting these main effects. The 6-7-year-old children showed meaningless $(\mathrm{ARC}<.5)$ semantic sorting scores at time 1 both before and after prompting. At time two, 1,5 years later (at the age of 8-9) these children still had meaningless sorting scores before prompting, but now prompting did lead to meaningful semantic sorting. The children that were 8-9-years-old at time 1 had meaningless sorting scores before instruction at both time points, whereas 
prompting led to almost meaningful (.47) sorting at time 1 and clearly above chance sorting (.87) at time 2 .

Clustering at retrieval. Also for clustering at retrieval, both 6-7 and 8-9-yearold children showed a main effect of instruction; $F(1,25),=12.6, p<.01, \eta 2 p=.34$, and $F(1,24)$, = 11.1, $p<.01, \eta 2 p=.32$, respectively. Also a main effects of time was found for both groups; $F(1,25)=9.5, p<.01, \eta 2 p=.28$, in $6-7$ year olds and $F(1,24)$ $=10.6, p<.01, \eta 2 \mathrm{p}=.31$, in 8-9 year-olds. For both groups, these main effects indicate higher semantic ARC retrieval scores after than before instruction, and higher semantic clustering during retrieval at time 2 than time 1 . The mean retrieval clustering ARC values (see Table 3) indicate that 6-7- year-olds had meaningless clustering retrieval scores at Time 1, both before and after prompting. At time 2 when these children were 8-9 years old they still showed meaningless retrieval clustering before prompting, but prompting now led to meaningful semantic clustering. At time point 1, 8-9-old children had meaningless clustering retrieval scores before prompting while these scores were meaningful $(\mathrm{ARC}=.50)$ after prompting. After 1,5 years when these children were 10-12 years of age, semantic clustering scores before prompting were at the same level $(\mathrm{ARC}=.50)$ as after prompting at time 1 , but further increased to .73 after prompting.

Recall. For recall performance, a main effect of time was found in both age groups, indicating that both 6-7 and 8-9 year-old children had higher recall scores at Time 2 than at Time $1, F(1,25),=25.2, p<.0001, \eta 2 p=.50$, and $F(1,24)=34.2$, $p<.0001, \eta 2 p=.59$, respectively). We established whether there were any intrusions from prior lists that might have served as a retrieval cue for items of the current list. In total four intrusions from a prior list occurred (who were made by four different children), but only one intrusion was followed by an item from the same semantic category.

\section{Relation between strategy use and recall}

To determine at what age children were mediation or production deficient or consistently strategic and how this developed, Pearson's correlations were computed between sorting during study, clustering at retrieval and recall separately for the two age groups per instruction condition at the two time points. See Table 4 for all correlations. Significant positive correlations were found between ARC-sorting and recall scores and ARC-retrieval and recall scores in 8-9-year-olds at Time 1 after prompting, as well as after 1,5 years at the age of 10-12 years both before and after prompting. In the 6-7-year-olds these correlations reached significance only at Time 2 before prompting (i.e., when they were 8- to 9-years), but because of mean group ARC values that are smaller than $<.5$ these correlations are not further interpreted. 
Summarizing the longitudinal data, 6-7-year-olds had meaningless $(\mathrm{ARC}<.5)$ semantic sorting and retrieval clustering scores in both instruction conditions at time 1 , which is consistent with a MD. After 1.5 years when these children were 8-9 years, sorting and retrieval organization scores in the task without prompting were increased and correlated with recall but were still meaningless. After prompting they did show meaningful sorting and retrieval organization, and correlations of $r=.32$ and $r=.27$, were found for sorting-recall and retrieval clusteringrecall, respectively. Although these latter correlations did not reach significance, the fact that significant correlations of both scores with recall were present in the transfer task allows for the conclusion that these children were PD at time 2. The 8-9-year-olds also had below chance semantic sorting and retrieval clustering scores at time 1 before prompting, but the prompt at time 1 led to meaningful sorting and retrieval clustering scores that were related to increased recall levels. This pattern at time 1 indicates a PD for both strategies in children aged 8-9 years. After 1.5 years when they were 10-12 years-old, they were still PD with respect to the sorting strategy, but had become consistently strategic with respect to retrieval clustering (ARC $\geq .5$ ); retrieval clustering scores related to recall both without and with prompting at time 2).

Table 4. Means and Standard Deviations (between brackets) in sorting, retrieval clustering scores and recall by age group, task and measurement point.

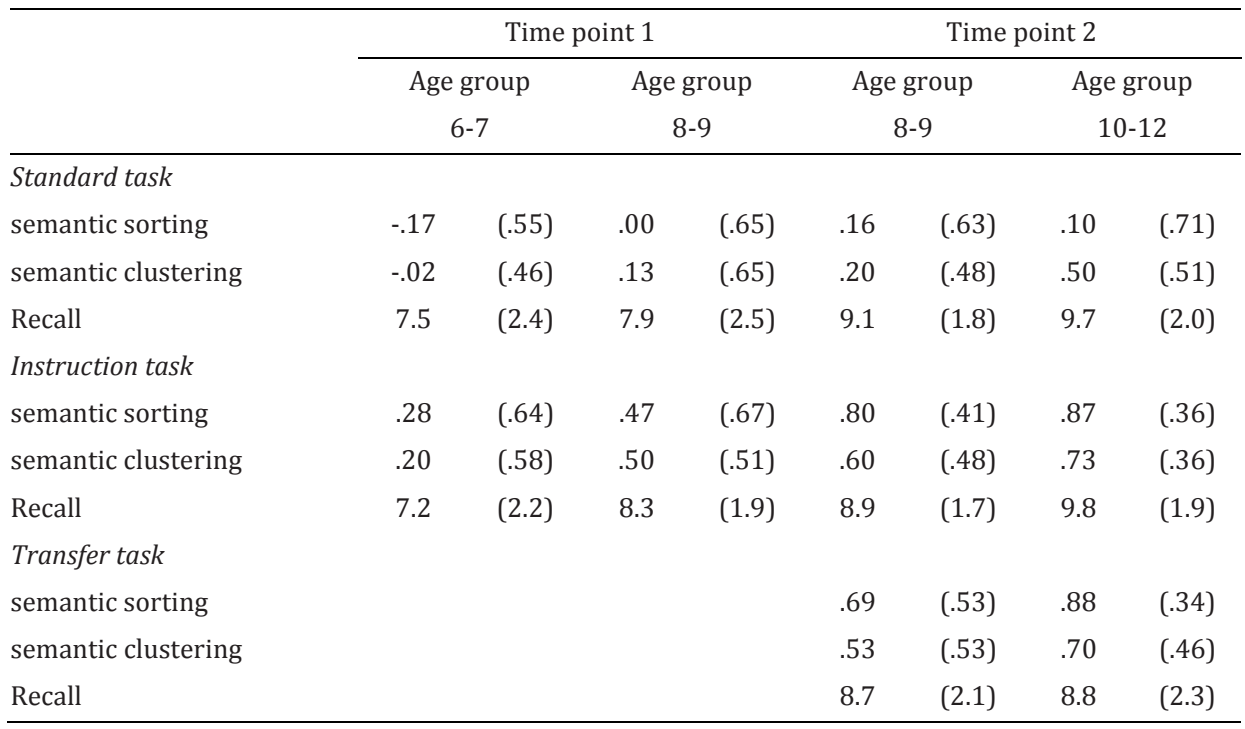

Note: the 8-9 year-olds at time point 2 were 6-7 years-old at time 1, and the 10-12 year-olds at time point 2 were $8-9$ years-old at time 1 . 
Table 5. Pearson's correlations between sorting during study, clustering at retrieval and recall by age group, task and measurement point.

\begin{tabular}{|c|c|c|c|c|}
\hline & \multicolumn{2}{|c|}{ Time point 1} & \multicolumn{2}{|c|}{ Time point 2} \\
\hline & Age group 1 & Age group 2 & Age group 1 & Age group 2 \\
\hline & $6-7$ & $8-9$ & $8-9$ & $10-12$ \\
\hline \multicolumn{5}{|l|}{ standard task } \\
\hline sorting-clustering & .02 & $.57^{* *}$ & $.75^{* *}$ & $.59^{* *}$ \\
\hline sorting-recall & -0.2 & .13 & $.52^{* *}$ & $.50^{*}$ \\
\hline clustering-recall & $-.47^{*}$ & .28 & $.64^{* *}$ & $.44^{*}$ \\
\hline \multicolumn{5}{|l|}{ instruction task } \\
\hline sorting-clustering & $.62^{* *}$ & $.53^{* *}$ & .25 & $.45^{*}$ \\
\hline sorting-recall & .36 & $.44^{*}$ & .32 & $.61^{* *}$ \\
\hline clustering-recall & .34 & $.44^{*}$ & .27 & $.49^{*}$ \\
\hline
\end{tabular}

Note: the 8-9 year-olds at time point 2 were 6-7 years-old at time 1 , and the 10-12 year-olds at time point 2 were $8-9$ years-old at time 1 .

$* p<.05$

${ }^{* *} p<01$

\section{Strategy Transfer}

To answer our research question whether production deficient children were able to transfer their recently acquired semantic grouping skills and differed in this ability from consistent strategy users, children were first classified as production deficient or consistent strategy users on the basis of their individual ARC scores in the sort-recall task administered at time point 2 . A child was classified as production deficient when ARC scores were $<.5$ in the task without instruction and $\geq .5$ in the task with instruction. A child was considered as consistent strategy user when ARC scores were $\geq .5$ both in the task without and with instruction. This division in production deficient children and consistent strategy users was done separately for the sorting and clustering retrieval strategy. To examine possible differences in (the success of) strategy transfer between production deficient children and consistent strategy users, independent T-tests were run for sorting scores, retrieval clustering scores and recall scores in the transfer task administered at time point 2. Also, correlations were computed between the strategy measures (ARC sorting and retrieval scores) and recall performance to evaluate if production deficient children were able to successfully apply the semantic grouping strategy in the transfer task. See Table 6 for mean ARC scores for sorting during study, clustering at retrieval and mean recall scores in the transfer-sort recall task in production deficient children and consistent strategy users for the sorting and retrieval clustering strategy. 
For the sorting strategy, 27 children were classified as production deficient and 18 children as consistent strategy user (the remaining children could not be placed in either group). In the standard task at time 2 (on the basis of which children were classified as production deficient or consistent strategy user), recall performance differed significantly between production deficient children and consistent strategy users in the task without instruction $[t(43)=-3.4, p<.01]$, but not in the task with instruction $[t(43)=-.04, p>.1]$, indicating that both groups were equally successful in applying the semantic sorting strategy after having received the prompt. The analyses in the transfer task however revealed that whereas ARC sorting and ARC retrieval scores did not significantly differ between production deficient children and consistent strategy users (who both had ARC scores $\geq .5$ ) $[t(43)=-.51$, $p>.1$, and, $t(43)=-.75, p>.1$, respectively], consistent strategy users had higher recall scores than production deficient children [ $t(43)=-2.1, p<.05]$.

For the clustering retrieval strategy, 19 children were classified as production deficient and 18 as consistent strategy user (the remaining children could not be placed in either group). Results in the standard task showed the expected pattern of recall performance differing significantly between production deficient children and consistent strategy users in the task without instruction $[t,(35)=-2.5, p<.05]$, but not in the task with instruction $[t,(35)=-.70, p>.1]$, indicating that both groups were equally successful in using the semantic retrieval strategy in the task after prompting. In the transfer task however, consistent strategy users had significantly higher ARC sorting $[t,(35)=-2.1, p<.05]$, ARC retrieval $[t,(35)=-3.0, p<.01]$ and recall scores $[t,(35)=-3.3, p<.01]$ compared to production deficient children. This indicates that, although both production deficient children and consistent strategy users had meaningful (>.5) ARC sorting and ARC retrieval clustering scores (see Table 6), strategy use in the transfer task was higher and more successful in consistent strategy users children than in production deficient children.

Regarding overlap of the strategy subgroups in sorting and retrieval clustering, 13 children were production deficient both for the sorting and clustering retrieval measure, and also 13 children were consistent strategy users both for the sorting and clustering retrieval measure. There were no significant differences in age, gender or IQ between production deficient children and consistent strategy users (neither for sorting nor for retrieval clustering), except that consistent clusterers were significantly older than production deficient clusterers (see Table 6 for mean age, gender distribution and mean IQ scores in all strategy subgroups).

See Table 6 for the correlations between sorting during study, clustering at retrieval and recall in all 4 strategy groups (production deficient sorters, consistent sorters, production deficient clusterers, consistent clusterers). Summarizing these correlations, production deficient sorters and production deficient clusterers had significant correlations between sorting scores, clustering retrieval scores, and recall performance. These correlations indicate that they successfully 
used the semantic grouping strategy in the transfer task. Most correlations were nonsignificant in consistent strategy users, but this is most likely due to ceiling effects. For example, more than $60 \%$ of the consistent strategy users (both for the sorting and clustering retrieval strategy) had a recall score of 11 or 12, and maximum ARC sorting and retrieval scores were also obtained by large percentages of consistent strategy users (ranging from 70\%-90\% of the children that had the maximum ARC score of 1 ).

Table 6. Upper panel: Mean age, gender distribution and mean IQ scores of all strategy subgroups. Middle panel: Means and Standard Deviations (between brackets) in sorting, retrieval clustering scores and recall by strategy subgroup in the transfer task. Lower panel: Pearson's correlations between sorting during study, clustering at retrieval and recall by strategy subgroup in the transfer task (see results section for how the strategy subgroups were formed).

\begin{tabular}{lcccc}
\hline & \multicolumn{2}{c}{ Strategy group for sorting } & \multicolumn{2}{c}{ Strategy group for clustering } \\
\hline Descriptives & PD & CSU & PD & CSU \\
Age & $9.5(1.3)$ & $9.5(1.4)$ & $9.0(1.1)$ a & $10.1(1.3)$ a \\
gender (\%female) & 48.2 & 50.0 & 47.4 & 55.6 \\
IQ & $106(11.2)$ & $111(14.0)$ & $109.7(13.5)$ & $108.4(11.1)$ \\
Means & & & & $.74(.52)$ \\
semantic sorting & $.89(.31)$ & $.94(.27)$ & $.51(.47)$ & $.88(.24)$ \\
semantic clustering & $.71(.38)$ & $.79(.30)$ & $8.3(2.2)$ & $10.4(1.7)$ \\
recall & $9.3(1.4)$ & $10.3(1.6)$ & & -.23 \\
Correlations & & & $.77^{* *}$ & .39 \\
sorting-clustering & $.69^{* *}$ & $.46^{* * *}$ & $.79^{* *}$ & .33 \\
sorting-recall & $.35^{* * *}$ &. .11 & $.71^{* *}$ & .33 \\
clustering-recall & $.44^{*}$ & & & \\
\hline
\end{tabular}

Note: $\mathrm{PD}=$ production deficient, $\mathrm{CSU}$ = consistent strategy users. ${ }^{\mathrm{a} C S U}$ children were significantly older than PD children

\section{Role of STM/WM in semantic strategy use}

To examine if a child's STM and/or WMC at time point 1 predicted recall performance in children who used the sorting or clustering retrieval strategy at time point 2, the following steps were performed (this was done separately for the task without and with a grouping instruction). First, subgroups of children that did or did not apply the sorting or clustering retrieval strategy were created on the basis of having an ARC score $\geq$ or $<.5$ (according to recommendations by Coyle \& Bjorklund, 1997). Second, in strategic and nonstrategic groups correlations were computed between forward and backward digit span scores measured at time 1 (as a measure of STM and WMC, respectively) and recall scores at time 2. When 
significant correlations were found, regression analysis was done to test whether STM/WMC explained significant variance in recall performance.

\section{Standard task (without prompting)}

Regarding the sorting strategy, there were 33 nonstrategic and 18 strategic children. Results of the correlation analysis showed that in both strategic and nonstrategic sorters, neither STM nor WMC scores at time 1 correlated with recall scores at time 2 (all $p s>1$ ). For the clustering retrieval strategy, 28 children were classified as nonstrategic and 23 as strategic. It was found that in nonstrategic clusterers, digit span backward scores at time 1 correlated positively with recall scores at time $2(r=.36, p=.06)$ and regression analysis revealed that time 1 WMC contributed trend-significantly to time 2 recall performance (i.e. explained $13 \%$ of the variance, $p=.06$ ). No significant correlations were found in strategic clusterers $(p s>1)$.

\section{Instruction task}

For the sorting strategy, 6 children were classified as nonstrategic and 45 children were classified as strategic. Given the low number of nonstrategic sorters, no further statistical analysis could be carried out in this group. In strategic sorters, time 1 digit span backward scores were positively correlated with time 2 recall scores $(r=.26, p=.085)$, but regression analysis demonstrated that digit span backward did not explain significant variance in recall performance $(p>1)$. Regarding the clustering retrieval strategy, there were 17 nonstrategic children and 37 strategic children. No significant correlations were found in these groups ( $p s>.1)$.

\section{Discussion}

The present longitudinal study had three goals: 1) to investigate if 6-7-year-olds first pass through to MD phase before entering the PD phase at 8-9-years of age, and if 8-9-year-olds first pass through the PD phase before they are able to spontaneously and successfully use the semantic grouping strategy at 10- to 12 years of age, 2) to examine whether children that progressed to the PD phase after having received a single indirective grouping prompt, demonstrate strategy transfer when not prompted and 3) to examine if STM/WMC predicts semantic strategy use longitudinally. These three goals will be separately discussed below. 


\section{Longitudinal development of semantic grouping strategies}

The current longitudinal study, that included a subset of children from our earlier cross-sectional study (Schleepen \& Jonkman, 2012), more or less replicates the developmental progression of semantic grouping strategy use that we found in this earlier study.

The present longitudinal data revealed that children that were mediation deficient at age 6-7 years progressed to being production deficient, i.e. being able to use the strategy after prompting, at the age of 8-9 years. This was the case for both the sorting and clustering retrieval strategy as was shown by ARC sorting and retrieval clustering scores $\geq .5$ only after prompting at time 2 . In 8-9 year-old children, the sorting and clustering retrieval strategy each showed a different succession of developmental phases. With respect to the clustering retrieval strategy it was found that 8-9 year-old production deficient children (who at this age only had clustering scores above chance leading to higher recall after prompting) were consistently strategy at the age of 10-12. This was shown by above-chance clustering and significant clustering-recall relations in the sort-recall task without and with prompting. Thus, after 1,5 years these children still successfully applied the clustering strategy during the retrieval of pictures, now also without prompting. Application of the sorting strategy showed a different longitudinal developmental pattern; the finding that semantic sorting scores were only meaningful after prompting at both time points indicates that 8-9 year-old children that were production deficient with respect to the sorting strategy, were still production deficient with respect to sorting 1,5 years later at the age of 10-12 years. Thus, although the prompt at time 1 led to successful use of the sorting strategy in 8-9 year-olds, these children still needed prompting to apply this strategy successfully when they had reached the age of 10-12 years.

The reason why 10-12 year-olds were still production deficient (i.e. at time 2) for the sorting strategy might be because they now covertly applied the semantic grouping strategy during encoding, thus without showing signs of overtly sorting the pictures in semantic groups. This is consistent with findings by Schwenck et al. (2009) reporting that among 4-8 year-olds, older children more frequently used covert strategies during selective learning, whereas younger children tended to use more overt strategies such as naming or pointing. It has to be noted that although sorting was again meaningful after prompting at time 2, this might merely indicate that the prompt triggered the children again to sort the pictures (but this was not more successful than when items were not sorted as was the case before prompting; recall before prompting was 9.7 and recall after prompting was 9.8).

Importantly, whereas different lists were used for no-instruction and instruction sort-recall tasks to prevent effects of learning the pictures within one session, the same two lists were used at time 2 based on the assumption that no pictures 
would be remembered after 1,5 years. We verified that $88.5 \%$ of the $8-9$ and $80 \%$ of the 10-12 year-olds did not remember any of the pictures they had studied 1,5 years earlier at the beginning of measurement 2 . Also, excluding the children that did remember any pictures from time 1 from the statistical analyses did not change the results. This makes it highly unlikely that practice effects influenced our developmental findings.

The current longitudinal study contributes to the already existing longitudinal research on memory strategy development by investigating, for the first time, the developmental transition between the mediation deficiency and production deficiency phase before children become consistent strategy users. Prior longitudinal studies (Kron-Sperl, et al., 2008; Schlagmuller \& Schneider, 2002; Schneider, et al., 2009; Schneider, et al., 2004) investigated the occurrence of utilization deficiencies, but these studies could not always replicate the presence of this developmental phase as it was reported in cross-sectional studies (Bjorklund \& Coyle, 1995; Bjorklund, et al., 1997; Schwenck, et al., 2009). The reason for this may be that the criteria on the basis of which children were defined as utilization deficient differed between these studies. But there were also other methodological differences between these studies (e.g. differences in the measure used to assess strategy use; sorting vs. retrieval clustering) (Schwenck, Bjorklund, \& Schneider, 2007). The present longitudinal replication of the mediation deficiency and production deficiency phase suggests that these phases truly exist in children's semantic strategy acquisition.

\section{Strategy transfer}

The second goal of the current study was to investigate if production deficient children showed strategy transfer to a new task, and if strategy use and its effects on recall performance in this transfer task differed between production deficient children and consistent strategy users. This was done by comparing strategy use (i.e. sorting and retrieval clustering scores) and recall performance in the transfer task between production deficient children and consistent strategy users. The classification in production deficient children and consistent strategy users was done on the basis of individual ARC scores obtained in the sort-recalls task administered at time point 2 , separately for the sorting and clustering retrieval strategy. The terms production deficient sorters and production deficient clusterers, that will be used hereafter, thus refer to children that only successfully used the semantic sorting or clustering retrieval strategy, after having received the prompt in the sort-recall task administered at time point 2. Consistent strategy users are children that successfully applied the semantic grouping strategy both in the task without and with prompting at time 2 . The prompt was a single non-directive grouping 
prompt in which the to-group-on categories were not mentioned. The transfer task was a sort-recall task consisting of new pictures and new semantic categories.

It was found that both production deficient sorters and production deficient clusterers were able to generalize the semantic sorting as well as clustering retrieval strategy to the near transfer sort-recall task, as was shown by ARC scores 2.5. Moreover, significant positive correlations between strategy use (ARC scores) and recall performance in both production deficient groups indicate that they successfully used the semantic sorting and clustering retrieval strategy in the transfer task. Production deficient sorters and production deficient clusterers differed however in the extent to which their semantic strategy use in the transfer task was comparable to that of CSU children. That is, while production deficient sorters showed comparable transfer of both the sorting and clustering retrieval strategy as consistent strategy users for sorting (equal ARC scores), production deficient clusterers generalized these strategies to a lesser extent to the transfer task than consistent strategy users for clustering (had lower ARC sorting and clustering retrieval scores). Yet, both production deficient sorters and production deficient clusterers had significantly lower levels of recall in the transfer task than their respective consistent strategy users comparison groups. Thus, although both production deficient subgroups reached a comparable level of success of semantic strategy use as the consistent strategy users subgroups after having received the prompt at time 2 , and showed transfer of strategy use to a new sort-recall task, strategies were used more frequently and led to higher recall in the transfer task in children that were consistently strategic.

The reason that transfer was still less successful in production deficient children might be because the metacognitive skills of these children, i.e. recognizing that strategy use leads to improved memory performance, are not yet fully developed (at least not as much as those of consistent strategy users children). Findings by Melot (1998) support this idea, demonstrating that among 6- to 9-year-old children, those children that better understood that effective strategy use can positively influence their recall performance, were better able to also use this strategy on the post-test. Further, the current finding that transfer of semantic grouping strategies was better in production deficient sorters than production deficient clusterers might be because children see their grouping if they use the sorting strategy, which is thought to increase a child's metacognitive awareness (Schwenck, et al., 2009). In this way, children can more easily relate the application of the strategy to improved memory performance, thereby stimulating later use of grouping strategies.

As far as we know, only one other study investigated strategy transfer in production deficient children separately for the sorting and clustering retrieval measure (Schwenck, et al., 2009). In this study it was reported that neither production deficient sorters nor production deficient clusterers were able to transfer their 
semantic grouping skills, even though these children became production deficient after having received rather extensive training in using semantic grouping strategies. The absence of strategy transfer in the study of Schwenck et al. (2009) might be explained by the fact that the PD children in their study were younger (between 4-8 years of age) than in our study (8-12 years of age at time point 2 when strategy transfer was studied). The current findings demonstrate that children of 8 years and older who became production deficient after only having received a general grouping prompt, show successful strategy transfer when not prompted, although their performance in the transfer task is still inferior compared to that of consistent strategy users. If more extensive training would have been provided, for instance a training similar to the one provided by Schwenck et al. (2009) and also including training of metacognitive skills, production deficient children would possibly have shown equal benefit of application of the strategies as consistent strategy users. This hypothesis should be investigated in future studies.

\section{Role of WMC in semantic grouping strategy use}

Because our previous cross-sectional study (Schleepen \& Jonkman, 2012) showed a relation between WMC and successful use of the semantic grouping strategy, a third goal was to examine this relationship in a longitudinal design. Specifically, we examined whether WMC at time 1 (inferred from digit span backward scores) predicted recall performance in children who did or did not use the sorting or clustering retrieval strategy at time 2 . There only was a trend-significant correlation showing that WM scores at time 1 predicted recall scores in nonstrategic clusterers 1,5 years later. However, no statistical evidence was found for a predictive role of WMC in successfully using the sorting or clustering retrieval strategy 1,5 years later. Several reasons may be given for why we did not find a longitudinal relation between WMC and semantic grouping strategy use. First, it could be argued that a developmental shift took place in the factors contributing to successful use of this strategy. That is, whereas children might have relied on WM-resources during strategy implementation at time point 1 , during the 1.5 year transition period other factors not measured in this study (e.g. a child's knowledge base; Bjorklund, 1987) might have become to play a more important role in the development of the semantic grouping strategy. Second, while the backward digit-span test has been shown to have adequate internal consistency (Waters \& Caplan, 2003) and thus can be considered a suitable measure of WM in children (St Clair-Thompson, 2010), it may be that using a composite score reflecting performance on several (partly different) WM tests might have better predictive value than the score on a single WM-test. Future longitudinal research that addresses the WMC-semantic strategy use link should take these points into consideration. 


\section{References}

Achenbach, T. M. (1991). Manual for the child behavior checklist/4-18 Burlington: University of Vermont, Departement of Psychiatry.

Baddeley. (2000). The episodic buffer: a new component of working memory? Trends in Cognitive Sciences, 4, 417-423.

Bauer, R. H. (1977). Memory processes in children with learning disabilities: evidence for deficient rehearsal. Journal of Experimental Child Psychology, 24, 415-430.

Bjorklund, D. F. (1987). How age changes in knowledge base contribute to the development of children's memory: An interpretive review Developmental Review, 7, 93-130.

Bjorklund, D. F., \& Coyle, T. R. (1995). Utilization deficiences in the development of memory strategies In F. E. Weinert \& W. Schneider (Eds.), Memory performance and competencies: Issues in growth and development (pp. 161-180). Mahwah, NJ: Erlbaum.

Bjorklund, D. F., \& de Marchena, M. R. (1984). Developmental shifts in the basis of organization in memory: The role of associative versus categorical relatedness in children's free recall. Child Development, 55, 952-962.

Bjorklund, D. F., \& Jacobs, J. W. (1985). Associative and categorical processes in children's memory: The role of automaticity in the development of organization in free recall. Journal of Experimental Child Psychology, 39, 599-617.

Bjorklund, D. F., Miller, P. H., Coyle, T. R., \& Slawinski, J. L. (1997). Instructing children to use memory strategies: Evidence of utilization deficiencies in memory training studies. Developmental Review, 17, 411-441.

Coyle, T. R., \& Bjorklund, D. F. (1997). Age differences in, and consequences of, multiple- and variablestrategy use on a multitrial sort-recall task. Developmental Psychology, 33, 372-380.

Cycowicz, Y. M., Friedman, D., Rothstein, M., \& Snodgrass, J. G. (1997). Picture naming by young children: Norms for name agreement, familiarity, and visual complexity. Journal of Experimental Child Psychology, 65, 171-237.

Flavell, J. H. (1970). Developmental studies of mediated memory. In H. W. Reese \& L. P. Lipsitt (Eds.), Advances in child development and behavior (Vol. 5, pp. 181-211). New York: Academic.

Gathercole, S. E., Pickering, S. J., Ambridge, B., \& Wearing, H. (2004). The structure of working memory from 4 to 15 years of age. Developmental Psychology, 40, 177-190.

Hollingshead, A. B. (1975). Four factor index of social status. New Haven, CT: Yale University of Department of Sociology.

Jeyakumar, S. L. E., Warinner, E. M., Raval, V. V., \& Ahmad, S. A. (2004). Balancing the need for reliability and time efficiency: Short forms of the Wechsler Adult Intelligence Scale-III. Educational and Psychological Measurement, 64, 71-87.

Kron-Sperl, V., Schneider, W., \& Hasselhorn, M. (2008). The development and effectiveness of memory strategies in kindergarten and elementary school: Findings from the Wurzburg and Gottingen longitudinal memory studies. Cognitive Development, 23, 79-104.

McNamara, D. S., \& Scott, J. L. (2001). Working memory capacity and strategy use. Memory \& Cognition, $29,10-17$.

Melot, A. M. (1998). The relationship between metacognitive knowledge and metacognitive experiences: Acquisition and re-elaboration. European Journal of Psychology of Education, 13, 7589.

Murphy, M. (1979). Measure of category clustering in free recall. In R. Puff (Ed.), Memory organization and structure (pp. 51-81). New York: Academic Press.

Neath, I., Bireta, T. J., \& Surprenant, A. M. (2003). The time-based word length effect and stimulus set specificity. Psychonomic Bulletin \& Review, 10, 430-434.

Reese, H. W. (1962). Verbal mediation as a function of age level. Psychological Bulletin, 59, 502-509. 
Ringel, B. A., \& Springer, C. J. (1980). On knowing how well one is remembering: the persistence of strategy use during transfer. Journal of Experimental Child Psychology, 29, 322-333.

Roenker, D. L., Thompson, C. P., \& Brown, S. C. (1971). Comparison of measures for the estimation of clustering in free recall. Psychological Bulletin, 76, 45-48.

Rosen, V. M., \& Engle, R. W. (1997). The role of working memory capacity in retrieval. Journal of Experimental Psychology-General, 126, 211-227.

Schlagmuller, M., \& Schneider, W. (2002). The development of organizational strategies in children: evidence from a microgenetic longitudinal study. Journal of Experimental Child Psychology, 81, 298-319.

Schleepen, T. M. J., \& Jonkman, L. M. (2012). Children's use of semantic organizational strategies is mediated by working memory capacity. Cognitive Development, 27, 255-269.

Schneider, W., Kron-Sperl, V., \& Hunnerkopf, M. (2009). The development of young children's memory strategies: Evidence from the Wurzburg Longitudinal Memory Study. European Journal of Developmental Psychology, 6, 70-99.

Schneider, W., Kron, V., Hunnerkopf, M., \& Krajewski, K. (2004). The development of young children's memory strategies: First findings from the Wurzburg Longitudinal Memory Study. Journal of Experimental Child Psychology, 88, 193-209.

Schwenck, C., Bjorklund, D. F., \& Schneider, W. (2007). Factors influencing the incidence of utilization deficiencies and other patterns of recall/strategy-use relations in a strategic memory task. Child Development, 78, 1771-1787.

Schwenck, C., Bjorklund, D. F., \& Schneider, W. (2009). Developmental and individual differences in young children's use and maintenance of a selective memory strategy. Developmental Psychology, 45, 1034-1050.

Smith, E. E., Jonides, J., \& Koeppe, R. A. (1996). Dissociating verbal and spatial working memory using PET. Cerebral Cortex, 6, 11-20.

Spreen, O., Strauss, E., . (1998). A compendium of neuropsychological tests: Administration, norms, and commentary. New York: Oxford University Press.

St Clair-Thompson, H. L. (2010). Backwards digit recall: A measure of short-term memory or working memory? European Journal of Cognitive Psychology, 22, 286-297.

Waters, G.S., \& Caplan, D. (2003). The reliabity and stability of verbal working memory measures. Behavior Research Methods, Instruments \& Computers, 35, 550-564. 


\section{Chapter 5}

\section{Dissociating the effects of semantic grouping and rehearsal strategies on Event-Related Brain Potentials}




\begin{abstract}
The application of elaborative encoding strategies during learning, such as grouping items on similar semantic categories, increases the likelihood of later recall. Previous studies suggested that application of a semantic grouping strategy had modulating effects on specific ERP components. However, because in these studies subjects did not receive any explicit strategy instructions on how to encode the material, more simple strategies, like rote rehearsal, might have (partly) accounted for the reported ERP effects. To investigate this, in the current study subjects had to encode either four semantically related or unrelated pictures by respectively applying a semantic category grouping or a simple rehearsal strategy. Another goal was to investigate if maintenance of semantically grouped vs. ungrouped pictures modulated ERP-slow waves differently. At the behavioral level there was only a semantic grouping benefit in terms of faster responding on no-response trials (i.e. when the memory probe stimulus was not part of the memory set). At the neural level, during encoding semantic grouping only had specific modulatory effects on a fronto-central Late Positive Component (LPC) emerging around $650 \mathrm{~ms}$ and on left and right frontal slow wave activity during maintenance. Other ERP components (i.e. P200, N400 and late sustained potential) that were earlier related to semantic grouping encoding processes now showed stronger modulations by rehearsal than by semantic grouping. These results stress the importance of careful control of strategy use when investigating the neural correlates of elaborative encoding.
\end{abstract}




\section{Introduction}

Learning enables us to acquire the skills and knowledge to be successful in school and in life more general. The use of memory strategies is fundamental to the learning process because they aid in the encoding, maintenance and retrieval of learned information in memory. Especially when the material we need to study exceeds our memory span it is important to use memory strategies because they can reduce memory load by keeping information in mind in a more organized way. It is well known that in particular the strategies used at the time of encoding are an important determinant of how well information can be learned or later recalled (Craik \& Lockhart, 1972). Craik and Lockhart (1972) stressed that memory can be particularly improved if one elaborates on the incoming information rather than processing it in a more shallow way (e.g. on perceptual features), due to more robust memory traces aiding later recall. Elaboration is the process of attaching meaning to new information, for example by relating images, past experiences or associations from long-term memory (LTM) to the to-be-encoded information. Given that encoding strategies play a key role in whether or not material can be successfully learned, it is important to study which neural mechanisms underlie elaborative encoding. In the current study we used Event-Related Potentials (ERPs) to address this question. ERPs track the time course of neural processing at the millisecond level and might therefore increase our understanding of which cognitive sub-processes at the time of encoding lead to effective memory formation.

Recently, several studies have shown that learning to successfully use elaborative encoding strategies is dependent on capacity and requires activation of the prefrontal cortex (PFC). In an fMRI study with young and older adults, Kirchhoff, Anderson, Barch, and Jacoby (2012) investigated how learning to use three semantic encoding strategies (personal relevance, pleasantness and sentence generation) affected brain activation patterns of older adults. Only after training older adults were able to spontaneously initiate semantic encoding strategies, which increased their memory recognition performance so that it no longer differed from that of younger adult's recognition performance. The semantic strategy training increased older adult's brain activity in prefrontal and left lateral temporal regions during encoding. Miotto et al. (2006) trained young adults to use a semantic grouping strategy to encode and retrieve words in memory (i.e. group items on semantic category) which led to increased recall and semantic clustering scores, the latter reflecting the extent to which the words were grouped on semantic category during recall. These behavioural improvements co-occurred with increased activation in bilateral dorsolateral PFC and orbitofrontal cortex. Thus, these studies show that the prefrontal cortex supports the acquisition of elaborative encoding strategies. 
Moreover, several studies reported that prefrontal cortex activity was enhanced during the use of elaborative encoding strategies (vs. no strategy) even though such strategies facilitate memory performance and decrease WM-load (Bor, Cumming, Scott, \& Owen, 2004; Bor, Duncan, Wiseman, \& Owen, 2003; Bor \& Owen, 2007; Kirchhoff \& Buckner, 2006). In a series of experiments, Bor and colleagues (2003, 2004, 2007) used fMRI to investigate which brain mechanisms underlie the strategy of chunking material on the basis of different stimulus features. Irrespective of the material to which the chunking strategy was applied (e.g. verbal or nonverbal) or the specific recoding process used during chunking (e.g. mathematical or mnemonic strategies), these studies collectively show that memory performance was better on trials that allowed chunking compared to trials in which no chunking was possible. Although WM-load decreased in structured (chunking) trials, activation in fronto-parietal areas increased. In an fMRI study by Kirchhoff and Buckner (2006) subjects were presented with unrelated object pairs that interacted in a certain way (e.g. a banana in the trunk of a car) which they had to study for a later memory test. No specific strategy instructions were given about how to study the material. The strategies that were used by subjects were identified by a self-report questionnaire that was administered following a recognition test. This revealed that participants mainly used verbal elaboration and visual imagery to remember the object pairs, both strategies resulting in improved memory performance and activation of left inferior frontal and left occipitotemporal brain regions respectively.

Taken together, the above studies show that elaborative encoding relies on a prefrontal-posterior brain network. In the present study we focus on semantic grouping as elaborative encoding strategy. In specific we examined the spatiotemporal sequence of information processing underlying semantic grouping. Semantic grouping is a strategy that is used to improve verbal learning by reordering items into similar semantic categories (Mandler, 1967). It has been shown that use of this strategy leads to improved memory in individuals with compromised memory performance, such as younger children or older adults. For example, when children have reached an age at which they can spontaneously use semantic grouping strategies they often show substantial memory improvements (Bjorklund \& de Marchena, 1984; Bjorklund \& Jacobs, 1985; Schleepen \& Jonkman, 2012). Given that semantic grouping is crucial for memory and learning, it is important to gain more insight in the neural processes involved in semantic grouping, which is the main aim of the current study.

Several imaging studies already provide some information about the neural correlates underlying semantic grouping. In a study by Savage et al. (2001) participants underwent PET while performing a recall task in which they were presented with three different word lists containing either unrelated words, words that were organized in semantic categories that had to be spontaneously recognized by the 
subjects (spontaneous condition) and words that also belonged to several semantic categories but for which subjects received specific grouping instructions (directed condition). These authors reported increased activation in both the left inferior PFC and the left dorsolateral PFC in directed vs. spontaneous vs. unrelated conditions. Using a comparable paradigm, Fletcher, Shallice and Dolan (1998) reported similar relations between DLPFC activation and semantic grouping.

Due to poor temporal resolution, the above studies do however not inform us about the temporal course and duration of cognitive processes during semantic grouping. Given the speed of neural processing during memory encoding, ERPs are needed to investigate this. Although limited in number, several ERP studies give some information about the ERP components reflecting different stages of cognitive processing involved in semantic grouping. In a study by Blanchet et al. (2007) EEG was recorded while subjects were presented with sequentially presented words in three encoding conditions that differed in the degree of required semantic grouping. While in the unrelated condition the words were from different semantic categories, in both the spontaneous and guided conditions the words belonged to four different semantic categories. The two latter conditions differed from each other in that in the spontaneous condition participants were not informed about the semantic structure of the list and received no strategy instructions, while in the guided condition participants were given the names of the corresponding categories and were explicitly instructed to group the words on semantic category to aid later recall. Blanchet et al. reported several ERP components that were modulated by differences in semantic grouping demands. First, an increased P200 over predominantly fronto-central regions was observed that was largest for guided vs. spontaneous vs. unrelated conditions. Because the P200 had earlier been associated with required attentional processing during deep encoding, Blanchet et al. concluded that the largest P200 in the guided condition is caused by larger and faster attention allocation to the semantic features of the words in those conditions, since grouping categories were known beforehand. Second, a late positive component (LPC) was found between 400-800ms over centro-parietal regions. This component was largest in both the guided and spontaneous conditions compared to the unrelated condition. On the basis of others and their own findings the authors suggested that the LPC indexes voluntary associative processes involved in attempting to link words together that belong to similar semantic categories. Finally, right-frontal sustained slow wave activity was found that was increased in the spontaneous condition compared to both unrelated and guided conditions (600$1200 \mathrm{~ms}$ ) and in the spontaneous condition vs. the unrelated condition (1200$1800 \mathrm{~ms}$ ). Because the sustained right frontal slow wave was increased only in the spontaneous condition, Blanchet et al. associated this component with the degree of self-initiation involved in application of the semantic organizational strategy. Besides the study of Blanchet et al. that directly studied ERP activity during appli- 
cation of semantic grouping, other studies relevant for the present study are those that investigated which ERP components were modulated by retrieval of categoryspecific information from long-term memory (LTM). These studies reported modulations of a negative component around $400 \mathrm{~ms}$ above fronto-central, parietal and occipital electrodes and an occipital-temporal/parietal positivity (LPC) around 550 ms when specific information about object categories had to be retrieved from semantic LTM (Kiefer, 2001, 2005).

Although the above imaging and ERP studies give some insight in the neural correlates underlying semantic grouping, it cannot be excluded that in these studies subjects might have also used simple rehearsal strategies to remember information in conditions in which they did not receive any strategy instructions (e.g. in unrelated or related-spontaneous trials). Likewise, in the previously reviewed imaging studies on the neural correlates of elaborative encoding the use of more simple strategies like rehearsal to remember the structured or related material can also not be excluded. Thus, processes related to rehearsal, instead of those involved in elaborative encoding, may (partly) account for the increased activity reflected by the P2, LPC and late sustained potential in the ERP studies and prefrontal cortex in the fMRI studies in structured trials. This is an important issue since rehearsal has been shown to recruit partially overlapping brain networks (including left prefrontal cortex) as those underlying elaborative strategies (e.g. Smith \& Jonides, 1999).

Considering the above, the aim of the present study was to examine if the ERPcomponents previously associated with semantic grouping are indeed directly related to semantic grouping, or might (partly) reflect processes related to rehearsal. To achieve this goal, EEG was recorded while subjects encoded four simultaneously presented pictures of objects (S1) in two different conditions. In the semantic grouping condition these four pictures belonged to two semantic categories, and subjects were on each trial explicitly instructed to group these pictures on their corresponding semantic categories during encoding (they were not informed about the exact category names). In the rehearsal condition, the four pictures in S1 belonged to four different semantic categories (to prevent grouping on category), and here subjects received the explicit instruction to rehearse these pictures. In contrast to previous studies, this latter explicit rehearse instruction was given to experimentally control the strategies used by subjects in the unrelated condition. In this way we aimed to investigate whether the ERP-components previously associated with semantic grouping are indeed associated specifically to semantic grouping (deep encoding) or might also reflect involved rehearsal processes (shallow encoding).

Besides comparing neural activation between different strategy instruction conditions during encoding, we also investigated potential differences in neural activations during maintenance of related (semantic grouping condition) or unre- 
lated (rehearsal condition) pictures (i.e. when stimulus material was no longer visible).Therefore, following encoding, we asked subjects to maintain the four pictures in memory during a delay period until a probe occurred (S2). Memorizing related vs. unrelated pictures possibly results in distinct types of rehearsal. Keeping in memory semantically grouped pictures most likely evokes elaborative rehearsal that involves deep semantic processing of the-to-be-remembered information. In contrast, remembering the unrelated pictures will involve mere rote rehearsal. Prior neuroimaging studies reported increased activation predominantly in prefrontal brain regions when information is maintained in memory in an organized way (Prabhakaran, Narayanan, Zhao, \& Gabrieli, 2000; Wendelken, Bunge, \& Carter, 2008). ERP research showed that memory maintenance operations elicit so-called slow wave components that became more negative with increasing task difficulty, e.g. during updating vs. maintenance processes or with increasing load (Kiss, Watter, Heisz, \& Shedden, 2007; Ruchkin, Johnson, Grafman, Canoune, \& Ritter, 1992). We examined if these slow wave components are differently modulated by elaborative vs. rote rehearsal.

Based on prior research showing that deeper encoding of material leads to better memory performance (Craik \& Lockhart, 1972), we expected increased accuracy and/or decreased reaction times in semantic grouping vs. rehearsal trials. Regarding ERPs, we expected to see the first strategy effects on the P200, with higher amplitudes for semantic grouping vs. rehearsal trials based on prior findings that the P200 reflects allocation of attention that is higher when material is processed more deeply, like in semantic grouping (Blanchet et al., 2007). Furthermore, we expected to find increased N400 amplitude in semantic grouping vs. rehearsal trials, based on studies that reported effects of a semantic category manipulation on the $\mathrm{N} 400$ and suggested this is the stage at which subjects retrieve or have access to object category information in memory (Kiefer, 2001, 2005). The LPC, assumed to be related to the associative process of linking related items together (Blanchet et al., 2007), was also expected to be increased in semantic grouping vs. rehearsal trials. Finally, we do not expect to find effects on the right frontal sustained potential reported by Blanchet et al, since these effects were related to spontaneous initiation of the grouping strategy and our subjects received explicit strategy instructions in both types of trials.

For maintenance, on the basis of findings reporting increased activation in frontal brain areas when organized/grouped material is maintained in memory (Prabhakaran, et al., 2000; Wendelken, et al., 2008) and ERP studies showing that maintenance of stimulus material gives rise to ERP-slow waves (e.g. Ruchkin et al., 1992), semantic grouping trials were anticipated to elicit larger slow-wave ERP activity over frontal brain regions compared to rehearsal trials. 


\section{Methods}

Subjects

Nineteen adults, all university students, participated in this study (age range 18-25 years, mean age $=20.1$ years, 10 female) . All subjects were free from neurological or psychiatric diseases and had normal or corrected-to-normal vision as verified by self-report. Informed consent was obtained from all participants, who were rewarded with course credit for their participation. The study was approved by a local ethical committee.

\section{Procedure}

The experimental session lasted approximately 3 hours and took place in a dimly lit, sound attenuated room. Upon arrival, subjects first filled out the informed consent form, followed by attachment of the electrodes. Next, participants were seated in front of a 17-inch VGA monitor with their eyes aligned to the centre of the screen at a viewing distance of $75 \mathrm{~cm}$. Participants were instructed to minimize eye blinks and to refrain from making head or eye movements during execution of the experimental task. Before the experimental task was started, subjects performed a short practice task wherein they had to reach a predetermined performance criterion of $75 \%$ correct responses.

\section{Experimental task}

Participants performed a verbal memory task, programmed in the software package Presentation (Neurobehavioral Systems, Albany, CA; www.neurobs.com), consisting of four different stimulus conditions presented randomly intermixed within one task. There were two memory conditions that required application of either the semantic grouping or rehearsal strategy to a set of respectively related and unrelated object pictures and two control conditions in which the same stimuli were presented but that did not involve any memory requirements (only viewing instructions). Despite instructions, inspection of the grand-average ERPs and topographical maps in the control conditions however revealed late ERP modulations that should not have been present when subjects had been only passively viewing the pictures. Since the origin of this activity in the control conditions was not clear they could not function as proper control and were disregarded in further analyses. Semantic grouping and rehearsal conditions were compared with each other to answer our research questions.

In this study, the memory set consisted of four items in both experimental conditions. We deliberately kept memory load below maximum capacity (i.e. above \pm 7 items; Miller, 1956) to be able to study strategy effects on ERPs without inter- 
fering effects of load. Subjects have been found to use additional strategies when maintaining WM-loads that exceed maximum capacity (Cowan, 2001). Rypma, Berger and D'Esposito (2002) even suggested that increased activation in the PFC with higher load is the consequence of strategic changes in the way material is remembered.

The sequence of events in a trial was as follows (see Figure 1). A trial started with a $500 \mathrm{~ms}$ fixation cross, after which a $1000 \mathrm{~ms}$ cue ( $1^{\text {st }}$ cue) was presented that contained the relevant strategy instruction (e.g. rehearse, group or view) for that trial. After a $500 \mathrm{~ms}$ fixation cross the stimulus display (S1; comprising four pictures of objects) was displayed for 4500 ms around a centrally presented fixation cross and was followed by a 5000 ms retention interval. A second cue (of 1000 ms duration) then indicated what kind of decision subjects had to make on the following probe stimulus (S2). S2 appeared after a $500 \mathrm{~ms}$ fixation cross and lasted 1500 ms. Between S1 and S2 there was a centrally presented fixation cross on which subjects fixated. There was a fixed intertrial interval of $1500 \mathrm{~ms}$.

In the semantic grouping condition, the cue "group the pictures" informed participants that they had to hold S1 in memory during the S1-S2 interval by grouping the pictures on semantic category. In this condition the four pictures in S1 always belonged to two different semantic categories. There were two types of cues that could precede S2, which were randomly presented and appeared in $50 \%$ of the cases. The word "picture" required participants to decide whether S2 was or was not part of the S1 stimulus set. The word "category" indicated that subjects had to indicate whether the corresponding semantic category of S2 was similar or not to one of the semantic categories presented in the S1 stimulus set. This latter type of cue was included to assure that participants really grouped on semantic category during the encoding phase of the grouping condition and did not just rehearse the four individual S1 items. In the rehearsal condition, the cue "rehearse the pictures" indicated that S1 had to be rehearsed subvocally during the S1-S2 interval. To prevent subjects from organizing the pictures on semantic category, the four pictures in S1 in the unrelated-rehearsal condition were always from four different semantic categories. The cue preceding the memory probe S2 in the unrelated-rehearsal condition was always the word "picture", indicating that participants had to decide whether S2 was present in the S1 stimulus set or not. In the control conditions, which are not included in the analyses and results due to aforementioned reasons, S2 always consisted of two pictures. In order to keep S2 perceptually similar between control and strategy instruction conditions, in both semantic grouping and rehearsal trials S2 also always comprised two (in this case similar) pictures presented left and right from of the fixation cross (see Figure 1A). In all conditions, participants had to make a yes or no response by pressing the left- or right-hand response button using their index fingers, which was counterbalanced between participants. Participants were instructed to respond quickly, 
but never at the expense of accuracy. The experiment included a total of 270 trials presented in 9 separate blocks of 30 trials each. The control and related-grouping conditions each comprised 108 trials, while the unrelated-rehearsal condition consisted of 54 trials. This was done to keep the different types of 52 probes equal between conditions (i.e. 54 trials per type). In each block, there were 12 trials of the control and semantic grouping condition, and 6 trials of the rehearsal condition. The conditions were presented randomly within blocks. Between blocks, participants were able to take a short break.

\section{Stimuli}

S1 consisted of four pictures of objects presented in an invisible $2 \times 2$ matrix, which had a size of $13.5 \mathrm{~cm}$ horizontally by $12 \mathrm{~cm}$ vertically. The pictures $(6.5 \mathrm{by}$ $5.5 \mathrm{~cm}$ ) were black-and-white line drawings of familiar objects drawn from the picture set published by Cycowicz, Friedman, Rothstein and Snodgrass (1997) and were presented against a black background. There were 36 "unique" stimuli belonging to 6 different semantic categories (animals, fruits, clothes, body parts, furniture and vehicles), that were presented repeatedly in the three different conditions. Within each category, the 6 most familiar pictures of objects were chosen (based on familiarity scores for 5-6 year-old-children). In all conditions, the pictures were randomly presented in the $2 \times 2$ matrix. All cues as well as the fixation cross were presented in white (font size 36).

\section{Electrophysiological Recording and Analysis}

EEG activity was recorded from 60 scalp locations using tin electrodes located on an elastic cap (EasyCap). Brain Vision Recorder software and the corresponding Brainamp amplifiers were used for signal acquisition (Brain Products $\mathrm{GmbH}$, version 1.10). One electrode in the EasyCap (FPz) was used as a ground, and the left mastoid functioned as the online reference for all electrodes during measurement whereas the right mastoid was included as active electrode. Vertical eye movements were registered with two electrodes positioned above and below the right eye. For measurement of horizontal eye movements two electrodes were placed on the outer canthi of both eyes. Electrode impedance was kept below $10 \mathrm{k} \Omega$, with the exception of the reference and ground electrodes which were held below $5 \mathrm{k} \Omega$. EEG and EOG signals were continuously sampled at $500 \mathrm{~Hz}$ and filtered online with a bandpass filter of 0.05-120 Hz. 
(A)

(B)

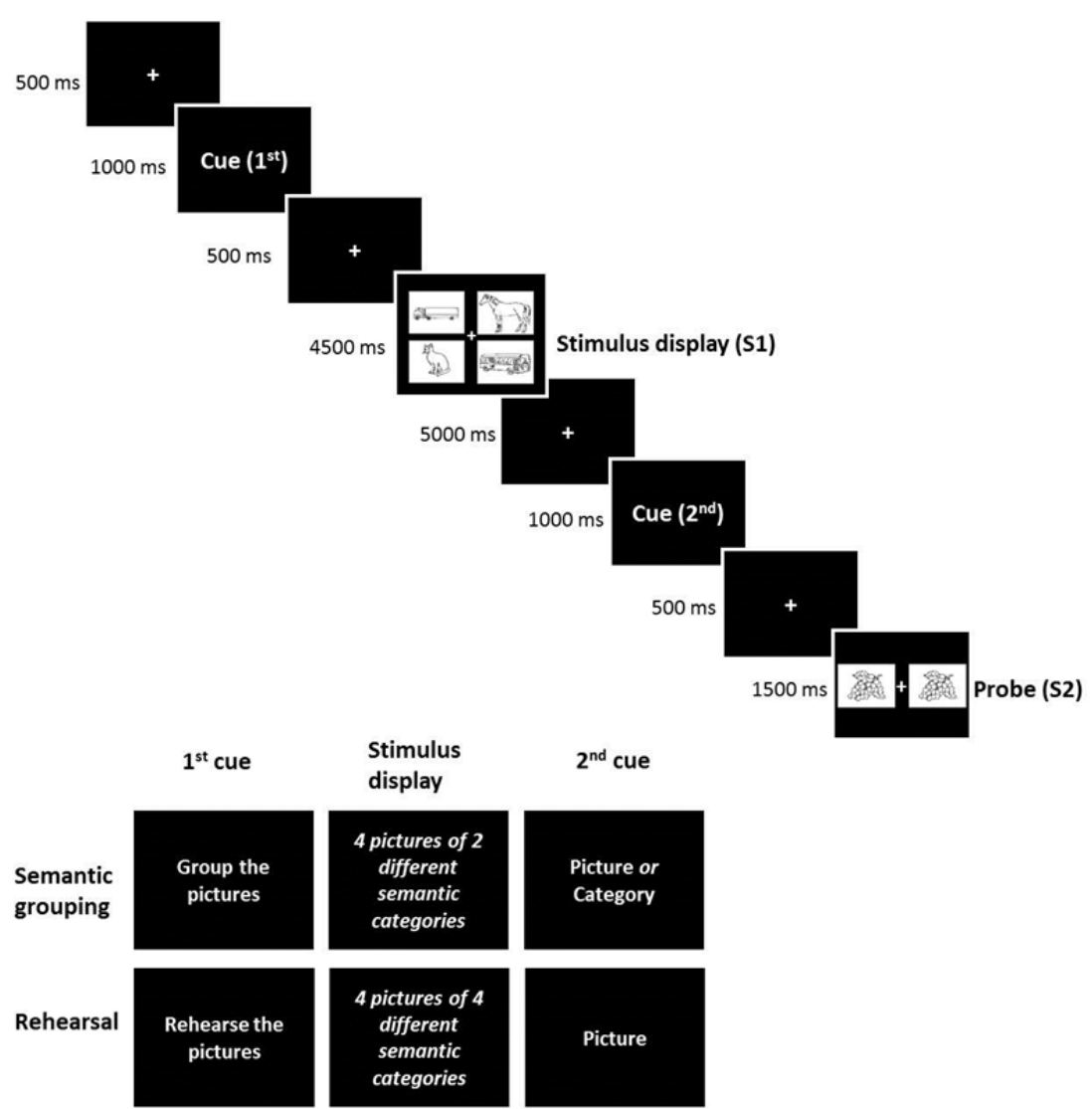

Figure 1. (A) Illustration of the sequence of events in a trial. A first cue indicated the relevant condition for that trial, after which the stimulus display was shown. During the retention interval the memory strategies had to be used in the two experimental conditions, and a second cue then indicated the type of decision that subjects had to make on the following probe stimulus. (B) Schematic illustration of the specific cues and configuration of object pictures in the stimulus display in the two experimental conditions.

ERP analysis was performed using Brain Vision Analyser software (Brain Products $\mathrm{GmbH}$, version 2.0). The continues EEG data were first filtered offline with a lowpass filter of $30 \mathrm{~Hz}$ (24 dB/oct) (encoding-phase) or $7 \mathrm{hz}(24 \mathrm{~dB} /$ oct) (maintenance-phase). Artifacts from horizontal eye movements and blinks were reduced with the algorithm of Gratton, Coles and Donchin (1983) that is available in the Brain Vision Analyser software package. Epochs were made from -200 to 9500 relative to S1 onset, and were baseline corrected using the -200 to 0 pre-stimulus interval. In the next step epochs were created for the encoding (0-1800 ms poststimulus) and maintenance (5000-9500 ms post-stimulus) phase, the latter starting from the time at which the stimuli from the memory set disappeared from the screen. Trials containing EEG artefacts exceeding $\pm 75 \mu \mathrm{V}$ were rejected from the 
data, which was done separately for the $0-1800 \mathrm{~ms}$ epoch and the $5000-9500 \mathrm{~ms}$ epoch. The data were then re-referenced offline to the average of the left and right mastoids. ERP averages were computed separately for each subject in the four stimulus conditions (related and unrelated conditions in both the encoding and maintenance phase). Only trials including correct responses to probe trials were included in the averaging procedure. For encoding, a total of a total of 75 (range 57-102, SD=11.8) and 36.8 (range 24-51, SD=6.6) trials remained for analyses in respectively the semantic grouping and rehearsal condition. For maintenance, 63.9 (range 31-90, SD=16.6) and 31.5 (range 16-47, SD=8.5) trials remained for analyses in the semantic grouping and rehearsal condition, respectively.

\section{Data analysis}

\section{Behavioral data}

Mean reaction times (RT) to correctly identified probes, mean percentage of correctly identified probes ( $\%$ hits) and mean percentage of incorrectly identified probes (\% errors) were computed in semantic grouping-picture and rehearsalpicture trials, separately for yes- and no-response trials. To investigate if memory performance is better in semantic grouping vs. rehearsal trials as we hypothesized in the introduction, we first compared accuracy and RT performance between semantic grouping and rehearsal trials in which subjects had to decide whether the probe picture (S2) was or was not part of the previously presented memory set (S1). Since S1 and S2 stimuli and task demands during the retrieval phase were exactly comparable between both conditions any differences have to be attributed to applying distinct memory strategies. T-tests were applied to reaction time data. Because of a non-normal distribution, accuracy data were log transformed before analyses, and nonparametric Wilcoxon rank sum tests were applied. All analyses were performed separately for yes- and no-response trials. The alpha level for all comparisons was set at $p<.05$.

\section{ERP data}

For analyses of the ERP data we used the BESA Statistics software package (BESA $\mathrm{GmbH}$, Graefelfing, Germany). In BESA statistics, information from all electrodes and all time-points is entered and by means of non-parametric permutation testing the software determines where in time and at which electrode-clusters differences between conditions are statistically significant. The major advantage of this approach is that no a priori selection of electrodes or time windows is required and that only those effects are considered significant that survive correction for multi- 
ple comparisons (thereby decreasing the risk of false positive findings). The procedure that BESA Statistics follows consists of two main steps. In the first step the program performs, in our case, dependent t-tests (Student's t-test; Hays, 1988) to identify statistically significant differences between conditions for every space/time point. The condition differences resulting from this are ordered in so called data clusters. Then, it is determined if these initial data clusters survive permutation testing. To obtain a reliable correction for false positives, 10.000 permutations were performed. The reported data clusters survived a statistical threshold, corrected for multiple comparisons, of $p<.05$. The cluster value represents the sum of all $t$-values of all data points in the respective cluster (see manual BESA Statistics for an elaborate description of the procedure; www.besa.de).

We contrasted semantic grouping with rehearsal trials in BESA Statistics to extract strategy specific spatiotemporal activation. We only report those components identified by BESA Statistics that have in earlier studies been linked to semantic grouping. This was done in order to examine if these ERP components are specific to semantic grouping or if they are also modulated by rehearsal. For encoding we entered the entire window in BESA Statistics (0-1800 ms). With respect to maintenance we were only interested in ERP activity reflecting maintenance of representations held in memory when the sensory input was not present anymore; for that reason only data following S1 offset (from 5000-9500 ms) was entered in BESA Statistics.

\section{Results}

\section{Behavioral data}

See Table 1 for mean RTs, percentage correct responses and errors in the semantic grouping-picture and rehearsal-picture condition (separately for yes and no response trials). While RTs did not significantly differ between semantic groupingpicture and rehearsal-picture conditions for yes-response trials, $t(18)=-1.56$, $p=.14$, for no-response trials subjects responded significantly faster in the semantic grouping-picture condition than in the rehearsal-picture condition, $t(18)=2.73$, $p<.05$. Overall, accuracy levels were quite high and percentages of correct or erroneous responses (in yes- or no- response trials) did not significantly differ between semantic grouping-picture and rehearsal-picture conditions ( $p=.33$ for yesresponse trials and $p=.89$ for no-response trials). Although not depicted in Table 1 , accuracy percentages were also quite high in semantic grouping-category trials ( 90\%), which did not significantly differ from the accuracy percentages in semantic grouping-picture trials ( $p=.75$ for yes-response trials and $p=.83$ for noresponse trials). 
Table 1. Mean reaction times, mean percentage of correctly identified trials (\% Hit) and mean percentage of incorrectly identified trials (\% Errors) in semantic grouping- picture trials and rehearsalpicture trials (separately for yes- and no-response trials). Standard deviations are presented in parentheses.

\begin{tabular}{llll}
\hline Probe Type & Reaction Time $(\mathrm{ms})$ & Hit $(\%)$ & Errors (\%) \\
\hline Yes-response trials & & & \\
Semantic grouping-picture & $977.3(143.3)$ & $88.9(9.4)$ & $4.3(6.3)$ \\
Rehearsal-picture & $959.8(119.6)$ & $91.4(6.4)$ & $4.9(3.9)$ \\
No-response trials & & & \\
Semantic grouping-picture & $930.9(135)$ & $87.9(13.1)$ & $3.7(5.1)$ \\
Rehearsal-picture & $963.9(135.4)$ & $88.3(9)$ & $4.3(6)$ \\
\hline
\end{tabular}

\section{ERP data; Encoding}

BESA Statistics found several significant clusters for semantic grouping vs. rehearsal trials. As mentioned in the introduction, we followed a hypothesis driven approach by only focusing on those ERP components that have previously been associated with semantic grouping in order to examine how possible involved rehearsal processes may account for or influence these effects.

P200. BESA Statistics revealed an increased P200 amplitude in a time window from 164-268 ms post-stimulus for rehearsal vs. semantic grouping trials (cluster value $=-617.993, p<.05)$. As can be seen in Figure 2, this P200 effect was present mainly at right temporal-parietal electrodes.

N400. An increased N400 for rehearsal vs. semantic grouping conditions was found over frontal-central electrodes in a time window from 328 to 440 ms poststimulus (cluster value $=2140.71, p<.01$; see Figure 2).

Late Positive Component (LPC). From 626-660 ms post-stimulus, the semantic grouping condition gave rise to an increased LPC compared to the rehearsal condition over frontal-central electrodes (cluster value $=303.343, p<.05$ ) also see Figure 2.

Sustained positive slow wave. BESA Statistics showed an enhanced sustained positive slow wave in response to rehearsal compared to semantic grouping trials in a time window from 820 to $904 \mathrm{~ms}$ post-stimulus. As can been seen in Figure 2, this effect was distributed mainly over bilateral prefrontal electrodes, but also covered some parietal electrodes (cluster value $=-1464.98, p<.01$ ). 


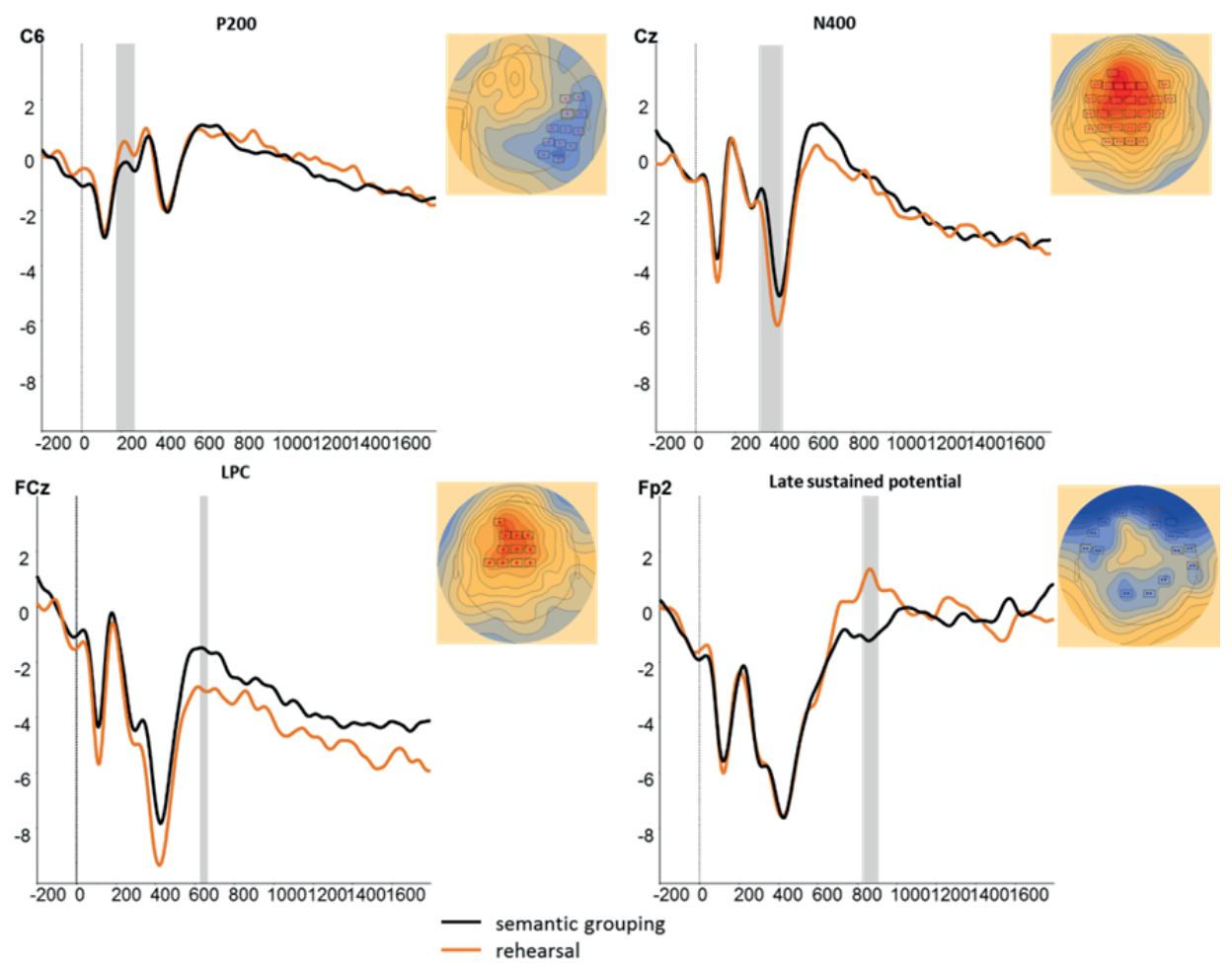

Figure 2. Significant differences identified by BESA Statistics between semantic grouping vs. rehearsal trials for the encoding phase for the P200, N400, LPC and sustained slow wave.

\section{ERP data; Maintenance}

BESA Statistics identified three significant data-clusters in the maintenance interval. These three clusters all represented increased negative slow waves for semantically grouped versus rehearsed information, but in different time windows and (partly) different electrodes. In a time window from 5000-5232 ms post-stimulus this effect was distributed over left frontal and left parietal electrodes (cluster val$u e=-2049.4, p<.05$ ). In another time window (from 5000-6046 ms post-stimulus) this slow wave effect covered right-frontal-temporal electrodes (cluster value=$7540.34, p<.01)$. Finally, somewhat later in time, between 5446 and 7664 ms poststimulus the increased slow wave for semantically grouped vs. rehearsed- trials was observed at left frontal sites (cluster value $=-8470.56, p<.01$ ) (see figure 3). 


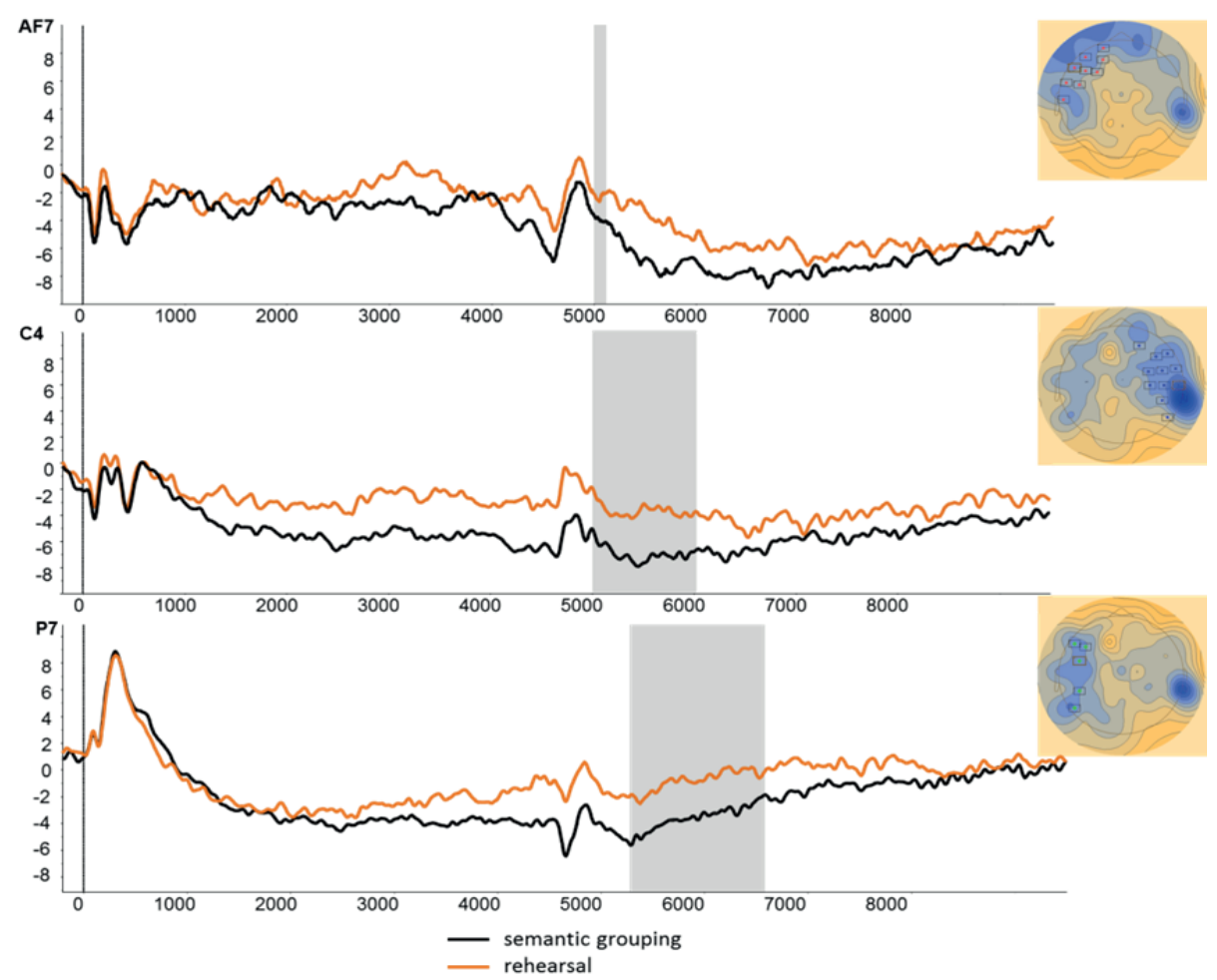

Figure 3. Clusters identified by BESA Statistics for the maintenance phase. The entire 200-9500 ms epoch is shown, but only data starting from $5000 \mathrm{~ms}$ post-stimulus was entered in BESA Statistics to study ERP-maintenance activity when the stimuli were not visible anymore (the peak around $5000 \mathrm{~ms}$ represents the offset- response of S1).

\section{Discussion}

Performing elaborative encoding operations on incoming stimuli increases the likelihood that material can be learned or later remembered. Semantic grouping, which involves rearranging items into similar semantic categories (Mandler, 1967), is a type of elaborative encoding that is central to learning and memory. In the current study we investigated whether ERP components that were earlier shown to be modulated by semantic grouping are indeed specific to semantic grouping, or also (partly) reflect the involvement of simple rehearsal processes that rely on overlapping brain regions as those supporting elaborative encoding (Smith \& Jonides, 1999). To this purpose, during encoding and maintenance phases of a memory task, memory performance and ERP components were compared between trials in which subjects either received explicit semantic grouping or rehearse instructions. 


\section{Behavioral results}

Accuracy was quite high ( $\sim 90 \%)$ and did not differ between semantic grouping and rehearsal conditions. Furthermore, subjects responded faster in semantic grouping vs. rehearsal trials, but only when they had to decide that the probe stimulus was not part of the memory set (no-response trials). There was no difference in RT on yes-response trials. This complies with general findings that encoding material on the basis of semantic features usually leads to better memory performance as opposed to when material is processed superficially during encoding as is the case when simple rehearsal strategies are used (Craick \& Lockhart, 1972). Thus, whereas there was a benefit of semantic grouping in terms of faster recall, this was only present on half of the (no) trials and there were no benefits on accuracy. Such small benefits on recall performance are however not surprising since demands on working memory were deliberately kept low in the present task to be able to study ERP effects specific to semantic grouping and rehearsal without contaminating effects of load. In the following paragraph these ERP results will be discussed.

\section{ERP results: encoding}

The earliest component that showed strategy-effects was the P200, which was increased for rehearsal versus semantic grouping trials over right temporal regions. In a prior study of Blanchet et al. the P200 was found to be increased in a condition requiring subjects to use the semantic grouping strategy (i.e. guided condition). Several factors make it however difficult to reconcile this finding with the current data. First, in the study of Blanchet et al. the P200 was enhanced in guided vs. spontaneous vs. unrelated conditions, but only in the former condition subject received explicit strategy instructions while in this study specific instructions were given in both experimental conditions. Second, the P200 effect observed in the above study had a typical fronto-central distribution (reflecting attentionorienting activity), in contrast to the right temporal P200 topography found in the current study. Mecklinger and Muller (1996) reported on a similar right temporal P200 effect that was greater in conditions that required subjects to encode object stimuli as opposed to conditions in which subjects had to encode spatial stimuli. Further, Moscovitch, Kapur, Kohler and Houle (1995) showed by means of PET that activation in right temporal areas increased when object information was retrieved from LTM (in contrast to retrieving spatial information from LTM). Based on these findings relating right temporal brain activation to object identification processes, we tentatively suggest that the enhanced P200 in rehearsal vs. semantic

grouping conditions might represent more detailed extraction of object identity information in the former, possibly because in these trials all four individual ob- 
jects were analysed. In semantic grouping trials, in contrast, the pictures themselves might have been analysed less thoroughly because they would already undergo more sophisticated processing at a higher level (i.e. semantic grouping).

The P200 effect was followed by a modulation of the N400 component that also was larger in rehearsal compared to semantic grouping trials. This effect is quite surprising in light of studies demonstrating that an N400 appears when information about object categories is retrieved from LTM (Kiefer, 2001, 2005). Although speculatively, the increased N400 in rehearsal vs. semantic grouping trials in the present study could be due to the use of additional strategies besides rehearsal in the former type of trials. Subjects might have attempted to learn the unrelated pictures in an elaborative way by for example forming mental images of them. Such an interpretation would be in line with findings by Kirchhoff and Buckner (2006) who reported that visual (imagery) strategies are among the preferred strategies to remember visually presented object pictures. In addition, a similar N400 effect has been reported in conditions where subjects used visual imagery processes (Nittono, Suehiro, \& Hori, 2002; Riby \& Orme, 2013).

During later phases of the encoding interval, our ERP results did support those reported earlier by Blanchet et al. We also found an enhanced LPC, albeit at partly distinct electrodes as the LPC observed in Blanchet's study, in semantic grouping trials compared to rehearsal trials between 626 and $660 \mathrm{~ms}$ poststimulus. This LPC has earlier been associated with the processing of semantic relations between items, such as deciding whether they belong to the same semantic category (Kiefer, 2001, 2005; Blanchet et al, 2007), which was in the current study required in semantic grouping but not in rehearsal trials in which pictures were semantically unrelated. Furthermore, several other studies reported similarly enhanced amplitudes of a late positive component during encoding especially on trials that were later successfully remembered (DM effect) and this effect (at either frontal or midline sites), was shown to be larger when items were encoded on the basis of semantic associations instead of on physical features of the stimuli (Paller, Kutas, \& Mayes, 1987; Weyerts, Tendolkar, Smid, \& Heinze, 1997). In line with these studies we interpret the enhanced LPC amplitude in the semantic grouping condition as a sign of richer elaboration of the to-be-learned material due to retrieval of category associations.

Finally, against expectations, from 820 to 904 ms post-stimulus, an increased sustained slow wave for the rehearsal vs. semantic grouping contrast was found over mainly bilateral frontal regions. Such increased slow waves have repeatedly been reported in situations in which items are associatively linked to each other in some way (Blanchet et al., 2007; Weyerts et al., 1997). During elaborative encoding WM-load is typically reduced because material is stored in more compact units (Bor, 2003). It may therefore be that in the current study demands on WM were higher in rehearsal vs. semantic grouping trials, because in the former all four indi- 
vidual objects were remembered while in the latter the pictures were organized into two semantic categories. In support of this, Rama et al. (2000) found enhanced amplitudes of late frontal slow waves with increasing memory load, indicating that this slow wave is sensitive to memory-load manipulations. When adopting this WM-load interpretation, the increased sustained frontal slow wave in the rehearsal condition (vs. the semantic grouping condition) might be associated with greater demands on WM-load when encoding unrelated object pictures. But such conclusions need further research in studies incorporating a WM-load manipulation.

\section{ERP results: maintenance}

The hypothesis that negative slow wave components in the maintenance phase of the task would be larger in semantic grouping than rehearsal trials was confirmed by the current data. Such an effect was present at left frontal-parietal (from 5000$5232 \mathrm{~ms}$ post-stimulus), right frontal-temporal (from 5000-6046 ms poststimulus) and left frontal (from 5446-7664 ms post-stimulus) electrodes. Similar increased negative slow waves over frontal regions have repeatedly been found in studies in which participants performed effortful cognitive operations upon to-beremembered information during maintenance (Kiss et al., 2007; Ruchkin et al., 1992). In light of this literature it is suggested that elaborative rehearsal, which involves rehearsing semantically grouped pictures and was required in the semantic grouping condition, consumes more WM-capacity and activation of the prefrontal cortex than simple rote rehearsal required in the rehearsal condition. This interpretation is supported by studies demonstrating that when implementing other types of elaborative encoding strategies, such as chunking material into smaller units or forming mental images of interacting object pairs, activation in prefrontal regions increases (Bor, et al., 2004; Bor, et al., 2003; Bor \& Owen, 2007; Kirchhoff \& Buckner, 2006). The present results add to these prior results by controlling for the impact of simple rehearsal strategies and showing that enhanced prefrontal cortex activation during maintenance of organised material (in this study pictures grouped on semantic category) is specific for elaborative rehearsal strategies.

Although we did not perform source analysis on our data, the increased activation during maintenance of grouped (vs. ungrouped) material above mainly left and right frontal regions is in accord with human lesion and neuroimaging studies showing that the PFC plays an essential role in maintaining information in memory in an organized way (Gershberg \& Shimamura, 1995; Prabhakaran, et al., 2000; Stuss et al., 1994; Wendelken, et al., 2008). We cannot answer the question which specific processes evoked the left and right frontal activation, but some speculations can be made based on results of prior studies. Increased left frontal activation, mostly in Inferior Frontal Gyrus (IFG) or Dorso-lateral Prefrontal cortex (DLPFC), has in several studies been linked specifically to the processing of seman- 
tic features or relations between stimuli during encoding, as opposed to for instance processing of physical relations (Demb et al., 1995; Petersen, Fox, Posner, Mintun, \& Raichle, 1988; Hawco, Armony, \& Lepage, 2013; Innocenti et al., 2010; Savage, et al., 2001). Although the current left frontal cluster was found during maintenance of information grouped on semantic category, it might index IFG or DLPFC activity evoked by the processing/updating of semantic relations between items during maintenance.

The right frontal cortex, in contrast, has been suggested to play a role in the successful application of semantic grouping strategies. Savage et al. (2001) for instance showed that subjects who had the largest recall benefits after using the semantic grouping strategy showed the highest activation in the right frontal cortex. Further, neuroimaging research has demonstrated that activation in the right prefrontal cortex predicts later memory (Brewer, Zhao, Desmond, Glover, \& Gabrieli, 1998; Henson, Rugg, Shallice, Josephs, \& Dolan, 1999; Kirchhoff, Wagner, Maril, \& Stern, 2000; Wagner et al., 1998). Although speculatively, the enhanced right prefrontal cortex activation in related (vs. unrelated) trials in the maintenance phase of the task in the current study could reflect the efficient implementation of elaborative rehearsal strategies. Although the above mentioned studies investigated activity during encoding, because of the limited time resolution of neuroimaging methods these effects might actually (also) represent maintenance-related processes, which might especially be the case when the to-be-encoded stimuli are presented sequentially as is the case in several of the above studies.

Finally, the increased negative slow waves were also distributed above several (left and right) parietal-temporal sites during elaborative vs. rote rehearsal. Various neuroimaging studies have associated activation of parietal-temporal brain regions with memory storage (Coull, Frith, Frackowiak, \& Grasby, 1996; Paulesu, Frith, \& Frackowiak, 1993; Pochon et al., 2001) and the current posterior strategy-effects might hence be indicative of such memory storage processes. Taken together, in agreement with prior studies the present data seem to indicate that a network of prefrontal-parietal brain regions is involved during maintenance of information that is organized or structured (e.g. storage in categories or chunks).

\section{Conclusion}

To the best of our knowledge this is the first ERP study that investigated to what extent the ERP-effects related earlier to semantic grouping are indeed specific to this strategy, or are also modulated by the use of rehearsal. We indeed found that especially during relatively early phases of the encoding interval several ERP components previously linked to grouping material on semantic category during encoding (P200, N400 and late sustained wave) were in fact stronger influenced by processes related to rehearsal in our picture memory task. The fronto-central en- 
coding-LPC and maintenance-related fronto-temporal-parietal ERP slow waves were modulated in the expected direction, i.e. were specifically sensitive to the semantic grouping instruction (and not to rehearsal). The present study highlights the importance of carefully controlling and manipulating the type of strategies used (versus the use of no-instruction control conditions) when examining the neural correlates underlying elaborative encoding strategies. 


\section{References}

Bjorklund, D. F., \& de Marchena, M. R. (1984). Developmental shifts in the basis of organization in memory: The role of associative versus categorical relatedness in children's free recall. Child Development, 55, 952-962.

Bjorklund, D. F., \& Jacobs, J. W. (1985). Associative and categorical processes in children's memory: The role of automaticity in the development of organization in free recall. Journal of Experimental Child Psychology, 39, 599-617.

Blanchet, S., Gagnon, G., \& Bastien, C. (2007). Event-related potential study of dynamic neural mechanisms of semantic organizational strategies in verbal learning. Brain Research, 1170, 59-70.

Bor, D., Cumming, N., Scott, C. E., \& Owen, A. M. (2004). Prefrontal cortical involvement in verbal encoding strategies. The European journal of neuroscience, 19, 3365-3370.

Bor, D., Duncan, J., Wiseman, R. J., \& Owen, A. M. (2003). Encoding strategies dissociate prefrontal activity from working memory demand. Neuron, 37, 361-367.

Bor, D., \& Owen, A. M. (2007). A common prefrontal-parietal network for mnemonic and mathematical recoding strategies within working memory. Cerebral Cortex, 17, 778-786.

Brewer, J. B., Zhao, Z., Desmond, J. E., Glover, G. H., \& Gabrieli, J. D. (1998). Making memories: brain activity that predicts how well visual experience will be remembered. Science, 281, 1185-1187.

Buckner, R. L., Kelley, W. M., \& Petersen, S. E. (1999). Frontal cortex contributes to human memory formation. Nature Neuroscience, 2, 311-314.

Coull, J. T., Frith, C. D., Frackowiak, R. S., \& Grasby, P. M. (1996). A fronto-parietal network for rapid visual information processing: a PET study of sustained attention and working memory. Neuropsychologia, 34, 1085-1095.

Cowan, N. (2001). The magical number 4 in short-term memory: a reconsideration of mental storage capacity. The Behavioral and brain sciences, 24, 87-114; discussion 114-185.

Craik, F. I. M., \& Lockhart, R. S. (1972). Levels of processing: A framework for memory research. Journal of verbal learning and verbal behavior, 11, 671-684.

Cycowicz, Y. M., Friedman, D., Rothstein, M., \& Snodgrass, J. G. (1997). Picture naming by young children: Norms for name agreement, familiarity, and visual complexity. Journal of Experimental Child Psychology, 65, 171-237.

Demb, J. B., Desmond, J. E., Wagner, A. D., Vaidya, C. J., Glover, G. H., \& Gabrieli, J. D. (1995). Semantic encoding and retrieval in the left inferior prefrontal cortex: a functional MRI study of task difficulty and process specificity. The Journal of neuroscience : the official journal of the Society for Neuroscience, 15, 5870-5878.

Fletcher, P. C., Shallice, T., \& Dolan, R. J. (1998). The functional roles of prefrontal cortex in episodic memory. Encoding. Brain : a journal of neurology, 121, 1239-1248.

Gershberg, F. B., \& Shimamura, A. P. (1995). Impaired use of organizational strategies in free recall following frontal lobe damage. Neuropsychologia, 33, 1305-1333.

Hawco, C., Armony, J. L., \& Lepage, M. (2013). Neural activity related to self-initiating elaborative semantic encoding in associative memory. Neuroimage, 67, 273-282.

Henson, R. N., Rugg, M. D., Shallice, T., Josephs, O., \& Dolan, R. J. (1999). Recollection and familiarity in recognition memory: an event-related functional magnetic resonance imaging study. The Journal of neuroscience : the official journal of the Society for Neuroscience, 19, 3962-3972.

Innocenti, I., Giovannelli, F., Cincotta, M., Feurra, M., Polizzotto, N. R., Bianco, G., et al. (2010). Eventrelated rTMS at encoding affects differently deep and shallow memory traces. Neuroimage, 53, 325-330.

Kiefer, M. (2001). Perceptual and semantic sources of category-specific effects: event-related potentials during picture and word categorization. Memory \& Cognition, 29, 100-116. 
Kiefer, M. (2005). Repetition-priming modulates category-related effects on event-related potentials: further evidence for multiple cortical semantic systems. Journal of Cognitive Neuroscience, 17, 199-211.

Kirchhoff, B. A., Anderson, B. A., Barch, D. M., \& Jacoby, L. L. (2012). Cognitive and neural effects of semantic encoding strategy training in older adults. Cerebral Cortex, 22, 788-799.

Kirchhoff, B. A., \& Buckner, R. L. (2006). Functional-anatomic correlates of individual differences in memory. Neuron, 51, 263-274.

Kirchhoff, B. A., Wagner, A. D., Maril, A., \& Stern, C. E. (2000). Prefrontal-temporal circuitry for episodic encoding and subsequent memory. The Journal of neuroscience : the official journal of the Society for Neuroscience, 20, 6173-6180.

Kiss, I., Watter, S., Heisz, J. J., \& Shedden, J. M. (2007). Control processes in verbal working memory: an event-related potential study. Brain Research, 1172, 67-81.

Mandler, G. (1967). Organization and memory In K. W. Spence, Spence, J.T. (Ed.), The psychology of learning and motivation (Vol. 1, pp. 327-372). New York: Academic Press.

Mecklinger, A., \& Muller, N. (1996). Dissociations in the processing of "what" and "where" information in working memory: an event-related potential analysis. Journal of Cognitive Neuroscience, 8, 453473.

Miller, G. A. (1956). The magical number seven plus or minus two: some limits on our capacity for processing information. Psychological Review, 63, 81-97.

Miotto, E. C., Savage, C. R., Evans, J. J., Wilson, B. A., Martins, M. G., Iaki, S., et al. (2006). Bilateral activation of the prefrontal cortex after strategic semantic cognitive training. Human Brain Mapping, 27, 288-295.

Moscovitch, C., Kapur, S., Kohler, S., \& Houle, S. (1995). Distinct neural correlates of visual long-term memory for spatial location and object identity: a positron emission tomography study in humans. Proceedings of the National Academy of Sciences of the United States of America, 92, 37213725.

Nittono, H., Suehiro, M., \& Hori, T. (2002). Word imageability and N400 in an incidental memory paradigm. International journal of psychophysiology : official journal of the International Organization of Psychophysiology, 44, 219-229.

Paller, K. A., Kutas, M., \& Mayes, A. R. (1987). Neural correlates of encoding in an incidental learning paradigm. Electroencephalography and Clinical Neurophysiology, 67, 360-371.

Paulesu, E., Frith, C. D., \& Frackowiak, R. S. (1993). The neural correlates of the verbal component of working memory. Nature, 362, 342-345.

Petersen, S. E., Fox, P. T., Posner, M. I., Mintun, M., \& Raichle, M. E. (1988). Positron emission tomographic studies of the cortical anatomy of single-word processing. Nature, 331, 585-589.

Pochon, J. B., Levy, R., Poline, J. B., Crozier, S., Lehericy, S., Pillon, B., et al. (2001). The role of dorsolateral prefrontal cortex in the preparation of forthcoming actions: an fMRI study. Cerebral Cortex, 11, 260-266.

Prabhakaran, V., Narayanan, K., Zhao, Z., \& Gabrieli, J. D. (2000). Integration of diverse information in working memory within the frontal lobe. Nature Neuroscience, 3, 85-90.

Rama, P., Paavilainen, L., Anourova, I., Alho, K., Reinikainen, K., Sipila, S., et al. (2000). Modulation of slow brain potentials by working memory load in spatial and nonspatial auditory tasks. Neuropsychologia, 38, 913-922.

Riby, L. M., \& Orme, E. (2013). A familiar pattern? Semantic memory contributes to the enhancement of visuo-spatial memories. Brain and Cognition, 81, 215-222.

Ruchkin, D. S., Johnson, R., Grafman, J., Canoune, H., \& Ritter, W. (1992). Distinctions and Similarities among Working-Memory Processes - an Event-Related Potential Study. Cognitive Brain Research, 1, 53-66.

Rypma, B., Berger, J. S., \& D'Esposito, M. (2002). The influence of working-memory demand and subject performance on prefrontal cortical activity. Journal of Cognitive Neuroscience, 14, 721-731. 
Savage, C. R., Deckersbach, T., Heckers, S., Wagner, A. D., Schacter, D. L., Alpert, N. M., et al. (2001). Prefrontal regions supporting spontaneous and directed application of verbal learning strategies: evidence from PET. Brain : a journal of neurology, 124, 219-231.

Schleepen, T. M. J., \& Jonkman, L. M. (2012). Children's use of semantic organizational strategies is mediated by working memory capacity. Cognitive Development, 27, 255-269.

Smith, E. E., \& Jonides, J. (1999). Storage and executive processes in the frontal lobes. Science, 283, 16571661.

Stuss, D. T., Alexander, M. P., Palumbo, C. L., Buckle, L., Sayer, L., \& Poque, J. (1994). Organizational strategies of patients with unilateral or bilateral frontal lobe injury in word list learning tasks. Neuropsychologia, 8, 355-373.

Wagner, A. D., Schacter, D. L., Rotte, M., Koutstaal, W., Maril, A., Dale, A. M., et al. (1998). Building memories: remembering and forgetting of verbal experiences as predicted by brain activity. Science, 281, 1188-1191.

Wendelken, C., Bunge, S. A., \& Carter, C. S. (2008). Maintaining structured information: an investigation into functions of parietal and lateral prefrontal cortices. Neuropsychologia, 46, 665-678.

Weyerts, H., Tendolkar, I., Smid, H. G., \& Heinze, H. J. (1997). ERPs to encoding and recognition in two different inter-item association tasks. Neuroreport, 8, 1583-1588. 
Chapter 6

Summary and Discussion 


\subsection{Summary of the main findings}

Children are constantly presented with new information and skills they have to acquire. For this to be successful it is important that material can be quickly learned and stored permanently in memory. Although learning and memory are important in each phase of our lives, in particular during childhood they are crucial for the learning of new material. This thesis aimed to increase our understanding of the neurocognitive factor(s) that underlie the development of memory (strategies). In Chapter 2 the question was addressed to what extent the development of WM is influenced by increased demands on executive control functions. To investigate this, an N-back task containing three different conditions $(0-, 1-$-, and 2-back) were presented to 6-7, 8-9 and 10-12 year-old children and adults. Correlation analyses between these different conditions and reaction-time interference effects in a flanker task showed that interference control was only required in the 2-back task, and not in the 1-back task. The developmental results showed that performance in the 2-back task (accuracy data) did not reach mature levels before adolescence, whereas 1-back task performance was fully developed at the age of 10-12 years. The study presented in chapter 3 examined if STM or WMC played a role in the development of elaborative strategies, in particular semantic grouping. Children aged 6-7, 8-9 and 10-12 years-old performed a sort-recall task in which they were presented with pictures of objects that belonged to several semantic categories that had to be studied for later recall. To study spontaneous or prompted development of strategy use (semantic grouping), they first performed the task without grouping instructions followed by a version in which they did receive a grouping prompt. Clustering scores served as measures of strategy success. Scores on a forward and backward digit span test were used to index STM-span and WMC, respectively. While 6-7 year-olds did not use the semantic grouping strategy, children aged 8-9 and 10-12 years were able to use this strategy, but the former only after having received the prompt. Both a between-subgroup analysis and a mediation analysis showed that WMC, but not STM, plays an important role in successfully using the semantic grouping strategy in childhood. Chapter 4 described a longitudinal follow-up study of the one presented in chapter 3, which included a subgroup of children of those participating in the cross-sectional study. This study examined 1) through which phases children pass before they spontaneously apply the semantic grouping strategy, 2) if children who recently acquired this strategy are able to transfer it to a new sort-recall task and 3) if WMC was predictive for later semantic grouping strategy use. The results of this study showed that children first are production deficient (i.e. are only able to successfully use a strategy following prompting), before they are able to spontaneously apply the semantic grouping strategy. Further, although production deficient children were equally able as spontaneous strategy users to transfer this strategy to a new task, the for- 
mer were less successful in this compared to the latter. Finally, it was found in a longitudinal design that WMC was not predictive of recall performance in children who successfully used the sorting or clustering retrieval strategy 1.5 years later. The final study included in chapter $\mathbf{5}$ of this thesis aimed to get insight into the neural correlates of the semantic grouping strategy and how they are distinct from those involved in more simple encoding strategies as rote rehearsal. To investigate this, subjects were presented with four related or unrelated object pictures which they respectively had to group on semantic category or rehearse during the encoding period of the task while event-related brain potentials were measured. To also investigate possible differences in ERP activity evoked by maintenance of elaborated (e.g. grouped) versus ungrouped (rehearsed) material, after encoding, subjects were asked to keep the material in memory during a delay interval. At the behavioural level benefits following semantic grouping were only found in terms of faster responding on no-response trials (i.e. deciding that the probe stimulus was not part of the memory set). ERP data showed that during encoding a frontalcentral late positive component (LPC) around $600 \mathrm{~ms}$ was specifically modulated by the semantic grouping instruction. Other earlier components (P200, N400) and a late sustained potential were activated stronger in the rehearsal condition. Confirming fMRI work showing that maintenance of structured information requires more prefrontal cortex activation, during maintenance slow wave activity above left and right frontal-temporal electrodes was enhanced in semantic grouping versus rehearsal.

\subsection{Discussion}

\subsubsection{The role of executive control in the delayed development of WMC}

The findings of chapter 2 suggest that WM and executive control (especially interference control) are closely related to each other since more requirements on executive control deteriorates performance and delays development of WM. Indeed, both constructs not only include similar processes such as selecting relevant above irrelevant information to achieve future goals but also have been shown to rely on overlapping brain regions (Bunge, Ochsner, Desmond, Glover, \& Gabrieli, 2001; Kane \& Engle, 2002). Initial evidence for relations between WM and attention/executive control comes from behavioural studies showing that individuals with high WMC suffer less from distracting information than individuals with low WMC (Kane \& Engle, 2003). Based on these and other findings, in recent years several theories have been proposed that explain how WMC and attentional control are related. An interesting question in this context is how the data of chapter 2 might be explained by or fit within such theoretical models. Two prominent mod- 
els describing how working memory and attention interact are those postulated by Cowan (2005) and Kane and Engle (Engle, Kane, \& Tuholski, 1999). Both models agree in that not working memory itself has limited capacity, rather a capacity limit of (the focus of) attention is thought to constrain working memory span. In other words this means that the capacity of working memory is determined by how well an individual can focus attention on relevant information and task goals. In light of this view it could be argued that, in 2-back conditions involving both interference control and maintenance, children's limited attentional resources have to be shared between selecting the relevant (from the irrelevant) information and keeping in memory the selected 2(back) items. In younger children (i.e. below 12 years) who still have few attentional resources available, attention might have been particularly focused on ignoring the irrelevant letters (i.e. executive control) at the expense of keeping in memory the two target letters. During adolescence, increases in attentional capacity might allow children to allocate sufficient attention to both selection and maintenance of task-relevant information, so that concurrent interference control processes do not deteriorate WM-performance anymore. Although these models can explain why simultaneous interference control processes delay WM development, this has not been investigated empirically. Therefore in future developmental studies the amount of available attentional resources should be manipulated to investigate its impact on executive control/WM development.

As explained in chapter 2, the 2-back task involves the executive processes of both updating and interference control. Although both concepts show a protracted development into adolescence due to their reliance on late developing brain structures within the PFC (i.e. the anterior cingulate cortex and the DLPFC) (Collette \& Van der Linden, 2002; Liotti, Woldorff, Perez, \& Mayberg, 2000; Markela-Lerenc et al., 2004), updating and inhibition (a construct related to interference control) have been shown to be dissociable concepts in both adults (Miyake et al., 2000) and children (St Clair-Thompson \& Gathercole, 2006). This might indicate that updating and interference control contribute differently to the protracted maturation of WM, with one of these constructs delaying WM development more than the other. The current data do not allow to distinguish between these two and this should be topic of future studies.

\subsubsection{The importance of WMC in using the semantic grouping strategy during childhood}

While the cross-sectional study of chapter 3 showed that WMC is an important contributor to concurrent semantic grouping strategy use in childhood, in the longitudinal study described in chapter 4 it was found that WMC was no long-term predictor of semantic strategy use. These seemingly contradicting findings can be 
explained in several ways. First, as mentioned in the discussion of chapter 4, it may indicate that while there are concurrent relations between WMC and semantic grouping strategy use, which is consistent with prior studies in adults (McNamara \& Scott, 2001; Rosen \& Engle, 1997; Turley-Ames \& Whitfield, 2003), WMC is indeed no precursor of later use of this strategy during childhood. Maybe certain cognitive functions developed during the 1,5 year time span between the two measurement points that might have played a more important role in contributing to future use of the strategy, such as increases in a child's semantic memory. The available data do unfortunately not allow us to answer this. Second, the distinct findings of chapter 3 and 4 may actually reflect differences between the specific samples used in these studies. That is, while chapter 3 included children of three different age groups (i.e. 6-7, 8-9 and 10-12 year-olds), in chapter 4 only children of the two younger age groups were included because the 10-12 year-olds left school and were not available anymore to participate in the follow-up study. Given that most 10-12 years-olds probably were spontaneously strategic at time 1 , it is speculated that the WMC-semantic grouping strategy use relation found in chapter 3 was in particular caused by these older children. Also, because WM is better developed in 10-12 year-olds compared to the 6-9 year-olds, it could be argued that WM might not yet act optimally as the mental workspace in which information can be simultaneously hold and processed in younger children (i.e. 6-9 year-olds) and is therefore not predictive of strategy use during early childhood. These reasons, or a combination of them, might explain why in chapter 4 WMC measured at time 1 in 6-9 year-olds was not related to using the semantic grouping strategy 1.5 years later. Possibly, in older children such long-term WMC-semantic grouping strategy use relations do exist. Such hypothesis should be tested in future longitudinal studies that carefully control children's developmental stages.

\subsubsection{How might the observed neural mechanisms (chapter 5) be linked to the developmental pattern of the semantic grouping strategy (examined in chapters 3 and 4)?}

The reason for conducting the ERP-study of chapter 5 was twofold. First, we wanted to get an idea of the brain mechanisms underlying semantic grouping using the ERP technique. Second, the task paradigm in this study was designed as such that it was as similar as possible to the sort-recall tasks used in our developmental memory strategy studies (chapters 3 and 4) so that in possible future studies it might be applied to children to determine the cognitive sub-processes involved during encoding and maintenance of structured information during development. Although it is still speculative at this stage to conclude how the observed neural mechanisms relate to the development of this strategy, several suggestions can be made based on existing studies. 
The encoding-LPC and the negative slow waves prominent mainly above left and right frontal regions were found to be specifically modulated by semantic grouping. The LPC has been suggested to reflect processing semantic relations between items (Blanchet, Gagnon, \& Bastien, 2007; Paller, Kutas, \& Mayes, 1987; Van Petten \& Senkfor, 1996). Several developmental theorists have proposed that increases in a child's knowledge base with age play an important role in the development of semantic grouping strategies (Bjorklund, 1987). In our knowledge base all conceptual and semantic information about items and their relations are stored. Against this background it could be hypothesized that increases in semantic LTM, which enable children to make use of this memory system when grouping to-be-learned material into similar categories, undergoes large development throughout childhood.

Several studies including healthy adults have demonstrated that prefrontalposterior brain regions collaborate in forming new memories, whereby the PFC is thought to select the relevant material which is passed on to more posterior brain regions for storage (Buckner, 1999). In line with this, the current data showed enhanced activity above prefrontal regions during maintenance of grouped (vs. ungrouped) material. Given that in particular prefrontal regions continue to develop into adolescence (Giedd et al., 1999; Gogtay et al., 2004; Sowell, Thompson, \& Toga, 2004), which are thought to serve different functions during semantic grouping, it seems likely that immaturity of the PFC accounts for the late maturational course of semantic grouping strategies described in chapter 3. Although studies investigating the brain mechanisms supporting the development of episodic memory are scarce, this hypothesis seems to be supported by a study by Chiu and coworkers (2006). Here, fMRI was used to compare relations between brain activity during encoding of two different memory tasks and subsequent memory recognition performance in 7-8 and 10-18 year-old children. The first encoding task, verb generation in response to nouns, taxed the process of item-specific encoding. The second encoding task, story listening, included relational or configural encoding, which can be considered as a type of elaborative encoding. To examine which brain regions supported successful episodic memory encoding, the brain activation patterns during both encoding tasks were regressed on their respective memory (accuracy) recognition scores. It was found that activation in the left prefrontal cortex was related to subsequent memory performance in both age groups in the verb generation task and there were no behavioural differences between groups in noun memory performance. For the story comprehension task, left medial temporal lobe (MTL) activation correlated with memory performance in both age groups, but activation in the left anterior MTL and left PFC was related to subsequent memory only in older children who outperformed younger children in this task. Thus, this study shows that when a task poses greater demands on elabora- 
tion strategies, only in older children (who outperform younger children) prefrontal cortex activation is related to subsequent memory performance.

An interesting remaining question is which changes at the brain level or in the communication between brain areas take place during childhood that enable children to successfully use the semantic grouping strategy. Given the findings of chapter 3 and 4 that first signs of successful application and transfer of semantic grouping strategy use is seen around 8-9 years of age (but only after prompting), it seems that the functional architecture of the brain regions supporting such memory strategies is already there by this age, but needs to be refined to function optimally. How might this hypothesis fit within leading theories on functional brain development? Former (maturational) neuropsychological views on functional brain development argued that the emergence of new functions during development is dependent on the maturation of specific brain region(s) (see Mareschal et al., 2007). Because a prominent structure (i.e. the PFC) supporting the use of the semantic grouping strategy has been shown to be one of the slowest regions to mature in terms of structural changes in grey and white matter (i.e. is not fully developed before early adulthood; Giedd, et al., 1999; Gogtay, et al., 2004; Sowell, et al., 2004), this view cannot successfully explain the current finding that 8-9 year-old children already show first indications of successful (semantic) strategy use and transfer, but show ongoing improvement towards early adolescence. These findings seem to be more in line with predictions made by the interactive specialization view on brain development (Johnson \& Munakata, 2005). According to this view the emergence of cognitive functions are not products of the maturation of specific brain regions, but instead are due to changes in the way multiple brain regions interact which is in turn influenced by environmental influences and experience. Because connections between fronto-parietal brain regions (that are involved in the use of elaborative strategies), are still more diffuse and less specialized in children (Durston \& Casey, 2006; Johnson \& Munakata, 2005), it may be that this network is not yet engaged in an optimal way in early childhood, which could explain why children aged 8-9 years need prompting to show (successful) strategy use.

The study in chapter 5 was a first step towards the development of an ERP paradigm suitable for the investigation of the neural correlates underlying strategy use (especially semantic grouping) in children. Future research, including also methods that can say something about the spatial location of involved brain regions (i.e. NIRS), should aim to disentangle the brain regions and their interactions supporting the development of elaborative encoding strategies. 


\subsubsection{Conclusion and practical implications}

The general conclusion emerging from this thesis is that different WM-processes and strategies used to keep material in memory show distinct development patterns, with those depending most on later developing PFC-posterior brain regions, like semantic grouping (see chapter 5), showing a particular prolonged development into early adolescence (chapters, 2, 3 and 4).

Since WM plays a key role in learning, it is also important to consider the practical implications of this thesis. The findings of chapter 2, demonstrating that increased demands on interference control delay the development of WM, imply that learning could be severely impaired when classroom activities place too much demands on executive control and/or working memory. Dividing high-capacity demanding tasks in several smaller parts or providing children with external memory cues (e.g. task instructions written on blackboard) might reduce memory load so that more processing resources are left open to ignore possible distracting information from internal (i.e. inner thoughts) or external sources (i.e. noise in the classroom). Also, minimizing background noise in the classroom, for example by reducing class sizes or letting children wear headphones during execution of WM consuming tasks might be a way to prevent irrelevant information from entering working memory.

In the current study the main focus was on the development (and neurocognitive mechanisms) of the semantic grouping strategy. Needless to say, there are several other memory strategies that can be used during learning, including visual imagery (i.e. creating visual images of the to-be-learned material), chunking (i.e. organizing the material into smaller, more compact units) or the method of loci strategy (i.e. placing items to be remembered along a familiar route) (Bower, 1970; Miller, 1956; Piavio, 1969). In future studies it would be interesting to examine not only the developmental course of these strategies but also their underlying mechanisms. Together with the studies described in chapters 3 and 4 of this thesis this could provide valuable information for education. For instance, if it would appear that certain memory strategies require less WMC for its application than the semantic grouping strategy, then children with poor WM might be encouraged to use those strategies that are within the limits of their WM. By using appropriate strategies children with WM-deficits, who are at risk for developing learning difficulties (Gathercole \& Alloway, 2008), might also be able to perform complex cognitive tasks which allow them to gather the knowledge and skills to make academic progress. 


\section{References}

Bjorklund, D. F. (1987). How age changes in knowledge base contribute to the development of children's memory: An interpretive review Developmental Review, 7, 93-130.

Blanchet, S., Gagnon, G., \& Bastien, C. (2007). Event-related potential study of dynamic neural mechanisms of semantic organizational strategies in verbal learning. Brain Research, 1170, 59-70.

Bower, G. H. (1970). Analysis of a mnemomic device. American Scientist, 58, 469-510.

Bunge, S. A., Ochsner, K. N., Desmond, J. E., Glover, G. H., \& Gabrieli, J. D. (2001). Prefrontal regions involved in keeping information in and out of mind. Brain : a journal of neurology, 124, 20742086.

Chiu, C. Y., Schmithorst, V. J., Brown, R. D., Holland, S. K., \& Dunn, S. (2006). Making memories: a crosssectional investigation of episodic memory encoding in childhood using FMRI. Developmental Neuropsychology, 29, 321-340.

Collette, F., \& Van der Linden, M. (2002). Brain imaging of the central executive component of working memory. Neuroscience and biobehavioral reviews, 26, 105-125.

Cowan, N. (2005). Working memory capacity. Hove, East Sussex, UK: Psychology Press.

Durston, S., \& Casey, B. J. (2006). A shift from diffuse to focal cortical activity with development: the authors' reply. Developmental Science, 9, 18-20.

Engle, R. W., Kane, M. J., \& Tuholski, S. W. (1999). Individual differences in working memory capacity and what they tell us about controlled attention, general fluid intelligence and functions of the prefrontal cortex. In A. Miyake \& P. Shah (Eds.), Models of working memory: Mechanisms of active maintenance and executive control (pp. 102-134). New York: Cambridge University Press.

Gathercole, S. E., \& Alloway, T. P. (2008). Working memory and classroom learning. In S. K. Thurman \& C. A. Fiorello (Eds.), Applied cognitive research in $K-3$ classrooms (pp. 17-40). New York: Routledge/Taylor \& Francis.

Giedd, J. N., Blumenthal, J., Jeffries, N. O., Castellanos, F. X., Liu, H., Zijdenbos, A., et al. (1999). Brain development during childhood and adolescence: a longitudinal MRI study. Nature Neuroscience, 2, 861-863.

Gogtay, N., Herman, D., Ordonez, A., Giedd, J., Hayashi, K., Greenstein, D., et al. (2004). Dynamic mapping of cortical brain development in pediatric bipolar illness. Neuropsychopharmacology : official publication of the American College of Neuropsychopharmacology, 29, S82-S82.

Johnson, M. H. (2001). Functional brain development in humans. Nature Reviews Neuroscience, 2, 475483.

Johnson, M. H., \& Munakata, Y. (2005). Processes of change in brain and cognitive development. Trends in Cognitive Sciences, 9, 152-158.

Kane, M. J., \& Engle, R. W. (2002). The role of prefrontal cortex in working-memory capacity, executive attention, and general fluid intelligence: an individual-differences perspective. Psychonomic Bulletin \& Review, 9, 637-671.

Kane, M. J., \& Engle, R. W. (2003). The contributions of goal neglect, response competition, and task set to Stroop interference. Journal of Experimental Psychology: General, 132, 47-70.

Liotti, M., Woldorff, M. G., Perez, R., \& Mayberg, H. S. (2000). An ERP study of the temporal course of the Stroop color-word interference effect. Neuropsychologia, 38, 701-711.

Mareschal, D., Johnson, M., Sirios, S., Spratling, M., Thomas, M. S. C., \& Westermann, G. (2007). Neuroconstructivism, Volume 1: How the brain constructs cognition. Oxford, England: Oxford University Press.

Markela-Lerenc, J., Ille, N., Kaiser, S., Fiedler, P., Mundt, C., \& Weisbrod, M. (2004). Prefrontal-cingulate activation during executive control: which comes first? Brain research. Cognitive brain research, 18, 278-287.

McNamara, D. S., \& Scott, J. L. (2001). Working memory capacity and strategy use. Memory \& Cognition, 29, 10-17. 
Miller, G. A. (1956). The magical number seven plus or minus two: some limits on our capacity for processing information. Psychological Review, 63, 81-97.

Miyake, A., Friedman, N. P., Emerson, M. J., Witzki, A. H., Howerter, A., \& Wager, T. D. (2000). The unity and diversity of executive functions and their contributions to complex "frontal lobe" tasks: A latent variable analysis. Cognitive Psychology, 41, 49-100.

Paller, K. A., Kutas, M., \& Mayes, A. R. (1987). Neural correlates of encoding in an incidental learning paradigm. Electroencephalography and Clinical Neurophysiology, 67, 360-371.

Piavio, A. (1969). Mental imagery in learning and memory. Psychological Review, 76, 241-263.

Rosen, V. M., \& Engle, R. W. (1997). The role of working memory capacity in retrieval. Journal of Experimental Psychology-General, 126, 211-227.

Sowell, E. R., Thompson, P. M., \& Toga, A. W. (2004). Mapping changes in the human cortex throughout the span of life. The Neuroscientist : a review journal bringing neurobiology, neurology and psychiatry, 10, 372-392.

St Clair-Thompson, H. L., \& Gathercole, S. E. (2006). Executive functions and achievements in school: Shifting, updating, inhibition, and working memory. Quarterly Journal of Experimental Psychology, 59, 745-759.

Turley-Ames, K. J., \& Whitfield, M. M. (2003). Strategy training and working memory task performance. Journal of Memory and Language, 49, 446-468.

Van Petten, C., \& Senkfor, A. J. (1996). Memory for words and novel visual patterns: repetition, recognition, and encoding effects in the event-related brain potential. Psychophysiology, 33, 491506. 


\section{Dankwoord}


Dit proefschrift was er niet geweest zonder de inzet en hulp van anderen. Deze mensen wil ik graag bedanken.

Ten eerste Lisa, mijn copromotor en dagelijks begeleider. Jij hebt een onmisbare bijdrage geleverd aan dit proefschrift. Of ik nu een praktische vraag had over het testen van de kinderen, iets wilde weten over data-analyse, of ergens niet uitkwam met schrijven, jij gaf altijd net die input die me weer op weg hielp. Ik kan oprecht zeggen dat ik veel geleerd heb de afgelopen jaren. Ook dank je wel voor je luisterend oor en de fijne gesprekken over alle andere dingen in het leven. Ik ben erg blij dat ik je ook op die manier heb leren kennen.

Bernadette, mijn promotor. Ook al ben je er pas op het laatst bijgekomen, graag wil ik je bedanken voor je suggesties en aanvullingen op mijn proefschrift.

Dit proefschrift was er niet geweest zonder de deelname van alle scholen en kinderen. In het bijzonder wil ik Basisschool Hulsberg en Basisschool de Schakel in Kerkrade bedanken voor hun enthousiaste deelname aan de verschillende studies.

Mijn collega's van de ontwikkelingspsychologie groep, Hanneke, Linda, Wijnand, Hans en Roefje wil ik bedanken voor hun interesse en alle gezellige groepsuitjes, waar ik veel leuke herinneringen aan heb. Ik ben benieuwd wat er dit jaar op het menu staat bij ons kerst-etentje. Hanneke, bedankt dat je me de kans geeft nog even te blijven in onze groep. Ook wil ik Harry bedanken voor alle leuke (meestal niet werkgelerateerde) gesprekken.

Daarnaast wil ik een aantal "oud-collegas" bedanken. Marjolein, met jou heb ik de langste tijd doorgebracht. Ik heb leuke herinneringen aan alle congressen die we samen hebben bezocht en de tijd die we samen hebben doorgebracht, bijvoorbeeld bij het testen van (de soms bijzondere) proefpersonen of gewoon tijdens een koffie-pauze. Ik hoop niet dat ik je teveel van het werk heb afgehouden met mijn geklets. Datzelfde geldt voor Linda. Bedankt dat je altijd zo behulpzaam bent. Petra, jou ken ik al sinds mijn stage. Ook wij hebben samen veel congressen bezocht samen met Marjolein. Ik heb leuke herinnering hieraan. Ellen, met jou heb ik maar kort een kamer gedeeld, maar wat me daar vooral van bijstaat is dat je altijd enorm geconcentreerd aan het werk was en je niet liet afleiden door andere dingen. Sanne, mijn huidige kamergenoot, bedankt voor de leuke gesprekken.

Alle collega's van CN wil ik bedanken voor de fijne sfeer en leuke uitstapjes. I would like to thank Kamil for arranging the post-doc position.

Ook wil ik graag Annemie bedanken, die voor een groot deel van mijn AIO-tijd het secretariaat beheerde. Dank voor het regelen van van alles en nog wat. Ik vind het fijn dat je je plek nu hebt gevonden. Christl, Riny, en Eva bedankt voor alle regelzaken.

Onmisbaar zijn de stagiaires die hebben meegelopen in de verschillende projecten. Roefje, jij was mijn eerste stagiaire. Bedankt voor je inzet en leuk dat je nu ook in onze groep werkt. Eline en Marleen bedankt voor het verzamelen van de longitudinale data. Eline, Marleen, Saskia, Sandra, Dominique en Marjolein, sta- 
giaires van het ADHD-trainingsproject, dank voor jullie inzet en de fijne samenwerking.

Johan, Erik en Rénee van Instrum wil ik graag bedanken voor alle technische ondersteuning en hulp.

Jenny, ruim 18 jaar geleden bij toen we bij elkaar in de brugklas zaten gingen we niet met elkaar om, totdat we in 4 atheneum opeens naar een andere locatie moesten met onze klas. Dat schiep verbondenheid en sindsdien is de band is altijd goed gebleven. Nu sta je zelfs naast me als paranimf. Ook al betrof ons promotieonderzoek totaal verschillende vakgebieden, ik vond en vind het erg fijn alle (AIO) lief en leed met jou te delen.

Daisy, Marinke en Richard, we zijn samen begonnen aan de studie psychologie in Maastricht en met Daisy en Marinke heb ik uiteindelijk dezelfde master gedaan. Daisy, ook al ben je verhuisd naar Leiden om daar te promoveren, het contact is altijd goed gebleven. Altijd als ik een mailtje van jou krijg is mijn dag weer goed. Marinke, bedankt voor alle gezelligheid, ook samen met Peter en de kinderen. Ik vind het erg leuk om ervaringen uit te wisselen over Samuel en Robin die in dezelfde fases opgroeien. Richard, ook jij bedankt voor de gezelligheid als we elkaar zien, ook samen met Wendy en Mia. Maurizzio wil ik ook graag bedanken voor alle gezelligheid en de hulp.

Ik wil mijn (schoon)familie bedanken voor hun interesse en de afleiding, op welke manier dan ook. Mam, ik vind het fijn dat je me altijd zo vrij hebt gelaten in mijn keuzes. Bedankt dat je zo een lieve oma en moeder bent.

Mijn broertje Roy bedankt voor de gezellige etentjes en andere uitstapjes, ook samen met Nadia (en Jesse natuurlijk) en voor je hulp bij onze verbouwing. Ik vind het fijn te weten dat je altijd voor ons klaarstaat.

Andre, al jaren mijn maatje en levenspartner. Als eerste dank je wel voor het programmeren van de taakjes van een aantal studies. Maar veel belangrijker voor het naar me luisteren, je steun en de afleiding. Ik kan niet in woorden uitdrukken hoe blij ik met je ben, en met onze lieve Samuel natuurlijk. Ik denk dat we trots mogen zijn op alles wat we samen bereikt hebben. Dank je wel voor alles. 
Curriculum Vitae 
Tamara Schleepen was born on the 23rd of October 1983 in Heerlen, the Netherlands. She attended College Rolduc in Kerkrade and obtained her VWO diploma in 2002. In the same year Tamara started studying Psychology at Maastricht University. In 2005 she received her Bachelor's degree in Biological Psychology, followed by a Master's degree in 2006 in Developmental Psychology (Cum Laude). In 2007 and 2008 Tamara worked as a research- and teaching assistant, first under supervision of Leo Blomert and then under supervision of Lisa Jonkman. In 2009 she started as a PhD-student in the Developmental Cognitive Neuroscience section (supervisor: Lisa Jonkman). Tamara currently is a post-doc researcher in the same group under supervision of Hanneke van Mier. 